\title{
Connected at the joints
}

Citation for published version (APA):

Willems, W. L. (2021). Connected at the joints: Calibrating knowledge, morals and health care. [Doctoral Thesis, Maastricht University]. Maastricht University. https://doi.org/10.26481/dis.20210910ww

Document status and date:

Published: 01/01/2021

DOI:

10.26481/dis.20210910ww

Document Version:

Publisher's PDF, also known as Version of record

\section{Please check the document version of this publication:}

- A submitted manuscript is the version of the article upon submission and before peer-review. There can be important differences between the submitted version and the official published version of record.

People interested in the research are advised to contact the author for the final version of the publication, or visit the DOI to the publisher's website.

- The final author version and the galley proof are versions of the publication after peer review.

- The final published version features the final layout of the paper including the volume, issue and page numbers.

Link to publication

\footnotetext{
General rights rights.

- You may freely distribute the URL identifying the publication in the public portal. please follow below link for the End User Agreement:

www.umlib.nl/taverne-license

Take down policy

If you believe that this document breaches copyright please contact us at:

repository@maastrichtuniversity.nl

providing details and we will investigate your claim.
}

Copyright and moral rights for the publications made accessible in the public portal are retained by the authors and/or other copyright owners and it is a condition of accessing publications that users recognise and abide by the legal requirements associated with these

- Users may download and print one copy of any publication from the public portal for the purpose of private study or research.

- You may not further distribute the material or use it for any profit-making activity or commercial gain

If the publication is distributed under the terms of Article $25 \mathrm{fa}$ of the Dutch Copyright Act, indicated by the "Taverne" license above, 



\section{COLOPHON}

ISBN 978-94-6416-729-0 978-94-6416-725-2

NUR 730

Cover design and layout: Auke Wieringa | www.studioburo.nl Printing: Ridderprint | www.ridderprint.nl

(C) Willemine Willems, 2021

The research has been funded by the Academic Collaborative Center on Sustainable Care, which is an initiative of Maastricht University Medical Center+ and Maastricht University.

\section{Sustainable \\ ${ }^{\text {LVING LAB }}$ Care}

The printing of this dissertation has been financially supported by the Netherlands Graduate Research School of Science, Technology and Modern Culture (WTMC). 


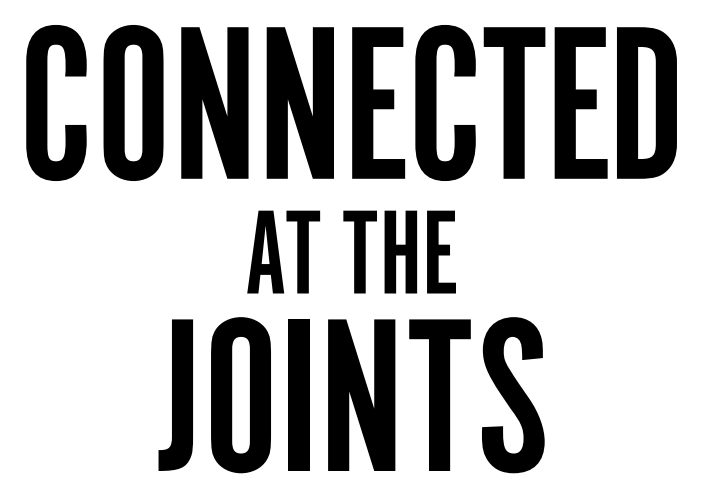

\section{CALIBRATING KNOWLEDGE, MORALS AND HEALTH CARE}

\section{PROEFSCHRIFT}

ter verkrijging van de graad doctor aan de Universiteit Maastricht, op gezag van de Rector Magnificus, Prof. dr. Rianne M. Letschert volgens het besluit van het College van Decanen, in het openbaar te verdedigen op vrijdag 10 september 2021 om 14.00 uur

\section{DOOR




\section{PROMOTORES}

Prof. dr. T.E. Swierstra

Dr. J. Mesman

Prof. dr. P.G.M.C. Vergauwen

\section{BEOORDELINGSCOMMISSIE}

Prof. dr. P.F. Peters (voorzitter)

Prof. dr. M. Boenink, Radboud UMC Nijmegen

Dr. T. Sharon, Radboud UMC Nijmegen

Prof. dr. R. de Wilde

Prof. dr. S. Wyatt 



\section{CONTENTS}

ACKNOWLEDGEMENTS

CHAPTER 1 — INNOVATING HEALTH CARE IN THE MANGLE OF PRACTICE ........................... 11

Evoking responsibility ................................................................................................ 13

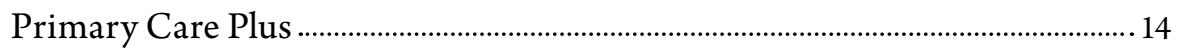

Assessing outcomes in a complex care reality …………………………………………... 16

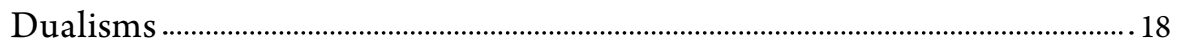

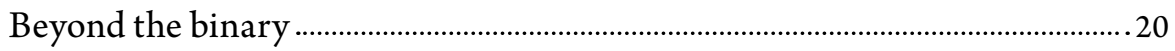

The mangle of practice …………………………………………………………………... 21

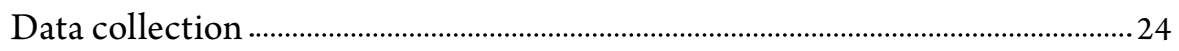

Promises of Primary Care Plus …………………………………………………………. 25

CHAPTER 2 - REPRESENTING THE INNOVATION-REALITY. INTEGRATION,

PATIENT-CENTEREDNESS AND NUDGING ………………................................................... 28

Learning through collaboration .............................................................................. 29

Patient-centeredness …………………………………………………………………. 33

Efficiency through relocation .......................................................................................... 38

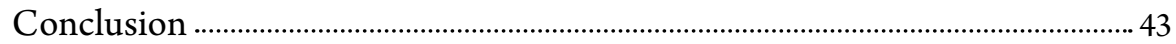

CHAPTER 3 - INTEGRATED-CARE-IN-ACTION. THE KNOWLEDGE WORK OF

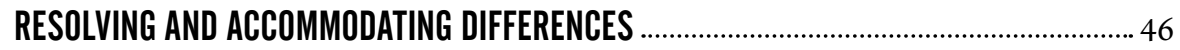

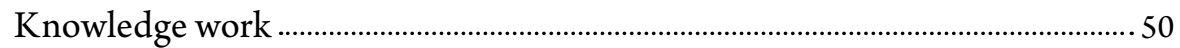

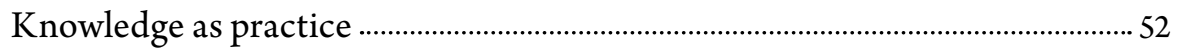

Juggling problem and solution ......................................................................................... 57

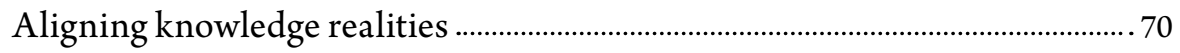

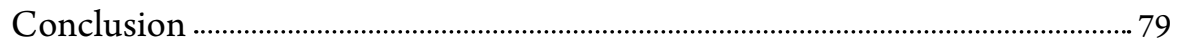

\section{CHAPTER 4 - CRAFTING PATIENT-CENTEREDNESS. THE INTELLIGENT ROUTINES}

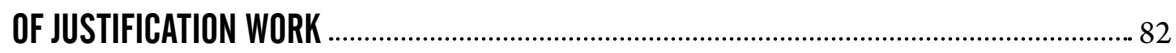

Agency and morality as practice …………………………….................................. 85

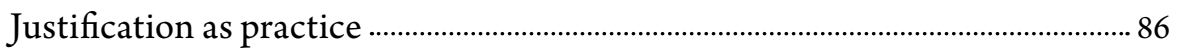

Moral justification work ................................................................................................. 89

Patient-centeredness as a routine shaped over time ………………......................93

Crafting patient satisfaction ............................................................................................. 99 
Navigating the material agency of absent high-tech diagnostics 104

Conclusion

\section{CHAPTER 5 - TURNING OF MINDLESSNESS. HOW TO INTERVENE IN}

Intelligent routines

Situationism mediated by meaning 123

Nudging as an intervention that promotes scaffolding . 126

Reflections on the duality of agency 128

Duality of design and action 130

Legitimacy and nudging 135

CHAPTER 6 — CONNECTED AT THE JOINTS

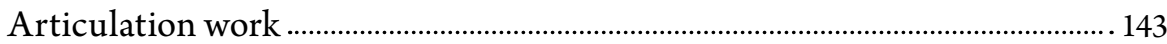

The emerging agencies of Primary Care Plus and beyond ………...................... 147

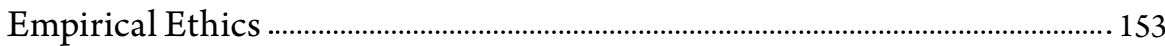

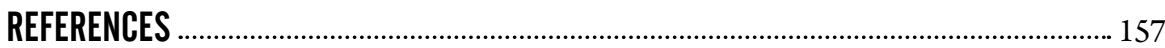

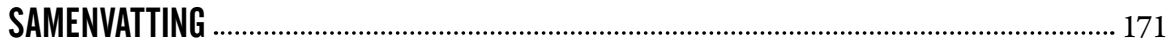

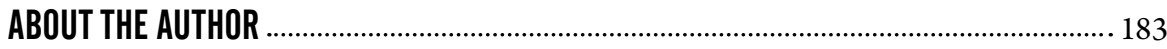




\section{ACKNOWLEDGEMENTS}

When starting the research project in 2014, I left the city and university where I had lived, studied and worked for over 12 years. Moving to the other side of the country in order to conduct a research that entailed combining different faculties, research traditions and methods, it sometimes felt I was living many lives at the same time. Connecting these diverse segments at the joints would not have been possible without the innumerable people who inspired and supported me, and who gave the right words and ideas when I needed them.

I am grateful for being guided by three giants of supervisors, each with a completely different character and skill set, but equally supportive and inspiring. Tsjalling Swierstra kept on challenging me to clarify my ideas and reshape the argumentations that I wanted to convey in my texts. Demanding, but supportive, he made me believe that I had what it takes to finish a dissertation and continue the academic work. Jessica Mesman's passion for meticulously following medical professionals inspired me to keep my ears, eyes and mind open for seeing the rich details of their daily work. Providing me with the right metaphors at the right times she dragged me through the writing process when it was hard. Philip Vergauwen made time whenever called upon to provide critical though encouraging feedback, and to make suggestions for a truly interdisciplinary take on the topic.

I am indebted to the many colleagues and friends who during the years that I worked in Maastricht inspired me with their ideas, their feedback and with whom I had joyful and comforting moments. It would not have been the same without Dani, Claudia, Sarah, Ties, Marith, Linnea, Merel, Tamar, Jacqueline and all the other MUSTS, MEPET and Philosophy colleagues, too many to mention. Daan, Sofie and the other researchers of the Academic Collaborative Center of Sustainable Care, helped me to also feel at home - academically and socially - at the "other side" of the river. A special mention of Floortje - partner in leaving the comforts of our home university for the far-away city of Maastricht - and Gili, always eager to help in whatever way possible. Being part of the EPET research group made me feel academically connected. I am grateful to Karin, Marianne, Simone, Olya, Mira, Mayli, and all the others for joining the quest of figuring out what empirical ethics could or should be. Another important source of inspiration and knowledge were the workshops and summer schools of the WTMC. My compliments to Teun, Willem, Gary, Bernike and Govert for offering creative and challenging ways to engage with our field of STS. 
When the work in Maastricht was over, the dissertation was not. At my new position at the Athena Institute, my colleagues gave me inspiration and reason to finish what sometimes seemed like an impossible job. I am thankful to Anouk, Joyce, Lisa, Aafke, Marjoleine, Jantien, Tessa and Annemarie for showing how inspiring team science can be and to Virgil for being in the same boat and for unconditional support. I am grateful to Frank and Jacqueline for recognizing potential when I was not sure about it myself.

I want to thank Pim and Sadije, two especially strong people, who are willing the stand next to me when I will defend the dissertation, my sister, Harmke, and my friends, Joska, Sara, Elena, Chaja, for always being willing to hear about the joys and hardships of the process, Patricia and Lieke, for cheering me on at the right moments with the right memes and emoticons, and Ester and John for praying for the best outcomes.

The two people at least as involved in the project as me are my parents: baby-sitting, correcting the Dutch summary and impatiently waiting until the work can be celebrated. Nothing has inspired me more than my own mother, who with her idealism and will power managed to make the world a better place for many people, and my father, always being on her side.

Last but surely not least, I want to thank Filipe, Thiago and Oscar, the three special men in my life, for being my frogs whenever I feel like toad. Thanks for reminding me daily that "if a running try", a "running and waving try", and a "running, waving and jumping try" do not work, the "running, waving, jumping and shouting try" will surely be effective. Filipe, you telling me that I just have to keep pushing, made me keep pushing early morning, and late at night. You managed to silence my robins. Let's sit back now and watch the dissertation fly "way up at the top of the sky". 


\section{1 \\ INNOVATING HEALTH CARE IN THE MANGLE OF PRACTICE}

Two months before the Dutch parliamentary elections of 2017, one of the national broadcasting organizations screened a show at prime time called The National Healthcare Debate. That year, media and political parties had designated health care to be the main topic of the national elections, the issue that the voters were expected to be most concerned about. The debate opens with a discussion between three people, a general practitioner, a director of an insurance company and a patient, sitting around a table in the studio. Starting the discussion, the presenter asks the director how the insurance company deals with the inability of a growing group of patients to pay their hospital bills (in time). This immediately gives the director an opportunity to showcase the insurance company's policy of social responsibility: "We really try to help defaulters. For example, by giving them the option to pay in terms." Listening to his response, the general practitioner's face clouds over. She sits up and exclaims: "Defaulters!?... We are talking here about our people, our fellow citizens, our fathers, our mothers!"

The above discussion, unfolding during the National Health Care Debate, gives an insightful impression of how the health care market, ten years after its installation, has become a point of contention. When regulated competition was introduced in Dutch health care in 2006, proponents saw it as a way of containing the untamable growth of the system, criticized for making care delivery slow, inflexible and barely customer friendly. The carefully designed market system of Dutch health care, policy makers promised, would improve itself without much interference of the government (Van Damme, 2012; Enthoven \& Van de Ven, 2007; Van de Ven \& Schut, 2008).

In the Dutch health care system, stakeholders have specific market roles. Insurance companies purchase care from health care providers, such as general practices and hospitals, on behalf of citizens who pay a monthly premium to be covered yearround for health care costs. Dutch citizens are mandated by the government to buy a package from one of the Dutch insurance companies, risking a fine when failing to do so. Once a year, citizens get the opportunity to switch to another insurance company. This is where the health care system resembles a market most; insurance 
companies try to attract new customers by offering lower prices and better services. General practitioners and hospitals are on the supply side of care as well. In their case, the insurance companies are the ones buying the products, while the patients merely 'consume' them. Providers, such as hospitals and general practitioners, do not sell services individually but in bundles, organized through so-called care groups led by health care providers (Mohnen et al., 2017).

According to the general practitioner in the health care debate, however, installing a market structure does not merely create the right incentives in the right places; it also has a formative effect on the social and moral relations of different participants: For the insurance company, patients who have not paid their bills are defaulters, and thus cease to be "people, our fellow citizens, our fathers and mothers". The strong feeling of injustice that she conveys with her tone of voice and gestures is caused not merely by the words that the director of the insurance company chose to use but by the logic that structures the relations that these words are symptomatic of.

This dissertation revolves around a collaboration of four Dutch health care organizations called "Blue Care", initiated out of a similar dissatisfaction with the state of the health care market. In 2012, three of the partners - two regional (South Limburg), a primary care group and a patient organization, and one national partner, a large health care insurance company - initated the collaboration. Their aim was to rearrange health care services by creating interventions in the delivery of health care tailored to the specific health problems of the population of South Limburg. Eventually, the regional academic hospital joined the collaboration as well. In the documents I analyzed (articles that appeared in the media and mission documents) and the interviews I conducted, I found partners now and again presenting their collaboration as a response to the failures of regulated competition. The former chair of the hospital, for example, who was involved in developing the largest project of the pioneer site, Primary Care Plus, recounted one of his conversations with the director of the primary care group in the early years of their collaboration: "I told him [the director], 'ask yourself with every step whether you are doing it for health care in the region or to earn money.' The moment you start a company, (...) you intend to earn money. (...) You need to be careful; the temptation is huge. That is why we should get rid of the health care market. (...) It is nonsense."1 Similarly, the director of the primary care group, who initiated the collaboration, remarked in an interview: "Because of the market mechanism in health care, every one of us functions on their own little island, has production incentives, and thinks about their own wallet. As a consequence, health care costs rocket sky-high (...).”2

1. Interview, March 22, 2016

2. Interview, June 10, 2015 


\section{EVOKING RESPONSIBILITY}

Building on the above line of market critique, the initiators presented their projects "interventions" - as experiments in changing behavior of health care stakeholders through innovating how (health) care is delivered. The collaboration itself and the interventions were launched with a strong message, an "ideology", in the words of the director of the primary care group. The message was conveyed with the name of the pioneer site, Blue Care. Around the start of the project and in its first years, the rationale behind the name and thus its message was often recounted in local and national newspapers, marketing material, and in medical professional journals:

Everybody understands by now that we need soot filters to prevent the ice sheets from further melting. For my eight-year-old daughter, separating plastics, paper and garden waste is absolutely self-evident. We understand that we will have a problem if we go on cutting down the green lungs of the earth; that we should import ecologically sustainable wood. With simple concepts such as green energy, we have managed to put the environment and sustainable use of resources and air quality on the map. Now, it is time to do the same thing with (health) care. The quality of care [in the Netherlands] is excellent, but the costs are irresponsibly high in what I would call "old care". It is not sustainable. We need a change of mentality. We need a transition towards behavior that is comparable to what we have seen in our thinking and acting in relation to the environment. It is time to hold colleagues accountable. It is time to realize that even though the system rewards doing extra treatments, [as a responsible individual/professional] you cannot keep claiming expenses. (Bruijnzeels \& Kommer, 2013, p. 7)

The ideology of Blue Care - "a wink to green energy" - played a crucial role in positioning the pioneer site as an experiment aimed at finding sustainable alternatives to wasteful market care. In the interviews, initiators explained the ideology to have been crucial for convincing partners to join the ranks. Within the frame of "old care", the collaboration of Blue Care partners is odd, they emphasized once and again. Especially taking into account that if its leading intervention - Primary Care Plus, the relocation of specialized consultations to a primary care setting ${ }^{3}$ - is successful, a significant number of patients will have their specialist consultation outside of the hospital. This would thus entail a loss of revenue. The director of

3 One of the most important distinctions in the Dutch health care system is between primary and secondary care. Primary care is directly accessible for patients such as consultations at a general practice, dentist care, physiotherapy, occupational therapy and pharmacy. Specialized care in the hospital and outpatient clinic is categorized as secondary care, which means it is only accessible through a referral from a general practitioner. General practitioners thus function as gate keepers. They aim to keep patients from accessing the more expensive specialist or secondary care unless it is strictly needed. Primary Care Plus is located in the middle. While patients still need a referral from their general practitioner, the setting itself is as limited in diagnostic possibilities as a general practice. 
the primary care group recounted: "The first time we spoke with the chair of the hospital board, he said, 'well, I do not understand. My turnover will become your turnover, my reduction your growth. Why would I cooperate?' "4 After a period of negotiations and small talk at network events, it was eventually the "strong story" of the pioneer site "captured by the name Blue Care", emphasizing the responsibility for keeping health care accessible for future generations, that convinced the hospital director to join in. Taking part in the odd collaboration means believing that "there is only one manger out of which all health care providers eat collectively. Everything that one person gobbles down will not be available anymore for anyone else" (Schulpen et al., 2012, p. 6) and acting accordingly.

The above quotations show how the pioneer site's ideology and market critique fit a broader felt concern among stakeholders in health care that also became visible in the health care debate of the above: How health care is organized has consequences for how we see ourselves and others, what we deem important, and thus for the decisions we end up taking. The critical general practitioner in the health care debate and the initiators of the pioneer site alike warn of the unintended side effects of the health care market. When the system frames patients as defaulting participants in a market transaction, they might cease to be humans; when health care providers are framed as self-interested entrepreneurs, they might end up selling more treatments than strictly needed. With the "Blue Care is like green energy" ideology, the initiators offer an antidote: If we evoke a norm of collective responsibility for keeping health care accessible, instead of a market of self-interested buyers and sellers, the behavior of stakeholders will change accordingly.

\section{PRIMARY CARE PLUS}

In 2013, Blue Care was designated one of the nine pioneer sites in health care by the Ministry of Health, Welfare, and Sports. Adopting the concept of population health management (Drewes et al., 2014; Steenkamer et al., 2016), the nine selected initiatives each have developed their own program of health care interventions aimed at improving patient experience and population health, and reducing health care costs. In health science, this is called "the Triple Aim", the belief that high-value health care can only be achieved when pursuing these three goals simultaneously rather than focusing on them individually (Berwick et al., 2008). As part of the policy, the impacts in terms of the Triple Aims were closely monitored by the National Institute for Public Health and the Environment (RIVM) (Drewes et al., 2014; Ruwaard et al., 2014).

4 Interview, June 10, 2015

5 Interview, June 10, 2015 
Blue Care's interventions, presented in the "ideological" framework of Blue Care is like Green Energy, are diverse. Each was designed departing from the belief that collaboration, rather than competition, is the driving force behind creativity, innovation and efficiency. In the period of the ethnographic research for this dissertation, the partners of the pioneer site had set up a handful of interventions. An example is TIPP, a call center that functions as an intermediary between primary and secondary care. General practitioners send their referral letters to TIPP. The call center operators contact the patients and help them choose a secondary care provider on the basis of their medical problems, their preferences and information about quality of care and waiting times. Another project was called "Pharmacy", in which regional general practitioners, medical specialists and pharmacists work together to develop protocols for preferred medication per disease, taking into account costs and quality of the medication. Other interventions were still in the making at the time of the research, for example, a collaboration with the regional department of GGZ, the national organization for mental health care, aimed at making the system and decision making of mental health care professionals and their clients more sustainable.

Blue Care's first and most promoted intervention, and the setting in which most of the ethnographic research was conducted, is the earlier-mentioned Primary Care Plus project. In 2013, the hospital and the regional primary care group started organizing specialist consultations for patients with low complex medical problems in a primary care setting. The idea is that in Primary Care Plus, medical specialists see patients with medical problems that their general practitioners merely need some specialist advice about. In Primary Care Plus, a specialist sees a patient only once, at most twice. During the consultation, the specialist takes a "quick look" at the medical problem of the patient and subsequently writes a short advisory letter for the general practitioner. This is why the consultations are typified as merely "guidance consultations", implying that the general practitioner will stay in charge of the patient's treatment, i.e. she is not transferred to outpatient care, a form of specialist care provided in the hospital or a private clinic without admitting the patient to overnight stay(s). On the basis of a research conducted earlier (Schulpen, 2003) and a feasibility study conducted during the pilot phase (Van Hoof, Spreeuwenberg, et al., 2016), it was hypothesized that Primary Care Plus medical specialists would refer around $25 \%$ of their patients to the outpatient clinic.

Originally, Primary Care Plus was provided at general practices. During the pilot phase of the project, from April 2013 to January 2014, medical specialists of five medical specialties from the hospital ${ }^{6}$ traveled to one of six selected practices to hold consultation hours with patients once a week or once every two weeks. The

6 Internal medicine, dermatology, cardiology, orthopedics and neurology 
general practitioners of, in total, 17 regional practices were invited to refer suitable patients to see a specialist in one of the six general practices. During their consultations, specialists could not use any of the facilities of the hospital, unless they referred the patient there (Van Hoof, Kroese, et al., 2016).

When the ethnographic research started (February 2015), the concept had changed considerably. The feasibility study (Van Hoof, Kroese, et al., 2016), which closely monitored the intervention, had found that during the pilot phase of the project, some of the participating regional general practitioners were hesitant to refer suitable patients to the selected general practices, because they were "afraid of losing patients when referring (...) to another (...) practice" (Van Hoof, Kroese, et al., 2016, p. 6). Also, for some of the general practitioners hosting the Primary Care Plus consultations, the relationship with the visiting medical specialists became "too friendly" (Van den Bogaart et al., 2019, p. 32). Next to that, they ended up referring patients whom they would have dealt with by themselves before the intervention. On the basis of these findings, the consultations were held in two "neutral" neighborhood care centers from September 2014 onwards. In the West side of the city, the organization rents consultation rooms in an already existing building; in the East side of the city, Primary Care Plus consultations are provided in a building of three stories, built especially for this purpose.

After the pilot phase, some other smaller changes were made as well. The first time I attended a meeting of professionals involved in running and delivering Primary Care Plus, in February 2015, six specialties ${ }^{7}$ were holding Primary Care Plus consultation hours instead of five, which would later be extended to nine. ${ }^{8}$ During the pilot phase at the general practices, specialists had to rely merely on their knowledge and experience. In contrast, the new specialties negotiated to have some basic apparatuses at their disposal. The organization purchased an ultrasound machine for the gynecology and urology consultation hours, a flow meter for urology, an Ear, Nose and Throat (ENT) unit for the ENT doctors and a slit lamp for the ophthalmologist.

\section{ASSESSING OUTCOMES IN A COMPLEX CARE REALITY}

As described above, Primary Care Plus is one of the interventions of the pioneer site Blue Care, part of the pioneer site policy started by the Ministry of Health, Welfare and Sports in 2013. Being part of this policy, a university research team extensively assessed the delivery of Primary Care Plus during the pilot phase of the project (May 2013 to January 2014) and also after the opening of two Primary Care Plus centers

7 Rheumatology, dermatology, internal medicine, ophthalmology, ENT medicine, and neurology

8 Urology, gynecology and pediatrics were added. 
until 2018 (Van den Bogaart et al., 2019). In collaboration with the RIVM, the team of researchers, part of the Living Lab of Sustainable Care, focused on outcomes in terms of population health, quality of care and costs (Van den Bogaart et al., 2019; Van Hoof, Kroese, et al., 2016; Van Hoof, Spreeuwenberg, et al., 2016). Later on, the Quadruple Aim (Bodenheimer \& Sinsky, 2014) was adopted (Van den Bogaart et al., 2019), adding a the fourth dimension of health care provider's satisfaction.

Assessing the project on the basis of outcome measures fits the research context of the Minstry's pioneer site policy. In Dutch, a pioneer site is called a "proeftuin", which literally translates to "experimental garden". As such, the different regions in which the selected pioneer sites operate are seen as laboratories; the interventions as experiments that test the innovations before considering them for implementation in other places. An experiment is commonly understood as a systematic procedure used to test a hypothesis. It is a controlled trial. In this case, the hypothesis to be tested is that relocating medical specialists will result in lower costs, increased population health, and improved patient experience and providers' satisfaction (Quadruple Aim). Effects of the care relocation are measured in three or four categories as outcome measures. In order to make sure the measured effects are a result of the intervention and not of anything else, the outcome measures of the relocation are compared to outcome measures of a control group of patients with similar problems, who had visited a medical specialist in the outpatient clinic of the hospital instead.

Measuring not one outcome but three or four simultaneously is seen as a considerable improvement in comparison to how health care delivery is commonly assessed. Yet, the experimental approach described above has its own limitations. Taking the intervention as a way of testing an hypothesis is only possible when the criteria of success and failure are defined beforehand, i.e. with predefined standards that prescribe which outcomes indicate the intervention to be a success and which a failure. In the process of assessment, the outcome measures thus become the main point of access to knowing and understanding the intervention, turning the intervention itself into a black box. What happens during the consultations, how medical specialists give shape to the new form of care delivery, how they deal with the changed environment, how organizational decisions are made in the light of new and unexpected problems and how general practitioners and patients attribute meaning to the changed aspects of the care delivery, remain invisible.

Relatedly, for an experiment such as the Primary Care Plus intervention to be meaningful, it is crucial that its effects are measured in such a way that it can be easily compared to outcome measures of other instances of specialist care delivery. It is not surprising then that the university research team measured three out of 
four outcome measures with questionnaires. Using questionnaires in this context means, in the first place, collecting the conscious and explicit knowledge and opinions that patients and doctors have about the consultations they received (and their physical health). This is seen as giving a sufficient account of how the intervention has performed. Yet, most people, in this case health care professionals and patients alike, have a certain "practical 'blindness' " (Iedema et al., 2019, p. 18) to what they do, how they do it and why. We are blind to those behaviors that Dewey (1922) calls "habit", as "the acquired predisposition to ways or modes of response (...)" that come with a "special sensitiveness or accessibility to certain classes of stimuli, standing predilections and aversions" (p. 42). Habits are important, because "they are the primary means through which we engage with the world: 'Concrete habits do all the perceiving, recognizing, imaging, recalling, judging, conceiving and reasoning that is done'" (Dewey, 1922, as cited in Iedema et al., 2019, p. 16).

Applying this insight on the assessment of Primary Care Plus, it becomes clear that much of what professionals and patients do when delivering, managing or receiving Primary Care Plus, are acquired predispositions to act in a certain way in a certain situation. When assessing an intervention with mainly questionnaires, such actions will unfold without being noted explicitly. The questionnaires, used to measure three out of four outcomes, typically give insight into people's explicit understanding of their experience but not into those actions and experiences that are implicit. Using standardized questionnaires means foregrounding outcomes such as patient and provider's satisfaction and experienced health, while writing habits, tacit knowledge and implicit experiences out of the evaluation.

\section{DUALISMS}

The above notion of experiment and the focus on outcomes instead of processes as determinants of the intervention's succes are part of an implicit set of philosophical assumptions that can also be recognized in the way in which stories about Blue Care and market care are constructed and narrated. In order to develop an approach that foregrounds the processes of Primary Care Plus instead of the outcomes, I will first, in this section, explicate assumptions of agency, morality and knowledge of the assessment methods described above.

The pioneer site policy or, more locally, Blue Care and its Primary Care Plus interventions were set up to find alternatives for the failing health care market. Market care and Blue Care are examples of different, even conflicting systems to direct stakeholder behavior; yet, the promises, expectations and assessment methods have something more fundamental in common. Both the designers of the market and the initiators of Blue Care describe their systems as tapping into the assumed beliefs, 
attitudes and values of stakeholders. Whereas the market was intended to stimulate stakeholders' attitude of self-interestedness, Blue Care initiators sought to go against this effect by evoking norms of responsibility for the collective good.

The above similarity can be pinned down on the implicit concepts of agency in both market care and Blue Care (ideology and interventions). Both are structured by what Iedema et al. (2019) call the "long-standing dualisms separating people from social structures and cultural systems" (p. 15). The first of these dualisms is between structure and agency. Whether the actions of an agent are seen as the outcome of pursuing her self-interest, the result of societal and/or cultural structures, or a mix between the two, the agent is conceptually separated from the social structures in which she acts. Similarly, regardless of how the relation between the health care system and stakeholder-agents is substantiated in the expectations and criticisms of old and new care, system and agent are seen as two separate entities that affect each other and that can be studied in isolation. In both the design of regulated competition and of Blue Care, the stakeholders are invididuals whose behaviors can be affected by organizing health care as a market, or by continuously appealing to their responsibility for the future of health care.

Another of the fundamental dualisms is the one between humans and nonhumans, and relatedly between mind and body. Agency is reserved for humans, because they do not only have a body but also a mind, in which beliefs, attitudes and values are located. The contents of the mind (beliefs, attitudes and values) are thus conceptually separated from the body and seen as the primary cause of the body's actions. These assumptions about agency are at the basis of both market system and the ideology of Blue Care; both are built on the idea that to effectively change the behavior of stakeholders, their beliefs, attitudes and values should be targeted.

Last but not least is the duality between knowledge and actions, on which the assessment method that I problematized in the previous section is based. Knowledge about the effects of the intervention - in case of the Quadruple Aim the measured health, quality and cost effects - can be collected and aggregated, and is thus seen as a sufficient representation of the intervention-reality. Representation and implementation are separated in this view, the first located in the realm of knowledge and the second in the realm of reality, in which actions take place. To be more specific, the understanding of the experiment as a procedure for testing a hypothesis, as described in the previous section, is a product of this duality as well. In the common-sense view of science, which subscribes to the duality of knowledge and action, an 'experiment' is seen as a controlled procedure employed in order to make reality (realm of actions) confirm or reject a hypothesis (realm of knowledge). Or in Popper's (1959) sense of the word, an experiment is a crucial test that makes 
the reality choose between two theories. In the context of Primary Care Plus, the test, i.e. the intervention, and its measured system effects are used for control, and prediction, evaluations and expectations are based on the measured or predicted effects and as such play a crucial role in mobilizing partners, in improving the intervention and in implementing it as a best practice elsewhere. A similar observation can be made about the use of questionnaires. Within the duality of knowledge and actions, Dewey's notion of habit, a form of knowledge embedded in actions, would be an oxymoron, because action and knowledge are two separated entities.

Viewed within this philosophical context, the assessment method, Blue Care ideology and market care form a somewhat coherent whole. In the previous section, I pointed out how the measuring methods of Triple or Quadruple Aim foreground outcomes and explicit knowledge, while rendering the dynamic characteristics of the intervention and the tacit knowledge and implicit experiences of patients and professionals invisible. In this section, I explained how the foregrounding of the one kind of knowledge at the expense of other kinds is closely related to a more fundamental conceptualization of action and agency shaped by the dualities of structure and agency, humans and non-humans, mind and body and knowledge and actions (reality).

\section{BEYOND THE BINARY}

To enable viewing the subject matter with another set of philosophical assumptions in this dissertation, I will go beyond the above binaries. I will do that by using exnovation $^{9}$ as an alternative approach to understanding and studying Primary Care Plus. Exnovation (De Wilde, 2000; Mesman, 2009) is a process by which the everyday accomplishments and complexity of health care practices are foregrounded to enable reflection and learning (Iedema et al., 2019). As such, it does not build a truer or more objective and neutral representation of the world out there, but it focuses specifically on "what people do here and now" (Iedema et al., 2019, Mesman, \& Carroll, 2013, p. 71). Such a focus contrasts considerably with the methodologies of the Triple or Quadruple Aim, which are not focused on how it works or what is done but on the outcomes of whatever is done. With exnovation, it is possible to foreground what was rendered invisible: the improvisations, surprises, implicit experiences and habits unfolding in practice; it will help open up the black box of the intervention.

In the report that came out in 2019, Primary Care Plus was evaluated as fairly successful (Van den Bogaart et al., 2019). The outcomes were roughly the same for

9 Iedema et al. (2019) explain "the term 'exnovation"' to be a combination of "excavation" and "innovation", because it refers to a process in which researchers look "more closely at what is to identify opportunities for change, rather than looking farther afield for solutions" (p.7). 
Primary Care Plus as for regular care (measured in the control group), except for improvements in terms of costs and providers' satisfaction. Especially on the level of individual patients, Primary Care Plus care is less costly than regular care. The evaluation was a crucial experiment, making it possible to seemingly objectively compare new and regular care, i.e. it offered a yardstick, but it could not give an answer to the question what was done there and then. Through exnovation, i.e. bringing into view what was rendered invisible, the black box of the intervention can be opened. This is crucial for three reasons. First, if the intervention starts underperforming, it is possible to track down what it is caused by. Second, if the delivery and managing practices of Primary Care Plus need to be improved, we know what actions to discourage and which to stimulate. Thirdly, whenever there is a need to implement a similar intervention elsewhere, it is possible to know what aspects to bring along, which to leave behind, and which local conditions helped and which went against the innovation to run smoothly. Bringing into view the practices of how Primary Care Plus was delivered and managed will prove to yield not only an answer to whether the intervention was successful or not but also a broad range of insights in, among other topics, the theoretical and practical aspects of innovating the delivery of health care and of interdisciplinary collaboration.

\section{THE MANGLE OF PRACTICE}

The approach of exnovation is part of the large family of practice approaches and theories that have roots in the work of a variety of philosophers, such as Aristotle, Dewey, Heidegger, Marx and Wittgenstein. Since the late 20th century, such approaches have rapidly gained popularity in the humanities and the social sciences. The practice approach or practice theory is not one unified theory. Its main advocates, however, do agree on a handful of distinguishing features (Gherardi, 2014; Nicolini, 2013; Reckwitz, 2002; Schatzki, 2001a). First and most importantly, taking up a practice approach means bringing the activities conducted to create or reproduce the subject matter to the foreground (Nicolini, 2013). In the case of Primary Care Plus, this means foregrounding the activities that create or reproduce its delivery and management. As a minimal definition, practices are seen as "sets of activities" (Schatzki, 2001b), which are not studied in isolation but as situated in the socio-material context in which they take place. From this follow the second and third distinguishing features; the second being a sensitivity to 'space' as the material and temporal locus, in which a practice is anchored. This sensitivity to materiality is exemplified in the important role attributed to material objects, tools and technologies in many practice studies (Bresnen, 2009; Engeström \& Blackler, 2005). The third is a sensitivity for the thoroughly 'social' character of our practices. The social 
is then not seen as a separate category but as inextricably bound up with all other characteristics of a practice. Thus, for practice studies it is crucial to understand the activities that make up the practice as collectively shared and widely understood and enacted. Gherardi (2014), for example, understands the situatedness of actions as the fact that such actions emerge from "a dynamic of interactions", which results from how the participants in these interactions understand the actions of others and how they perceive and interpret the environment. None of these aspects, actions, or perceptions can be understood as separate from others. Fourthly, most practice theorists acknowledge the critical role bodies have in actions, not as separate entities that execute orders of the mind or respond to cues in the environment but as inextricably bound up with the collective habits and routines of which the activities are part.

At the basis of the contrast between exnovation and an approach such as the Triple or Quadruple Aim lies a fundamentally different conception of agency. Practice approaches aim at overcoming the long-standing dichotomy between structure and agency, that is, the fundamental question whether social scientists should place primacy on the individual agent, his actions, interest and intentions, or on the social structures of society as the determining factor for the actions of individuals. With the practice turn in contemporary theory (Schatzki, 2001a), practice researchers shifted from focusing on agency or structure to both as constituent parts of a practice. That is, practice researchers are interested in the interactions between humans among one another and the interactions they have with the material and social environment of which they are part (Schatzki, 2001a). Nicolini (2013), paraphrasing Reckwitz (2002), calls the practice approach agent "the homo practicus, (...) a carrier of practices, a body/mind who 'carries', but also 'carries out', social practices" (p. 4). With this word choice, he seeks to emphasize a contrast with the homo economicus, a rational being who follows his self-interests, and the homo sociologicus, whose actions stem from internalized norms. Schematically applied to how market care and Blue Care are presented, the contrasts between the different positions become clear. The agent of the health care market, a homo economicus, is first and foremost a self-interested decision maker. Blue Care's citizen-stakeholder presumed in the pioneer site's market material is a homo sociologicus, because she is expected to change behavior as a consequence of internalizing the evoked norms about stakeholders' responsibility for protecting health care's future accessibility. Pushing this schematic understanding of agency a step further, the homo economicus is first and foremost a mind who rationally chooses the options that serve her own interests; the homo sociologicus is either a body that follows internalized social structures (norms) or a mind that follows explicit norms and values. As an alterna- 
tive to these three simplified theoretical positions, the practice theory departs from the agent as a homo practicus, who is both mind and body at the same time, an embodied mind inextricably part of the socio-material context.

The practice understanding of agency thus entails a first step in abolishing the dichotomy between humans on the one hand and their social and material environment on the other. The homo practicus, however, is a notion of agency still reserved for human beings. I draw on Pickering (1995) in order to take a next step. Pickering characterizes his approach of the study of science as adopting a "performative idiom", in contrast to the traditional "representational idiom of thinking about science" $(1995$, p. 5). In the "traditional" idiom, subscribing to the dualism of knowledge and action, science is taken as a body of knowledge and an activity aimed at making this body representative of the world out there. Adapting the idiom to the study of health care interventions, the traditional approach to monitoring such interventions is to create a body of outcome measures representational for how an intervention is running. Health science is then the activity of making such outcome measures more and more representative for the intervention-realities.

Pickering's performative idiom, in contrast, offers another point of departure:

one can start from the idea that the world is filled not, in the first instance with facts and observations, but with agency. The world, I want to say, is continually doing things, things that bear upon us not as observation statements upon disembodied intellects but as forces upon material beings. (Pickering, 1995, p. 6)

In this quote, Pickering goes beyond the practice approach as described above. The affected beings are not merely disembodied minds, such as the homo economicus, norm-following bodies or minds, such as the homo sociologicus, or embodied minds, such as the homo practicus, but they denote anything that is part of this world and thus has a performative effect on other parts of it. Webster (2006), who applies Pickering's performative idiom to the study of medical technologies, emphasizes yet another consequence: Attributing material agency to - in his case - medical technologies means acknowledging these technologies have impacts that are principally unpredictable and relational, i.e. they have have a formative effect on the world because of the relations that emerge with other agencies. Webster (2006) points out that Pickering's agency is "always emergent and depends on the ways in which, in practice, human and material agency are intertwined” (p. 4). Pickering (1995) refers to this as the "mangle of practice": "the evolving field of human and material agencies reciprocally engaged in a play of resistance and accommodation, in which the former seeks to capture the latter" (p. 23). Adapting this to the study 
of a health care intervention such as Primary Care Plus, I take the practices through which care is delivered and managed as playing fields. ${ }^{10}$ Studying them as mangles of practice means foregrounding the ways in which tensions are navigated and harmony is established.

Taking up the above elaborated conceptualization of agency in this dissertation, I study the practice of Blue Care, more specifically its largest and leading intervention, Primary Care Plus, as a mangle of material, human and social agencies. The research conducted for the dissertation was part of the same Living Lab for Sustainable Care as the university research team that monitored the intervention of Primary Care Plus following the principle of Quadruple Aim (Van den Bogaart et al., 2019). As explained above, with the Quadruple Aim approach, the researcher team focused on the outcomes of the innovation; they did not show or analyze what was done there and then. By exnovating, i.e. by dissecting the continuous and dialectic processes of managing and delivering care in order to learn and innovate, I aimed to bring into view the intervention's unnoted activities and to develop novel ways of understanding what happens when innovating a practice. When opening up the everyday practices of the intervention itself, the ongoing efforts of professionals and patients to enable durable and smooth running organizational and delivery processes come into view. From chapter three onwards, I will analyze these activities with the notion of articulation work (Strauss, 1985, 1988), which I will then introduce extensively and adapt for the purpose of this study. In the course of the dissertation, I will answer the question how Primary Care Plus is done as a dynamic and heterogeneous practice of making its delivery and management run smoothly.

\section{DATA COLLECTION}

In order to answer this question, I draw on an ethnographic research conducted on and off from February 2015 until March 2018. In the research, I focused on two levels of the practice of Primary Care Plus: the management level and the level of health care delivery. I opened up the management level in two ways: first, with a document analysis (mission statements, vision papers, minutes of meetings) and secondly, with data collection on the work floor, consisting of 40 hours of observations of meetings and of 11 semi-structured qualitative interviews with managers, directors of collaborating organizations and other initiators, which lasted around

10 Pickering himself uses the term "field of force". This resonates with the criticized tendency also typical for the work of Bruno Latour $(1987 ; 1988)$ to analyze any action as a "constellations of forces" (Swierstra, 1992, p. 28). Besides rendering his view on science needlessly cynical - it suggests that science is merely a matter of survival of the fittest - it also leaves no space for internal motivation or belief. With such a methaphor, any action becomes merely the consequence of an external force, which is a reduction that does not do justice to the complex interplay of internal considerations and external factors that shape our actions. By replacing "field of force" with "playing field" I intend to create space for a more diverse interpretation of how actions unfold. 
70 minutes on average.

I accessed the level of health delivery in two ways: first through semi-structured qualitative interviews with medical specialists who regularly work at the center and in the outpatient clinic of the hospital (eight in total) and regional general practitioners who refer patients to the Primary Care Plus Centers (ten in total). The interviews with medical specialists, on average, took 55 minutes each, the interviews with general practitioners 30 minutes. Secondly, I collected observations on three types of health care delivery locations. When present at these locations, I observed interactions in the waiting room, and during consultations, I talked to receptionists, assistants, patients and doctors. The Primary Care Plus centers were the first type of location. For around 75 hours, I was present at one of the two Primary Care Plus centers, where I followed 14 medical specialists, attending, in total, 40 of their consultations and holding short interviews with 41 patients after their consultation. I spent around 20 hours at the second location type, the outpatient clinics of the hospital, doing observations in the waiting rooms and during consultations (27 in total). The consultations were held by four different medical specialists, who each also hold consultations at the centers. With five of the patients I observed, I conducted a short interview afterwards. Third, I spent around 36 hours at three general practices, where I attended 37 consultations in total. I held short interviews with 20 patients after their consultation.

\section{PROMISES OF PRIMARY CARE PLUS}

Throughout the book, I draw on the collected data to analyze and assess different aspects of the practice of managing and delivering Primary Care Plus against the background of three central promises. In the analyses of the marketing material, assessment reports, newspaper and magazine articles, and in the interviews I conducted with initiators of Blue Care, three expectations about the effects of Primary Care Plus stood out most because of how often they were mentioned and how thoroughly they were explained. First of all, collaborating in the delivery of health care was expected to stimulate general practitioners and medical specialist to learn from one another. Secondly, bringing the specialist consultations into the neighborhoods, close to patients' homes in a more informal and accessible location, was expected to improve the patient's care experience. Third, relocating specialists to a primary care setting, where expensive treatments are less easily accessible, would help prevent unnecessary treatment decisions. One of the reports written by the research team of the Living Lab for Sustainable Care confirms this finding by identifying the same goals for the Primary Care Plus intervention of the pioneer site Blue Care (Van den Bogaart et al., 2019). 
In the second chapter, I describe these expectations in detail and I place them in current trends in health care research. For each of the expectations, I explore marketing material, vision documents and interviews with initiators and others involved in organizing Primary Care Plus, in order to dissect the notions of agency, knowledge and/or morality implied in how professionals involved in the project talk and write about why and how Primary Care Plus will yield cost reduction and improve population health and patient experience. These three promises of Primary Care Plus structure chapter three, four and five.

In the third chapter, I depart from the first promise of Primary Care Plus, the idea that collaboration will stimulate professional learning. The initiators of Blue Care like to present themselves as a club of unlikely partners. As explained above, within "old care", they emphasize, a hospital would consider a Primary Care Plus center offering specialist consultations to be a competitor. In the context of Blue Care, this is not the case. One of the benefits of collaborating vertically, i.e. when primary care and secondary care work together, is that the different health care professionals can learn from each other. As such, Primary Care Plus is an example of Integrated Care, an organizational form designed to integrate the health care trajectories of individual patients, but that also extends and broadens the medical knowledge of involved health care providers who would otherwise remain on their own specialized island. The dissection of the first promise in the second chapter lays the groundwork for the third chapter, in which I analyze what collaboration between different medical professionals entails in the context of the bimonthly users' council meetings of Primary Care Plus.

In the fourth chapter, I depart from the second promise of Primary Care Plus: Relocating specialist care for low complex problems to a neighborhood care setting will improve its patient-friendliness and as such fits the increasing importance of patient-centeredness for the provision of health care. In the course of the chapter, I will present and analyze observations from the fieldwork done at the Primary Care Plus locations. I describe in detail the interactions between doctor and patient unfolding in these consultations, and their reflections before and afterwards. In the course of the chapter, I do not measure the patient-centeredness of the studied consultations, nor do I search for a comprehensive definition; instead, I explore what patient and specialist do when they enact patient-centered care in the Primary Care Plus center.

In the fifth chapter, I will focus on the promise of cost-efficiency through relocation of specialist care. Identified as fitting the rationale of 'nudging', i.e. the intentional modification of choice environments in order to direct people's behavior, I build on the rich practice of the Primary Care Plus consultations. In the course of 
the chapter, I dissect four consultations, three of which were analyzed in the fourth chapter. Tracking what was done there and then gives the needed input to ethically assess how Primary Care Plus as a nudge turns out in the practice of health care delivery. In the course of the chapter, I criticize several aspects of the notion of agency at the basis of nudging theory and I sketch the conditions for Primary Care Plus to be a legitimate intervention in the choice architecture of delivering specialist care.

In the sixth and last chapter, I take stock of the findings, presenting a systematically developed set of concepts that gave insight into how the delivery and management of Primary Care Plus has been done in practice. In the course of the chapter, I further elaborate on these concepts and show what role they can play in tracking how innovative and collaborative health care practices run, how they can be improved and how learning can be induced along the way. 


\section{2}

\section{REPRESENTING THE INNOVATION-REALITY INTEGRATION, PATIENT-CENTEREDNESS AND NUDGING}

At the heart of the pioneer site lies the belief that by evoking an ideology that places collective responsibility for keeping health care accessible center stage, stakeholders will act more responsibly, and, thus, health care as a whole will become more sustainable. The largest and most prominent intervention of Blue Care, Primary Care Plus, was designed and implemented with the same sustainability aim in mind. However, as became clear in the previous chapter, in the intervention itself, the aim assumed a more concrete form; following the Tripe/Quadruple Aim (Berwick et al., 2008; Bodenheimer \& Sinsky, 2014; Van den Bogaart et al., 2019; Van Hoof, Spreeuwenberg, et al., 2016) it became the simultaneous pursuit of cost reduction, increased patient experience and provider's satisfaction, and improved population health.

In the previous chapter, I addressed how the Triple/Quadruple Aim shaped the assessment methods that the university research team adopted. Near the end of the chapter, I also argued that Triple/Quadruple Aim did not only come with methods of monitoring and evaluation; it also shaped the rationale of why and how these aims would be achieved by the concrete project of substituting non-complex, medical specialist care provided in the outpatient clinic of the hospital for Primary Care Plus provided in a primary care, neighborhood location. This resulted in three promises at the core of the intervention. Primary Care Plus was promised to yield improved specialist care through professional learning, patient-centeredness and (cost)-efficiency. Exploring the ways in which these promises were conceptualized and interpreted in presenting, promoting and explaining the intervention, I build a solid basis from which I depart in the next three chapters, in which I conduct exnovation, i.e. I make visible what was done there and then, in the practices of managing and delivering Primary Care Plus. In the course of this chapter, I show how each of these promises fits a specific health care trend, analyze them and answer the question what notions of knowledge, morality and/or agency are implied in promise and trend.

In the course of the chapter, I will address the three promises one by one. I will start with the promise of learning through collaboration: By organizing Primary 
Care Plus together, general practitioners and medical specialists would exchange knowledge, each improving and broadening their medical expertise. The second promise is that relocating to a neighborhood center would result in a more patient-centered specialist care delivery. Third is that the relocation health care costs would be reduced because of the effects of the new choice of environment.

\section{LEARNING THROUGH COLLABORATION}

In the ideology described in the previous chapter, the collective and collaborative character of the pioneer site and its interventions was presented as a unique selling point. Phrases such as "the initiators want to change the "health care landscape' together", "we need to prioritize our collective interests over the interests of our companies", or references to our "collectively supported health care plans" and "one common purpose" were omnipresent. The use of analogies in the marketing material is another example. Tellingly, the analogy with "marriage with children and mortgage" is made to argue that "health care is more sustainable if you integrate the work and share the responsibility" (Bruijnzeels \& Kommer, 2013, p. 6).

In the intervention Primary Care Plus, the promise of improvement through collaboration assumed a more concrete form. In short, in interviews and mission documents, partners and doctors brought up that collaboration between primary and secondary care would induce a learning effect on the side of the general practitioners. ${ }^{11},{ }^{12}$ For example, in an interview published in a national newspaper, one of the involved general practitioners explained that he usually never sees or speaks to a medical specialist about the medical problems of their patients. "The general practitioners learn from each other during these shared consultations [Primary Care Plus]," he was quoted, "and of course from the specialist". The interview was published during the pilot phase, in which it was common for the hosting general practitioners to be present during the consultations of their own patients. When the organization decided to relocate the consultations to the neighborhood centers, the hosting general practitioners were disappointed, because they thought the learning effect would go away. However, it was once and again assured that holding consultations in neighborhood centers was still a significant improvement in comparison to referring a patient to the outpatient clinic of the hospital in regards to the learning effect. When such an argument was made, the hospital was depicted as a huge

11 This first promise, professional learning through collaboration, will be the point of departure of chapter one, in which I will scrutinize in detail how, in practice, involved professionals with different knowledge backgrounds address and deal with knowledge differences.

12 Much less often, it was mentioned that medical specialists could learn from general practitioners. When it was mentioned, it was phrased differently. Instead of solving a knowledge deficiency, collaboration would help the medical specialists understand the "relevancy" of a referral, i.e. they would understand the complex social and emotional reasons for referring a patient to a specialist, while this was not necessary from a medical point of view. 
bureaucratic institution. Waiting lists are long; however, even after the specialist has seen the patient, it will take a considerable amount of time before the general practitioner would eventually get a letter back. On top of that, such letters are often merely a scant and hard-to-follow account of the specialist's findings. After the relocation, the unique selling point of learning through collaboration was held up high by emphasizing that when referring to the Primary Care Plus center, "decision lines are [still] much shorter." The expression "short lines" in the context of Primary Care Plus referred to the fact that general practitioners would receive advice much sooner than if they had sent their patient to the outpatient clinic of the hospital. They would receive it when still remembering their patient's problem. Also, because they collaborated in organizational structures and met in information meetings, it would become easier to reach out to medical specialists in case of questions, because they will be more familiar with each other.

Primary Care Plus is thus seen as a solution to an often-mentioned problem of health care systems: in order to keep patients from having expensive but unnecessary secondary care, in some systems such as the Dutch one, general pracitioners function as gatekeepers. Often, however, they do not have enough knowledge about specific medical areas to properly fulfill such a role (Greenfield et al., 2016). In order to keep patients from getting caught up in unnecessary, long and expensive medical trajectories, gatekeepers need to have sufficient knowledge about a broad range of medical areas. For example, it seemed to be common knowledge among professionals involved in Primary Care Plus that general practitioners have notoriously little knowledge about the locomotor system, because it is an underexposed topic in medical studies. After receiving orthopedic "guidance" through a Primary Care Plus referral, the next time, a general practitioner will know what to do when seeing a patient with a similar medical problem. Also, because general practitioners need knowledge about such a broad range of medical problems, it is impossible to "be up-to-date about the possibilities and impossibilities of new diagnostics and treatments" (Van Rhijn et al., 2013).

The line of argumentation in the promise of learning through collaboration in the specialist care delivery of Primary Care Plus resonates with the current trend of taking integration as a key strategy for improving health care (Greenfield et al., 2016). According to the seminal definition of Kodner and Spreeuwenberg (2002, p. 3), integration is

a coherent set of methods and models on the funding, administrative, organizational, service delivery and clinical levels designed to create connectivity, alignment and collaboration within and between the cure and care sectors. The goal of these 
methods and models is to enhance quality of care and quality of life, consumer satisfaction and system efficiency for patients with complex, long term problems cutting across multiple services, providers and settings. [Where] the result of such multi-pronged efforts to promote integration [lead to] the benefit of patient groups [the outcome can be] called 'Integrated Care'. ${ }^{13}$

Following the seminal definition, Primary Care Plus, a "model of (...) service delivery (...) designed to create connectivity, alignment and collaboration", is thus,a form of Integrated Care, which delivers a service that "cuts across multiple (...) providers" and is aimed at improving "quality of care", "consumer satisfaction" and "system efficiency". That such an intervention also has potential to yield a learning effect is in line with other literature in the field of Integrated Care that addresses its knowledge dimensions. Integrated Care is then seen as a model of care delivery that, as aim or effect, improves the knowledge of its participants. Researchers believe that by collaborating across professional boundaries, the involved medical specialists, general practitioners and/or nurses increase their knowledge about specific medical areas. The benefit is described as a research finding ${ }^{14}$ or it is reported to be an important motivation for professionals to collaborate in Integrated Care projects (Berendsen et al. , 2007). In a similar vein, in the articles published about Primary Care Plus by the university research team monitoring the intervention, Primary Care Plus is described as a model of care delivery designed, among other aims, to embed "specialist knowledge into primary care" as a way to "counter the strong existing fragmentation of primary care and outpatient care" (Van Hoof, Spreeuwenberg, et al., 2016, p. 1). In the same article, the hypothesis is formulated that with the advice that the medical specialist sends to the general practitioner after the Primary Care Plus consultation, she "will be better able to determine whether a referral to outpatient care is necessary [in the future]" (van Hoof, Spreeuwenberg, et al., 2016, p. 2).

When viewing the above claims through the lens of Integrated Care, a specific understanding of knowledge and of agency, akin to what Pickering (1995) calls the "representational idiom of thinking about science" (1995, p. 5), emerges. As

13 Kodner and Spreeuwenberg's (2002) definition of Integrated Care only entails the care for patients with "complex and longterm problems". However, the meaning of the term has slightly changed in the past 18 years. In author instructions of the Journal of Integrated Care, for example, the editorial board uses Kodner and Spreeuwenberg's definition without the clause "with complex and long term problems" to describe what kinds of research they are interested in publishing (https://www.ijic.org/about/). This adaption of the definition is a clear indication that the meaning of the term Integrated Care has been broadened in the past 18 years.

14 For example, participants' knowledge was found to be increased in projects aimed at integrating care for diabetes patients (Donohoe et al., 2000; Gucciardi et al., 2016), heart patients (Wiles, 1997), palliative oncology patients (Kaasa et al., 2018), dementia patients (Kümpers et al., 2006), patients with cerebral palsy (Katz, 2012), patients with obesity (Osmundsen et al., 2019), spinal cord injury during pregnancy (Bertschy et al., 2016) and osteoporosis (Gaboury et al., 2013). 
explained in the introduction, with such an idiom, science is taken as a body of knowledge and an activity aimed at making this body representational of the world out there. Adapted to the context of the first promise of Primary Care Plus, the understanding of knowledge in Integrated Care literature, and in the promise of learning through collaboration, medical knowledge is taken to be a body of knowledge representative of the human body, its bodily processes and its interactions with the environment in the world out there. With the continuing specialization of medicine (Bontemps-Hommen et al., 2018; Vosman et al., 2016), in which "specialties are becoming subspecialized, and subspecialties are developing sub-subspecialties" (Cooke, 2013, p. 202), the body of medical knowledge has become increasingly fragmented. Medical science is then seen as problematic, because there is not one body of knowledge but plural ones, each representing another part of the human body, or another type of process in a specific part that are often incommensurable (Mol, 2003). When care integration pertains to knowledge, it refers to the activity of making this currently fragmented body of knowledge into a coherent whole.

When speaking, writing and thinking about medical knowledge in the representational idiom, the doctors as agents become first and foremost possessors and users of knowledge. This is similar to what Gherardi (2000) refers to as the "knowledge as commodity" view ${ }^{15}$, in which knowledge can unproblematically go from one professional to the other by means of collaboration. As Gherardi (2000) puts it, with such a view, knowledge is taken to be something like labor or capital, a product that can be transferred without transforming it. Connecting professionals through health care integration is then seen as a way to enable knowledge to travel from the one to the other professional.

The commodity view of knowledge, but also the representational idiom in conceptualizing science, are both products of the deeply ingrained duality between knowledge and action. As explained in the previous chapter, this dualism entails the assumption that knowledge can be conceptually separated from the actions it represents. Knowledge in these views is reified: It is an entity that exists prior to and independently of processes of appropriation and transferal. In the case of Integrated Care, this implies that an unproblematic uptake and extension of the involved professionals' knowledge can be assumed, while the practices of Integrated Care can be viewed as separated from it. In the case of the first promise of Primary Care Plus, collaboration is the set of actions taken together to deliver and manage Primary Care Plus; the increasing knowledge of involved professionals is an effect that is

15 Slightly less static is the mentalist view of knowledge, which Gherardi describes as assuming that knowledge resides in the mind and that it can thus "be appropriated, transmitted and stored by means of mental processes" (2000, p. 212), such as following a training program or learning new skills through collaborating with others. 
separate from the set of actions. In the context of Primary Care Plus, such a notion of knowledge hides from view how collaboration among professionals with knowledge differences is done.

\section{PATIENT-CENTEREDNESS}

In the opening scene of a promotion video released shortly after the center started operating, Mrs. Van der Pijl is filmed while sitting in one of the consultation rooms. Enthusiastically, she says: "You just jump on your bike and ride into the waiting room. It is a bit more village-like here. For me, that is a huge advantage." With such an opening, a clear attempt is undertaken to capture the second promise that I will discuss in this chapter: Primary Care Plus offers a more patient friendly way of delivering specialist care. The organization of Primary Care Plus often presents itself as bringing specialist care to the neighborhood. Emphasizing the "neighborhood-character" does not merely entail the actual relocation from a location suitable for a hospital serving the entire region to a location close to the patient's doorstep; it also entails the accessible and friendly atmosphere of the center and attitude of the Primary Care Plus specialists. "Dermatologist Herm Martens prefers not to wear his white coat during his Primary Care Plus consultation hours (...). He does not have medical apparatuses at his disposal, and he presents himself rather as a medical advisor than a medical doctor," is how a national newspaper sketches the informal atmosphere at the center. In other sources, the fact that there are no waiting lists and that for the insurance company the consultation counts as a free general practice consultation is often mentioned.

With the second promise, Primary Care Plus fits in the current trend in health care research and innovation to put the patient and her preferences center stage. The term patient-centeredness was first introduced more than 50 years ago as a "way of thinking" in medicine in which a clinician not only diagnoses the illness but also examines "the whole person in order to form (...) an overall diagnosis" (Balint, 1969 , p. 269). With hindsight, those first mentions of the term can be seen as evidence of foresight, fitting a "move" that was gaining momentum later in the century, "from a dominant paternalistic model of communication towards a pluralistic model, which attempts to democratize decision making, share understanding and empower individuals" (Taylor, 2009, p. 150).

Loosely defined as an alternative way of thinking in medicine, it has now become a "core value" (Hudon et al., 2011, p. 155) and a crucial element of health care quality (Epstein \& Street, 2011; Kreindler, 2013; Langberg et al., 2019). Nevertheless, definitions are still rather broad and open. An example is the following definition from a guide to improving patient-centeredness of care (Kreindler, 2013): 
Patient-centered care is care organized around the patient. It is a model in which providers partner with patients and families to identify and satisfy the full range of patient needs and preferences (Frampton et al., 2008, p. 4).

The conceptual elusiveness of the term has become a point of critique for health scientists in the field who have set out to find the right definition as a condition for finding the right methods of measuring and implementing it. It has thus become commonplace to remark that there is not sufficient clarity about what it entails (Castro et al., 2016; Håkansson et al., 2019; Hudon et al., 2011; Ishikawa et al., 2013; Langberg et al., 2019; Mead \& Bower, 2000; Taylor, 2009), or how it should be implemented and measured (Funnell, 2016). Such critical observations are often followed by the recommendation to do more research or by a proposal for another new and more comprehensive definition.

In a systematic review of all articles containing definitions of patient-centered care published between 2000 and 2017, Langberg et al. (2019) found that the influential five dimensions of patient-centered care formulated by Mead and Bower (2000) 19 years ago still cover most of the definitions found in literature. Mead and Bower consider patient-centered care to comprise "sharing power and responsibility", i.e. encouraging the patient to participate in the decision making, building a "therapeutic alliance" between doctor and patient, taking the uniqueness of a "patient as person" and her perspective into consideration, adopting a "biopsychosocial" approach encompassing all domains of medicine, and considering the "doctor as person" whose "values and experiences shape how they practice medicine" (Langberg et al., 2019, pp. 1231-1233). A newly added dimension since the 2000s is what Langberg et al. (2019, p. 1232) call "coordinated care". This dimension comprises concerns over receiving care that is timely and continuous.

Taking Langberg's five dimensions of patient-centered care, it becomes clear that even though not always literally mentioned in the documents studied during the document analysis, or during interviews with initiators, the writing and speaking about Primary Care Plus is pervaded with notions of patient-centeredness. According to an article of the research team that monitored the intervention, for example, one of the three hypotheses of Primary Care Plus is that it will

lead to improved patient experience of care, because patients can receive their care close to where they live, as well as in a timely and patient-centered way due to the GP's coordinating role as a gatekeeper of the healthcare system. (Van Hoof, Spreeuwenberg, et al., 2016, p. 2) 
Another example is the often-used slogan in the context of Primary Care Plus, "suitable care on the right location, by the right person for the right price", which is a clear reference to the latest developments in patient-centered care research, in which the dimension of "coordinated care" has become an important issue of concern.

The quest of finding the essence of patient-centered care, the attempts to construct a true definition, to develop neutral and objective tools to measure it (Hughes et al., 2008), and the common aim of identifying barriers and facilitators (Luxford, et al., 2011; Moore et al., 2017), show how the field of patient-centered care is set in the representational idiom (Pickering, 1995). It assumes that the action conducted in the realm of action can be objectively researched with definitions and dimensions of what it entails in the realm of knowledge. Having a correct representation of what it actually is enables implementation, but it also enables identifying barriers and facilitators external to the patient-centered care itself. Adapting Pickering's representational idiom to the context of patient-centered care, health sciences specialized in patient-centered care make up a body of knowledge aiming to represent what patient-centeredness truly is, what barriers in the realm of action should be taken away, what facilitators should be created. Health sciences is then the activity of gaining more and more knowledge correctly representing the essence of patient-centeredness and the actions needed in the realm of actions to enable its implementation. Schematically understanding the dimensions of patient and doctor as persons within this idiom renders the patient and the doctor into vats of stable and clearly defined preferences and convictions.

The assessment methods by which the patient experiences were measured in the context of Primary Care Plus were also set in a representational idiom. To increase patient experience was one of the Quadruple Aims. The university research team assigned to monitor and evaluate the intervention used items of the Consumer Quality Index (CQ index) (De Boer et al., 2010; Donselaar et al., 2009) to measure how Primary Care Plus patients had experienced their Primary Care Plus consultation (Van den Bogaart et al., 2019). The index items were incorporated in the extensive questionnaire that a group of Primary Care Plus patients (intervention group) and a group of selected patients with similar problems who had been sent to a medical specialist in the hospital (control group) were invited to fill in. The CQ index is a standardized and validated instrument developed to measure quality of care as experienced by consumers, composed as a combination of the CAHPS (Consumers Assessment of Health care Providers and Systems) ${ }^{16}$ developed in

16 The report introducing the CQ index refers to Cleary \& Edgman-Levitan (1997), Darby et al., (2006), Zaslavsky, Zaborski, Ling, et al. (2001) and Zaslavsky, Zaborski, \& Cleary (2002) as sources for the CAPHS system. 
the US, and the QUOTE (QUality Of care Through the patients' Eyes) ${ }^{17}$, a measuring instrument developed for the Dutch health care context. Both the CAHPS and QUOTE "put the experience of the consumers center stage", according to the report by which the CQ index was presented (Donselaar et al., 2009, p. 11).

The motivation for developing such a systematic measuring instrument is to improve the demand-driven approach of the Dutch health care system. Measuring and publishing the patients' experience of health care quality of providers would help health care insurance companies and care consumers to choose between the different options, providers to improve the quality of the care they provide, and the government to monitor the health care system on a macro level (Donselaar et al., 2009). In the context of Primary Care Plus, the research team used the CQ index to enable comparison between control group and intervention group but also to compare its outcomes with other substitution projects of the pioneer site policy that they monitored in the same period.

The final report forms a concluding representation of the patient-centeredness of the three substitution projects that the university research team had monitored (Van den Bogaart et al., 2019). The quality of care as experienced by patients is presented in a bar chart consisting of five bars, each expressing one of the following statements and to what extent the patients in both control and intervention group agreed with it: "the decision was made together", "I have been informed about the treatment options", "the medical specialist informed me in a clear and understandable way", "the medical specialist took my symptoms seriously" and "the medical specialist took enough time" (Van den Bogaart et al., 2019, p. 50). As a representation of Primary Care Plus' patient-centeredness, these categories structure and stabilize how much patient-centeredness was achieved by the intervention reality of Primary Care Plus.

In the first and the second promise of Primary Care Plus, both phrased in the representational idiom, an understanding of knowledge presuming a separation between the realm of knowledge and the world of actions can be discerned. An important difference, however, is that in the first promise, learning through collaboration, it was the aim of the organization itself to contribute to making medical knowledge more representational, i.e. to integrate it, to reduce fragmentation. In the context of the second promise, the aim of Primary Care Plus itself is not to contribute to knowledge about patient-centered care or to make such knowledge more representational but to make care itself, conducted in the realm of action, more patient-centered. The focus is thus not on adding to and improving a body

17 The QUOTE method sources that the authors introduce are Campen, Sixma, Kerssens, \& Peters (1998), Campen, Sixma, Kerssens, Peters, \& Rasker (1997), and Sixma et al. (1998). 
of knowledge but intervening in and improving reality. While Pickering (1995) used the representational idiom to characterize how science is traditionally being conceptualized, the move away from improving knowledge about reality, to improving reality itself, implies a further extension of the idiom to make it applicable to morality as well.

In the rationale behind the promise of patient-centeredness and in the ways it was monitored and assessed, a certain notion of morality was implied. The promise of patient-centeredness is aimed at improving the state of affairs in the realm of reality, following predetermined criteria. These criteria function as a yardstick, indicating when a certain aspect is sufficiently patient-centered, or can be used in the case of Primary Care Plus for translating practices to a uniform system of signs that enable comparison between different health care practices. In such a measurement practice, patient-centeredness is not a body of knowledge that represents the world but an "ethical good", a set of moral norms that prescribe how patient-centered care should be done. The activity of measuring patient-centeredness is then a necessary condition to assess the reality, to determine whether it can be judged sufficiently patient-centered, and — when needed — to improve it.

In this section of the chapter, I have argued that the second promise of Primary Care Plus, improving patient experience by relocating the medical specialist consultations to a neighborhood location, fits the current move towards patient-centered health care. Through the course of the section, I showed how the literature on patient-centeredness gives the impression of a quest for the essence of what patient-centeredness is or should entail, and as such is phrased in the representational idiom. However, placed in the context of delivering and assessing Primary Care Plus, it becomes clear that it implies not only a notion of knowledge as representation but also a notion of morality as representation. With such a notion of morality, good care, in this case patient-centered care, becomes a measurable quality, an ethical good that a practice does or does not contain sufficiently. A predefined set of criteria functions as a yardstick to determine whether a state of affairs in reality is sufficiently patient-centered as defined in the realm of knowledge, or as a translation tool that makes comparison between the messy realities of different instances of providing health care possible. Improving care is then the activity of intervening in reality in order to make it comply more and more to a predefined set of criteria. The predefined set of criteria, both the validated and standardized set, but also loosely defined criteria, such as friendly doctors not wearing a white coat and friendly secretaries, in the second promise of Primary Care Plus, are moral norms (in the realm of morality) prescribing what good care should entail in the world out there. This is thus a representational notion of morality, akin to the representational 
notion of knowledge explained above. In the case of morality, however, it is not the actual world that is represented but an ideal one in which care would truly be patient-centered.

\section{EFFICIENCY THROUGH RELOCATION}

The third and last promise discussed in this chapter is that the relocation of specialist care to a neighborhood care center will make the delivery of health care more cost-efficient. The rationale behind this promise is not only that a primary care setting is a cheaper environment than the hospital with its huge overhead costs, but also because holding a consultation in a low-facility environment has a direct effect on the expectations of patients and the habitual ways of ordering diagnostic tests that specialists supposedly have. Director during a meeting: "A patient who enters the hospital, who has an appointment at the outpatient clinic, sees the specialist in his white coat and she sees this big white building. She will expect (...) a treatment with all the trimmings." 18 For specialists, it is the "hospital reflex", as one of the managers calls it in an interview, that causes unnecessary costs. Specialists in a secondary care setting "feel at ease to order all kinds of [unnecessary] tests"19. The relocation will thus lead to fewer expensive treatments that are redundant from a medical point of view.

Even though initiators, managers or others involved in the project do not explicitly call it a form of nudging, the logic of the promise of increased cost-efficiency fits the nudging model: the idea that changing cues in our decision environment is a more effective way of directing behavior than giving information about costs and benefits or installing financial incentives. A nudge is "any aspect of the choice architecture that alters people's behavior in a predictable way without forbidding any options or significantly changing their economic incentives" (Thaler \& Sunstein, 2008, p. 6). Since Thaler and Sunstein (2008) published the book Nudge, improving decisions about health, wealth and happiness, it is seen as the new and effective way to change unhealthy or otherwise undesirable behavior through policy measures (Bovens, 2008; Hausman \& Welch, 2010). From providing information about risks and benefits and installing fines and financial incentives, policy makers and managers have become choice architects, using visual, textual and olfactory primes as tools for achieving behavioral changes.

Specific about nudging, and about Primary Care Plus, is that it supposedly intervenes in our unconscious processes of action. This idea is based on the dual process theory (Oliver, 2013), developed by Kahneman (2011). The dual process theory

18 Meeting, June 29, 2015

19 Interview, March 18, 2015 
is situationist: Most of our actions are immediate responses to cues in our environment. When responding to these cues, we follow our biases, making this way of acting automatic, fast and effective. Without system one, the theory goes, it would be impossible to take the many small and habitual decisions that are part of our daily life. However, when we need to do a complicated calculation for example, we stop and think before we act. This is when we use system two, the deliberative system. It is slow and considerate, it makes us hesitate, consider the options and weigh them before we make our choice. When we need people to eat healthy, it is more effective to intervene in the automatic system by seducing them to habitually pick an apple at eye level than to address their deliberative system by providing information about risks and benefits.

According to Thaler and Sunstein, we can predict how we respond to cues on the basis of scientific findings about our cognitive biases. For example, because we know about inertia bias, we can predict that many of the people filling in a retirement plan form will not deviate from the default plan, even though this plan will not give them a sufficient income later on. Policy makers should use such knowledge by intentionally designing choice environments in such a way that people will make "better" decisions when they act automatically. Similarly, in the hospital, patient and specialist will follow the default behavior of the hospital setting, which, according to the managers of Primary Care Plus, entails the expectation to go to the bottom of any medical problem. Relocating patient and specialist will thus take them out of this pattern of unfavorable automatic behavior. Another example is that we keep eating from a bowl of nuts placed in front of us. Translated to the hospital this means that specialists and patients make treatment decisions that are medically redundant when they are easily accessible in the hospital. This is related to what behavioral psychologists call "the temptation bias".

In a nutshell, a nudge is designed to go against automatic unwanted behavior. In the case of Primary Care Plus, the unhealthy food in the equation are the diagnostic tests; by taking them out of view, so to say, patients and specialists are steered away from the automatic act of using them. In the primary care environment of the Primary Care Plus center, it is "immediately clear for the specialist" to not "pull out all the stops", the director explained in an introductory meeting with possible new partners. This is more effective than just asking specialists to be more critical when ordering tests: "If we would just ask specialists to order fewer diagnostic tests, they would agree," one of the managers explained, "but ultimately, they would keep ordering tests on a large scale." Such promises are thus based on the idea that you can change behavior intentionally by reversing the temptation bias. Whether it is a bowl of nuts or an expensive diagnostic procedure, when somebody makes it less 
easily available, people will be less inclined to take, order or demand it. As such, the project is directed at finding new ways of making health care more sustainable and thus keeping health care accessible in the future.

Thaler and Sunstein (2008) primarily use examples of health and lifestyle choices when they discuss the policy instrument of nudging. Since the publication of Nudge!, however, the use of nudges has spread into other practices as well. A large and growing body of literature has focused on the use of behavioral economics tools such as nudging in health care. Many of these articles are explorative. They present ways to translate findings from behavioral economics into solutions for specific health care problems, such as adherence, prevention of diabetes, underutilization of reproductive health care services, vaccination, appropriate prescription of antibiotics and hand hygiene (Courtney et al., 2014; Drews et al., 2012; Roe et al., 2020; Soler et al., 2018; Williams et al., 2018). Others have focused on the effectiveness of nudges that have already been implemented in hospital or clinics (Birnbach et al., 2013; Drees et al., 2015; Johnson et al., 2016)

Besides the enthusiastic reception of the use of nudges in health care practices, some authors have also formulated possible points of critique on the use of nudging in such a context. The most often raised ethical question is whether nudging goes against the autonomy of people (Aggarwal et al., 2014; Blumenthal-Barby \& Burroughs, 2012; Navin, 2017). Since nudging interferes in the automatic behavior of people, it bypasses their rational capacities and could thus amount to a form of manipulation (Reach, 2016). This would be problematic, because for both doctors and patients, autonomy is considered a key value. ${ }^{20}$

While especially pertinent to the health care context, the question whether nudging is detrimental to autonomy applies to any other context as well. Thaler and Sunstein anticipated it in the book itself. They give two reasons why, even though nudging has an influence on the choices that people make, it is nevertheless not a limitation of their autonomy. First of all, a nudge is a gentle push and not more than that. It is like using a GPS. You can follow the directions, but you can always choose to go left when you are supposed to go right (Sunstein, 2014). That is why it is called a "liberty-preserving approach" (Sunstein, 2014, p. 583). Secondly, Thaler and Sunstein explain that even though a nudge interferes in the behavior of people without them necessarily being aware of it, it is nevertheless a legitimate policy or management instrument. It merely assists people in making the rational decision. According to Thaler and Sunstein, this means that it makes them choose the option "that will make them better off, as judged by themselves" (2008, p. 5). This is

20 This is an important question, because, as mentioned in the previous chapter, there has been a move from the paternalistic model of health care provision, in which the doctor decides the treatment, to a more patient-centered, or democratic model, in which patients are involved as much as possible in the decision-making procedure. 
an important claim, because it is the condition for a nudge to function as a legitimate health care management tool and as such a crucial part of seeing Primary Care Plus' third promise as legitimate to begin with. Nudging is different from ordinary manipulation, such as the commercial strategy to put chocolate bars on eye level. Any environment has a choice architecture. What is wrong with changing it in such a way that the best choices will most likely be made?

Making sense of Primary Care Plus' promise of cost-efficiency through relocation as a form of nudging helps seeing what notions of knowledge, agency and morality are implied in the rationale behind it. The dual process theory was developed out of a dissatisfaction with the strongly rationalized notions of agency, and relatedly, its isolation from the material and social context in which they act, in the social theories of sociology, economics and the humanities (Kahneman, 2011). The leading imaginary example in Thaler and Sunstein's book of how nudging works is a story about Carolyn, a manager of school cafeterias, who one day finds out she can significantly improve the healthy food intake of kids by changing how food items are placed in the canteen. In the next paragraphs I will use Carolyn's story to carve out the implied notions of knowledge, agency and morality.

First of all, the premise of the story of Carolyn fits the duality of knowledge and action. This is implied in the setting of the story, which presupposes a realm of knowledge in which Carolyn's idea resides on how to direct children's food choices that are conducted in the realm of reality. In terms of nudging theory, there is a duality of nudger and nudgee. Carolyn, the nudger, is a designer of nudges. The kids making food choices are the nudgees, since they are directed through slight changes in their environment.

Secondly, nudging theory is built around a specific understanding of agency, as is given in the name of Kahneman's theory, dual process theory. Conceptualizing the two ways of acting as two different processes implies there is a fundamental separation between the two. Actions are thus either thoughtless and immediate responses to the environment or well-thought out consequences of a mental process of weighing options; an action is never something in between. In the first instance, presented as a radical departure from the strongly rationalized notions of agency in the social sciences, economics and some of the humanities, a second look confirms that this difference is only partial. Agents do not always act rationally, as many experiments in behavioral psychology have shown (Haselton et al., 2015; Kahneman, 2011; Tversky \& Kahneman, 1974), i.e. their rationality is bounded, but when taking time and space, it is possible for them to turn their deliberative process on and think like a fully rational and informed human being.

This second duality is crucial for the theory's consistency and for rebuking the 
critique that nudging is a form of illegitimate manipulation, because it allows for distinguishing what people do on the one hand and what they actually want on the other. This split is not very much unlike the split between the empirical self and the categorical self as found in the work of Kant (1781/2007), one of the founding fathers of modern rationalism. While the first is the daily self, who is being seduced by circumstances and who follows emotions and lusts, the latter is the pure self, who follows reasons that are not only valid in the specific context in which the decision is made but in general. The situatedness of nudging's concept of agency is thus only partial as well. Any normal functioning human being acts automatically and in response to the environment but is also able to switch on a process of reasoning that entails separating the mind from the arbitrary environment. The Kantian subject, the agent in nudging theory, and the homo economicus that the nudging theory offered an alternative to, each fit the dualism of body and mind explained in chapter one, even though the first two only partially. In this dualism, the body is the executor of commands of the mind, that not always does, but that nevertheless has the capacity to function in isolation of the body and the empirical environment.

In theory, the two processes are often explained as processes taking place in one and the same agent. Yet, in the story of Carolyn and in many of the other examples scattered through Thaler and Sunstein's (2008) book and the articles about nudging that appeared in the past 15 years, the duality of agency structures the relation between the different players in the nudging stories. This is visible in the fact that this duality as a rule is aligned with the duality of design and action. While the nudgee's rationality is fundamentally bounded, the nudger is able to have a clear and rational mind when in the process of arranging a choice architecture. While Carolyn has the time, information and space to consider all pros and cons of eating an apple versus a hamburger with fries, in the context of buying their meals, the kids merely rely on their automatic responses to environmental cues for making choices.

With the Kantian notion of subjectivity comes a Kantian notion of morality. As explained above, in the story of Carolyn, the right thing to do for her is to help the kids take the right decisions, which means assisting them in following their true preferences. Nowhere in the book, nor in articles, Sunstein and Thaler propose to hold a vote or to research empirically what the true preferences of the nudgees actually are. ${ }^{21}$ In nudging theory, the right choice in a decision situation is the rational choice, which can be unambiguously distinguished from the non-rational one. As

\footnotetext{
21 This fits the Kantian subject, because her rationality is not empirical; it transcends the here and now. However, in literature about nudging, the discussion on how to determine such actual preferences is not completely lacking. Schiavone et al. (2014) argue on the basis of (Sunstein \& Ullman-Margalit, 1999) that proponents of the theory of Libertarian Paternalism would advocate the actual preference for people to be the option that "maximize welfare, all things considered" (2014, p. 104). Schiavone et al. themselves argue for a "preliminary deliberative debate" (2014, p. 103).
} 
such, the rational choice is the outcome of a logical procedure, irrespective of who executes it in which context, conducted by somebody's deliberative system. At the same time, however, Thaler and Sunstein assume that the outcome of such a procedure - what the nudgee would have done if she had used her deliberative system - are the true preferences of a nudgee. In the theory of nudging, morality, as the set of norms and rules that determine the distinction between a right and a wrong choice, is thus both located outside any particular situation as a general abstraction and inside the authentic, rational self.

The notion of morality in the second and in the third promise of Primary Care Plus denote morality as representation. In the promise of patient-centeredness, morality is a yardstick residing in the realm of morality, representing what good or better care in terms of patient-centeredness would entail in the realm of action. In the case of nudging, the moral norms and preferences that are both the outcomes of a rational procedure and reside in the deliberative mind are a representation of what would be the right thing to do in the realm of action. As such, both in Kantian ethics as in nudging theory, the rational self functions as a regulatory idea.

The third promise as well as the second and first are thus phrased in the representational idiom. Adapting this idiom to the notion of morality, true and actual preferences, or in the context of Primary Care Plus, the aim of avoiding unnecessary referrals while safeguarding the quality of care, and the criteria of patient centeredness, are a body of moral norms. Within Pickering's representational idiom, science is the activity of making the body of science more and more representational to the world out there. In context of nudging, designing a nudge and implementing it is an activity of making the behavior of nudgees increasingly coherent with their actual preferences. In the case of patient-centeredness, it is the activity of making the actions of specialists in the consultation room more and more in line with a predefined set of moral norms.

\section{CONCLUSION}

In this chapter, I set out to explore the rationale behind the promises of Primary Care Plus that two aspects of the intervention, interprofessional collaboration and relocation, would lead to professional learning, increased patient centeredness and cost-efficiency. I argued that the first promise of learning through collaboration resonates with the current trend in health care innovation to understand integration of care as a solution to many of its problems. The second promise, improved patient experience, lines up with the increasing importance of putting the patient and her needs center stage when providing health care. Thirdly, the promise of cost-efficiency through relocation follows the logic of nudging. The health care trends that 
I linked with the promises of Primary Care Plus helped to identify the notions of agency, knowledge and morality implied, because internationally researched trends such as Integrated Care, patient-centered care and nudging are more extensively theorized than a local health care innovation such as Primary Care Plus.

Reflecting on the three promises and combined trends, I found that each of the promises relies on the duality of knowledge and action. The promise of Integrated Care assumes a notion of medical knowledge as representative of the human body and the activity of integrating care as an activity with, among other effects, going against the fragmentation of medical knowledge in health care practices. The notion of knowledge in the promise of improved patient-experiences became apparent in the method of measuring it. Using an internationally developed index of patient-centered items, the university research team presented a representation of the patient centeredness of the Primary Care Plus in practice structures and expressed in five aspects. The third promise, cost-effectiveness through relocation, following the rationale of nudging, presumes a duality of knowledge and action, because it fundamentally distinguishes the level of the nudger, the designer of the nudge, from the level of the nudgee, the person being assisted in taking the right decision.

The second notion I carved out of the promises and trends is the notion of morality. In both the second and the third promise, patient-centeredness and nudging, I found a notion of morality as representation. Such a notion of morality implies a separation between the realm of morality, where moral norms such as criteria for patient-centeredness or the actual preferences of the nudgee reside, and the realm of action, in which actions unfold that do or do not comply to the norms of the realm of morality.

Last but not least, the theory of nudging gave insight into the notion of agency implied. The nudging agent is, against how it is presented in literature, not a radical alternative to the rationalized notions of agency such as the homo economicus. Both are acting bodies, (sometimes) directed by a mind capable of rationally weighing pros and cons in isolation. In the third promise, and in nudging theory, nudgees, or doctors and patients, are conceptualized as empirical bodies responding automatically to directly observable cues in the environment. However, the legitimacy of the relocation as a nudge is thus only legitimate when presuming them to be Kantian subjects, possessors of true preferences, that can guide the direction of a legitimate nudge.

The promises of Primary Care Plus and the outcome measures used by the university research team to research whether the intervention entailed an improvement or not, are both sources of explicit knowledge about the intervention reality. In the next chapter, I will shift focus from the explicit to the implicit. Instead of exploring 
what the intervention is promised to be, or instead of measuring its outcomes, I will focus on what was done there and then, i.e. on what professionals do when managing Primary Care Plus. More specifically, in the next chapter, instead of taking the interprofessional collaboration as a way of integrating care and medical knowledge, I will focus on what the knowledge actions of "integrated-care-in-action" entail. This will help create a broader and richer understanding of what Primary Care Plus and Integrated Care entails in practice. 


\section{3}

\section{INTEGRATED-CARE-IN-ACTION THE KNOWLEDGE WORK OF RESOLVING AND ACCOMMODATING DIFFERENCES}

\section{Interpreting incontinence 22}

"Incontinence", the daily manager of the Primary Care Plus centers reads out loud from a slide projected on a screen in the meeting room where the bimonthly users' council meeting takes place. "We have decided that patients with incontinence problems need to be referred to the outpatient clinic of the hospital and not to the center." The rectangular tables in the meeting room in the Primary Care Plus center East are placed in a U-shape. At the tables making up the legs of the U-shape, a hospital manager, four general practitioners, three medical specialists and three university researchers are seated. The daily manager of the Primary Care Plus center is sitting at the bottom of the U-shape facing the projection screen. During the users' council meeting general, practitioners involved in the organization of the project and medical specialists who regularly work at the center discuss practical problems and new plans with the daily manager. The researchers, of whom I am one, are from a multidisciplinary research group from the university. Two of the researchers are there to present preliminary results; I am present at the meeting to take ethnographic observations.

Right now, the manager presents a list composed by the gynecology working group to help general practitioners refer the right patients to the gynecology consultation hours of the Primary Care Plus center. The projected slide presents three columns under the heading "gynecology": one with medical problems that are suitable for Primary Care Plus, one with the needed diagnostic procedures and one with medical problems unsuitable for the center. After the daily manager had read out loud that patients with incontinence need to be sent not to the center but to the outpatient clinic, one of the general practitioners informs: "Should we send those patients to the gynecology or to the urology clinic [at the hospital]?" "Gynecology," the manager says decisively. "Well," the urologist present at the meetings interrupts, "I would say urology."

22 Meeting room, March 23, 2016, 5:30-7.00 PM 
The urologist's intervention causes the general practitioners and specialists to laugh. The manager turns her face to the urologist and says with a friendly and much less decisive tone of voice: "Yes, that is possible too." The urologist further elaborates: "Incontinence problems belong at the urology department. But I know a gynecologist would probably say it is part of gynecology." Another general practitioner adds: "They say that men should be referred to the urologist and women to the gynecologist." The urologist disagrees: "Many women with incontinence have other problems than just stress incontinence. They really belong at the urology department."

The room falls silent. A third general practitioner: "Well, this is difficult for us. That we uhm..." The urologist, now attempting to appease the disagreement: "Of course it is fine to hold different opinions." Manager: "Of course!” The third general practitioner starts formulating a response: "But we, well, we have to... uhm..." She cannot finish her sentence, because a fourth general practitioner jumps in: "Before you know, the situation will become like when we need to decide between referring a patient to the surgery or the orthopedics department! You need to first check which day of the week it is to know which clinic to choose. That is so ridiculous." Then, addressing another present general practitioner: "Don't you think, Astrid?" This is when an uncoordinated discussion between the present doctors erupts. After a couple of minutes, when the excitement dies down, the manager jumps in with a new topic of concern for the gynecology consultations at the center.

On first sight, these brief observations depict an unremarkable instance of diverse medical professionals involved in managing the delivery of Primary Care Plus care. Yet, if we place these interactions in the framework of Primary Care Plus as Integrated Care as explained and analyzed in the previous chapter, some telling contradictions come into view. Primary Care Plus is a new model of health care delivery, in which doctors from different medical specialties and regional general practitioners work together to improve specialist care for less complex problems. The vertical collaboration between primary (general practitioners) and secondary (medical specialists) care providers is one of the unique selling points of the intervention. Collaboration helps participating professionals to extend and broaden their medical knowledge. In the previous chapter, I called this "the first promise of Primary Care Plus", which I identified as fitting the current trend of health care integration.

Integrated Care and as such the first promise of Primary Care Plus, I argued, are phrased in what Pickering (1995) calls the representational idiom. Adapted to the health care context, this means that medical knowledge is seen as a representation 
of the human body, its bodily processes and interactions with the environment. The rise of specializations, and the fragmentation of medical knowledge, is viewed as a problem that can be countered by integrating the different medical knowledge practices and working towards a coherent and representative body of knowledge. ${ }^{23}$ In such an approach to interprofessional collaboration in health care, knowledge is seen as a product, a commodity, that can be possessed and transferred.

The above observations are a snapshot of interprofessional collaboration. In the framework of Integrated Care, it is expected that through collaboration, professionals will gain knowledge. Yet, in the above excerpt, it shows how conflicting knowledge slows down the organizational process. In this instance of collaboration, knowledge is not a commodity that can go from one to the other but a problem, a hurdle in the process of organizing. Within the representational idiom, it would be expected that the process of care integration entails resolving such a conflict to restore coherence. But this is not what happens in the above excerpt. On the contrary, the professionals in these observations are not countering fragmentation but accommodating it. They are not taking away knowledge conflicts but making space for them. Is this then an example of failed health care integration? Or a moment when the process slowed down, amidst other moments in which the efforts where more successful?

In this chapter, I take up the performative idiom (Pickering, 1995) to allow for a different understanding of the processes occurring above. The discussion unfolding in the observations, and others similar to it, do not need to be dismissed as damaging instances where health care and knowledge integration stalled, but they then become an important source of insight into what professionals do when collaborating despite knowledge differences. In the performative idiom, it is acknowledged that not only humans but also materials, such as the indication lists, or collectives, such as the users' council meeting and its interactions, have agencies as well and that these are emerging instead of designed and predictable. In the context of this study, knowledge is not (merely) the intended learning effect that would follow naturally from drawing up a plan (design), taking care of facilities such as renting the building in which the consultations take place, making a schedule for specialist consultation hours and implementing these (execution of the design). Rather, it is comprised of emerging knowledge actions, i.e. the ongoing efforts in the knowledge practice of Primary Care Plus to enable integrated health care delivery. Designing a model and

23 The term and research area of Integrated Care also implies a generative metaphor (Schön \& Rein, 1995, p. 28). Schon and Rein call this the "metaphor of wholeness and fragmentation", often used in defining policy problems and solutions. By using a generative metaphor, policy makers transform a vague policy problem into a concrete one with a clear set of norms and social reality. In the context of the study, when using the term Integrated Care, health care professionals and researchers give off a clear message about how the problem should be defined, fragmentation in health care, and how it should be solved, by integrating the fragmented parts, and thus a set of norms about what good care entails. 
setting up the location is just the beginning of shaping an effective work process in which medical professionals from a variety of specialties and thus knowledge practices work together. The 'indication lists', such as the gynecology list presented during the meeting, and the observed discussion required to put such a list to work, are part and parcel of these ongoing efforts. At least as important as designing a model of Integrated Care is the continuous effort to connect, align or in this case explicate and accommodate conflicting understandings held by the involved medical professionals.

I will view the knowledge dimensions of Integrated Care to be the ongoing and emerging process of connecting, aligning and collaborating unfolding on the work floor. As such, I build on an argument made in (Glimmerveen et al., 2019): In current research, literature and policy documents on Integrated Care, authors do not always pay sufficient attention to the "processual character of integration" (Glimmerveen et al., 2019, p. 8). They argue that in Kodner and Spreeuwenberg's (2002) definition, quoted in the previous chapter, but also in reports about Integrated Care and about similar innovations in the organization of health care (Integrated Care pathways) in which telling terms such as "blueprint" (NHS Engeland, 2016) or "32 step methodology" (Vanhaecht \& Sermeus, 2002) ${ }^{24}$ are used, Integrated Care is understood as the outcome of a linear process, of unproblematically applying a method or implementing a model after it has been designed. According to Glimmerveen et al. (2019), however, such an approach takes the dynamic context of care practices out of view and relatedly, the difficult task of "juggling the disparate considerations" of the various professionals and patients involved. The above observations of the user's council meeting, that is, the efforts of explicating the different meanings of 'incontinence', suggests as well that the informal, dynamic and ongoing effort to connect and align the various professionals collaborating in a diversity of settings, such as the users' council meeting, is a crucial part of the endeavor. Further building on the brief remarks of Glimmerveen et al. (2019), I will identify and explore what I call "integrated-care-in-action", the dynamic and ongoing efforts to deal with knowledge differences in Integrated Care practices. The main question of the chapter will be, how does integrated-care-in-action, as the ongoing effort to deal with knowledge differences, take shape in the practice of managing the delivery of Primary Care Plus? By answering this question, I will shed light on how professionals achieve collaboration despite these differences.

In order to answer the research question, in the next sections, I will first further analyze the opening observations above. What kind of efforts can be discerned here? What can we learn from already existing theoretical literature about similar

24 Brought to my attention by Zuiderent-Jerak (2015). 
processes? After exploring the field, I will turn to two other discussions in the users' council meeting. In the first findings section, I analyze a discussion that emerged when the indication list as a solution to wrongly referred patients was first suggested. In the second one, I dissect a discussion about a proposal of one of the orthopedists of the hospital to relocate the spine center consultation to the Primary Care Plus center. Exploring and analyzing these discussions, I further build an understanding of what collaborating despite knowledge differences entails, and what kinds of norms it reproduces.

\section{KNOWLEDGE WORK}

Bringing forward the processual aspects of Integrated Care fits a shift of focus that can be found in a diversity of social sciences, from the formal aspects of organizations and practices to the informal ones, i.e. the taken-for-granted and ongoing work that people conduct daily. For example, in Science and Technology studies, Latour and Woolgar (1979) famously studied the laboratory work of the SALK institute, not as a formalized procedure or method of testing theories but as a process of constructing scientific facts consisting of daily activities such as circulating and transforming texts. Berg and Timmermans (2003) use a similar processual view for understanding evidence-based medicine in health care. They explicitly state that instead of discussing whether evidence-based medicine is advantageous or disadvantageous on a "rhetorical level", they focus on "what standards do in medical practice" (2003, pp. 21-22). Medical sociologist Allen (2014), as well, emphasizes not the official caring activities in the job description of nurses but the taken-for-granted but rarely described or acknowledged organization work that takes up approximately $70 \%$ of their daily activities.

Focusing on Integrated Care as a knowledge practice filled with ongoing and emerging human, material and social agencies, I am interested not in the broader plans and visions of Primary Care Plus but in the mundane activities undertaken to deal with the omnipresent and emerging agencies. In this chapter, I perform exnovation; I focus on what was done there and then (Iedema et al., 2019). This resonates with the notion of 'articulation work', coined by Strauss (1985, 1988). In his research, Strauss brought a specific category of organizational work into view: the mundane, informal and often-invisible activities involved in making organizational processes run fluently through joining the distinct parts. ${ }^{25}$ By taking the observed discussion as articulation work, I emphasize efforts such as composing indication lists together (collaborating), meeting on a late afternoon (connecting) to

25 The verb "to articulate" is commonly used to mean "to pronounce something clearly and distinctly" or "to express something fluently". In the way Strauss uses the term, it connects to its other dictionary meaning: to "consist of segments united by joints" (Merriam-Webster, 2021). 
discuss the lists, and accommodating the distinct interpretations (aligning) that are revealed through discussing it, as essential parts of integrated-care-in-action. The observations of the users' council meeting reveal that when the work is divided among workers and there is clarity about the distribution of responsibilities, the different parts still need to be "integrated" and "meshed". Postma et al. (2015, p. 65), referring to Gerson and Star (1986), and Hampson and Junor (2005), state: "Integration and meshing can be attained via formal planning and scheduling, but also requires implicit and intangible efforts, such as the bringing together of social worlds".

Taking the above observations as articulation work, an important contrast with Strauss' concept comes to view. While Strauss mainly emphasized the temporal dimension of articulation work, i.e. the efforts concerned with achieving an effective work trajectory, Bardram and Bossen (2005, p. 131) have highlighted the spatial dimension, which they call "mobility work": "the moving about of people and things as part of accomplishing tasks". Yet, the brief discussion between medical professionals about the meaning of incontinence, as described above as an instance of articulation work, is neither part of a spatial nor a temporal dimension. The work needed to connect, align or accommodate the disparate knowledge practices is different from the work of connecting and aligning different trajectories and locations. In brief, there is a knowledge dimension to articulation work as well, i.e. it also involves "the moving about of" knowledge and meaning required to enable organizational processes to run.

Aspects from Star and Griesemer's (1989) historical study of the development of a natural history museum can substantiate the knowledge dimension of articulation work. The article became famous, because the concept of "boundary object", now still widely used in fields of research as diverse as organization and management studies (Bechky, 2003; Bresnen \& Harty, 2010; Bresnen, 2009, 2010; Carlile, 2002; Engeström \& Blackler, 2005) and sociology of medicine and medical anthropology (Keshet et al., 2013; Sullivan \& Williams, 2012), was first introduced. In the article, boundary objects, such as "specimens, fieldnotes, museums and maps of particular territories” (Star \& Griesemer, 1989, p. 408) are defined and presented as objects that "inhabit (...) intersecting social worlds (...) and satisfy the informational requirements" of each of these worlds. Typically they are "plastic enough to adapt to local needs" but also "robust enough to maintain" their identity when crossing different worlds” (Star \& Griesemer, 1989, p. 393).

For the notion of knowledge work, I do not use the concept of boundary object, but I build on the main point of the article instead. Star and Griesemer (1989, p. 388) explore how it is possible for the diverse professionals, "scientists, amateur 
naturalists, patrons, hired hands and administrators", involved in the project of setting up a natural science museum to work together successfully. Their broad answer to this question is that "substantial labour" (1989, p. 388) is needed: The different professionals need to reconcile "the meanings" attributed to the "new objects and methods" crucial for building the museum, i.e. they need to "translate, negotiate, debate, triangulate and simplify" (1989, p. 389). Developing and using boundary objects is an example of such "substantial labour". They make it possible for participants from disparate knowledge practices to overcome their differences without having to change their practices.

While the work of reconciling meanings is central and visible in the history of the science museum ${ }^{26}$, I am interested in the knowledge dimension of the Primary Care Plus project's articulation work, which, on the contrary, is mundane and invisible. This dimension, which I denote with the term "knowledge work", is thus a combination of articulation work and the substantial labor of reconciling meaning described by Star and Griesemer. Knowledge work denotes the mundane and invisible activities concerned with differing knowledge practices, required for making the collaborative work processes to run well.

As an instance of work necessary to make collaboration across professional boundaries possible, the concept of knowledge work resonates with the term "boundary work”, originally coined by Gieryn (1983, p. 781) as the work conducted by scientists to "demarcate science from other intellectual activities". While Gieryn's work is aimed at placing and maintaining boundaries, the work described in this chapter, in contrast, is aimed at lubricating the process of working across them. Instead of emphasizing differences on both sides of the boundaries, the work is aimed at navigating, accommodating or resolving differences. In this sense it fits the category of "collaborative boundary work" defined by Langley et al. (2019, p. 714) as the practices that "emerge as people work in interoccupational teams, produce services, and construct interorganizational collaboration". Literature about collaborative boundary work categorized by Langley et al. (2019, p. 714), (Apesoa-Varano, 2013; Barrett et al., 2012; Bechky, 2006; Quick \& Feldman, 2014), describes "how boundaries are negotiated, aligned, accommodated and downplayed to get the work done".

\section{KNOWLEDGE AS PRACTICE}

In the previous chapter and in the above section, I did not only point out how the promise of learning through collaboration was phrased in the representational idiom but, relatedly, that it comes with the assumption of knowledge as commodity

2680 Years later the methodology developed by the Grinnell (biologist and founding director of the museum) to archive objects is still being used in natural history museums (Star \& Griesemer, 1989). 
or as a mentalist view. The knowledge actors in these claims, but also in Integrated Care literature, are seen as possessors and users of knowledge, or they mention that specific knowledge needs to be obtained for functioning well in Integrated Care. Knowledge itself is a commodity, a product, that can unproblematically be transferred from one setting to the other and from one knowledge actor to the other, without it being transformed. Taking the practice of managing Primary Care Plus as a "mangle of practice" (Pickering, 1995, p. 23) means taking on an entirely different conception of knowledge that Gherardi (2000) calls knowledge as practice. Rather than an object-like entity that is possessed and that can be unproblematically transferred, or as something that resides in the mind and can be obtained through mentalist processes of learning, knowledge is taken to be a situated activity (Gherardi, 2014). Such a conception of knowledge has four important implications, which allow for a different understanding of the above observations.

First of all, in the above observations the term 'incontinence' as a medical problem acquires a specific meaning not (only) as a representation of a medical problem in the human body but also as part of the indication list as referral instruction under discussion among medical professionals from different knowledge practices. Knowledge, in this case about incontinence as a medical problem, is thus not (merely) a representation that we consult when we need to act, but it is an action itself, shaping the practice of dealing with and discussing incontinence. Gherardi (2000) refers to what Heidegger (1962) and (other) phenomenologists have called Dasein, the being-in-the-world in which subject and object cannot be distinguished. If we take the stock Heideggerian example of the hammer ${ }^{27}$ : When we observe it without using it, we will not acquire much knowledge about it. Only when we pick it up and use it, we will fully understand what a hammer is. Similarly, the knowledge work of the participants of the users' council meeting cannot be extracted from these knowledge practices. For example, the way the different meanings of the term incontinence are being addressed and accommodated as two different understandings of how to direct flows of patients with one specific symptom is unthinkable in a setting such as a consultation room, in which a doctor confers with an individual patient what to do about losing urine regularly. The knowledge about the hammer is as much part of the practice, i.e. the activities involved in hammering, as the hammer itself.

Secondly, isolating the problem of incontinence from the practice in which it is

27 It could be objected that this example is not exemplary, because it is about a specific kind of knowledge; tacit knowledge. Adopting the notion of knowledge as practice, however, means accepting that any knowledge is bound up with activities and is thus always more or less tacit. Hammering in a nail, Heidegger's often paraphrased example, might be at the one end of the scale and managing Primary Care Plus at the other, but this is not a principled distinction; it is a matter of degree. 
enacted will inevitably modify its meaning. By reflecting on the action, we could write down an account of such knowledge, but this would not be complete, nor would it be precisely the same as the knowledge gained through participating in the activity of discussing what should be the appropriate trajectory for patients with incontinence problems. Knowledge cannot be isolated from its context, its activities, and/or its material setting without being transformed. The knowledge that the carpenter has gained from using the hammer cannot be separated completely from the action itself. By thus taking knowledge to be a situated activity, I take it as inextricably bound up with the practice in which it is conducted, as inscribed in our material practices and bodies. Going back to the indication list again, the observations show how, through the transferal from working group, where the indication list was composed, to the slideshow presentation and object of discussion in the users' council meeting, it once and again acquired a different meaning dependent on the setting and activity in which it was enacted. The process of acquiring or transferring knowledge is thus not neutral; it will always leave a mark. Because knowledge is an activity, inextricably bound up with the context in which it is performed, it will be different during and after a process of transferal or acquisition.

Thridly, in the collaborative effort of making the list, but also in transferring it to a slideshow presentation and discussing it with new professionals, knowledge is produced about, for example, the list itself or about the practical problems for medical professionals dealing with these problems. Thus, each instance of knowledge activity produces new knowledge.

A fourth implication of understanding knowledge as a situated activity is that it is performative, i.e. that it shapes the world in which it is conducted (Rouse, 2001). This implication is related to how practice scholars understand the term practice. In its most basic sense, a practice is a set of activities (Schatzki, 2001a, p. 11). However, in order to conceive a set of activities as a practice, some coherence between the different activities in a set is implied. Practice scholars explain such coherence in two different ways: first, as constituted by the taken-for-granted routines in which automatic or habitual activities are embedded, such as the training of bodies in which the routines are inscribed (Swidler, 2001); second, by understanding practices normatively (Rouse, 2001). Practitioners do not act uniformly, neitherare their beliefs strictly the same. A practice is held together by the fact that the actions of its participants are answerable to norms of correctness. Being answerable, however, does not mean deviant behavior is always sanctioned or corrected. The responses to correcting and not correcting behavior are normative practices by itself, that is, the actions that make up such a practice are answerable to norms as well. With this view, norms are not reified but are continuously reproduced and shaped by practitioners' acts 
and responses to acts of others that follow or defy the norms.

Within the normative conception of practice, knowledge activities are also answerable to norms and, thus, they reproduce and produce norms about, for example, what counts as correct and incorrect knowledge or what counts as a sufficiently reconciled knowledge difference. For example, with the presentation of the list, but also with the responses to the presentation, such as the discussion that erupted about the meaning of incontinence, norms about what counts as a correct referral or of a correct understanding of incontinence (and other medical problems) are produced and reproduced. In this light, the decision of the manager not to fully align meanings of incontinence but to merely accommodate the difference is an example of producing a norm about to what extent such reconciliation should be achieved. As such, the knowledge work conducted in the users' council meeting does not only shape and transform knowledge norms, but it also shapes the norms that constitute relations among the involved professionals and the ones by which practitioners distinguish correct from incorrect practices. In sum, these knowledge activities are performative.

Conceiving knowledge as practice is a further substantiation of the notion of knowledge work introduced in the previous section as the ongoing mundane and often invisible efforts of reconciling disparate knowledge practices sufficiently to enable organizational processes to run. Even though both the work of Strauss and of Star and Griesemer were written when the terms practice and practice approach were not yet widely used, they should be seen as part of the broader turn in the social science from focusing on 'structures' or 'actions' to 'practices' (Reckwitz, 2002). As one of the main scholars explicating the practice approach, Gherardi defines Strauss' notion of articulation work as "the set of activities necessary to accomplish a working practice" (Gherardi, 2014). In their article about the Californian science museum, Star and Griesemer (1989) move away from what they call "common myths" of science, towards a close examination of scientific work, the set of activities conducted by "scientists and other actors" who "translate, negotiate, debate, triangulate and simplify in order to work together". In the article, they do not explicitly mention the term "practice", but they do explain their analysis to be "ecological" for not prioritizing one viewpoint over any other. This is very similar to the practice approach, in which, as explained above, knowledge activities such as hammering are as much part of the world as the hammer and the arm of the carpenter. In approaching integrated-care-in-action as entailing acts of knowledge work, I take the knowledge practices that clash within the intervention of Primary Care Plus and the efforts of dealing with conflicts between practices as dynamic and ongoing knowledge activities. The knowledge of interest in this chapter is not a 
commodity being transferred, it consists of continuously (re)produced knowledge acts involved in collaborating across professional boundaries. By viewing knowledge activities as such, production and reproduction of norms, i.e. the performativity of the activities and thus its implications, are foregrounded. This helps bring into view how professionals navigate existing and emerging differences and the effects of how they deal with it.

In viewing the users' council meetings' as a knowledge practice, knowledge can be viewed as part of the same world as the chairs and tables in the meeting room, the slideshow presentation of the manager, and the professional's response to the lists projected on the screen. The produced indication lists, for example, are not external to and representative of the world in and of themselves; they are part of it and as such constantly placed in relation with other things in the world. They are not representative of the world but become representative when used in practice as "models with an intentional relation to what they model" (Rouse, 2001, p. 203), because then they are taken up in "ongoing patterns of use that are answerable to norms of correctness".

To summarize the above, knowledge work, i.e. the ongoing mundane and often invisible efforts of dealing with differing knowledge practices to enable the delivery of collaborative care, is an essential part of Integrated Care. This, I have shown, should be seen as another dimension of articulation work, aside from the temporal and spatial ones, i.e. the work that enabled processes to run fluently through joining the distinct knowledge practices. Tracking the knowledge work of Integrated Care as knowledge as practice creates a sensitivity to, first of all, how knowledges are shaped and reshaped, and secondly, how the knowledge activities are performative, that is, how they thus constitute relationships among different practitioners and norms about what counts as good care and what not. This gives a basis for answering the research question, to see how integrated-care-in-action takes shape in the practice of managing the delivery of Primary Care Plus.

In the next two sections, I will further track and analyze the knowledge work conducted during two users' council meetings of the Primary Care Plus centers. As explained above, viewing the users' council meetings as knowledge practices "filled up with agencies" means understanding knowledge as a situated activity. I will thus focus on the knowledge (inter)actions of the professionals in the users' council meeting, i.e. the verbal and non-verbal actions that shape or reshape how they understand and assess the world around them. To be more specific, I will focus on knowledge work, the knowledge dimension of articulation work, as a specific kind of knowledge activity. The two discussions explored in detail are selected, because they are clear examples of integrated-care-in-action, i.e. they entail incompatible 
knowledge actions that are dealt with in a subsequent process of knowledge work. In each case, I will first describe the contrasting knowledge actions that stand in the way of defining and solving organizational problems. Secondly, I will disentangle the knowledge work conducted to achieve sufficient reconciliation. In the course of describing these knowledge actions, I will reflect on the normativity and the performativity of the described actions.

\section{JUGGLING PROBLEM AND SOLUTION: INDICATION LISTS AS A SOLUTION IN THE MAKING}

In this subsection, I will look into a discussion related to indication lists as I did in the introduction of the chapter. For the analysis in this subsection, however, I take a step back in time. The meeting described in the introduction took place in Spring of 2016, when teams of medical specialists and general practitioners had been working together to compose the lists. The observations gave a view of the interactions that occurred after the daily manager of the centers presented a list composed by one of the teams.

In this section, instead, I will turn to a users' council meeting of more than a year earlier. ${ }^{28}$ This was the first of the meetings observed during the ethnographic fieldwork in which the indication lists, here still called "referral protocols", were mentioned as a solution to an organizational problem. It was early 2015, the users' council was still rather small. The meeting was attended by two general practitioners (GP1, GP2), three medical specialists, a rheumatologist (SP1) and two who did not participate in the discussion. Also, two professionals with a managerial role (M1, M2) attended the meeting and two researchers of the university research team, of whom I was one, who also did not participate in the discussion. The daily manager, who chaired the meeting described in the introduction, had not been hired yet. Instead, the meeting was chaired by a project manager from the hospital (M2). Also, the director of the regional primary care organization (M1), who had developed the concept of Primary Care Plus and had initiated the collaboration with the hospital, the insurance company and the patient organization of the project, attended the meeting.

\section{Inefficient consultation hours - system or knowledge problem?}

During the meeting, the rheumatologist, SP1, who frequently works at the center brings up the inefficiency of her Primary Care Plus consultation hours: "Right now, I am not able to do anything else while running my own consultation hours. These are full consultations. It is hard work to deal with the patients' problems within the dedicated time." One of the general practitioners, GP1, asks: "If I look at your schedule here, is it correct that you see eight patients every shift...?" SP1: "Yes, I see

28 Meeting room, February 5, 2015, 5:30-7.00 PM 
eight new patients when I am here. (...) that is more than the average at the hospital. It is fine, but I cannot also supervise a fibromyalgia consultation hour. (...) unless we look at what kinds of patients are coming in. Some of them are at the right place, but some...” In the silent moment that follows, SP1 casts down her eyes, showing vicarious shame. Then, she sits up and says: "Nobody thought I would have to see these kinds of patients here! Not you, not me, nobody! New cases of rheumatoid arthritis, new cases of ankylosing spondylitis, young children... Only a month ago, a 14-year-old boy with childhood arthritis, which is very rare, was sitting here with his mother and father... it was such a wretched situation!” (...) While M1 suggests a solution - Tthis shows how important it is that we start working on the referral protocols" - SP1 continues her story without paying attention to the director's suggestion: "Those [faulty] referrals... these are our bottle neck. I understand the system, but this is so frustrating..." The other doctors fall silent, seemingly moved by her anecdote. M1 is the first one to break the silence by elaborating on the solution he just proposed: "We need to examine per specialty what kinds of medical problems are suitable for the center and what kinds are not, what belongs here and what not... And we need to put an effort in implementing this broadly among primary care providers. It needs to become crystal-clear which patient should and which should not [be sent here]."

The above description of a mundane exchange of heartfelt experiences, emphatic silences and quickly suggested practical solutions brings into view some of the contrasting knowledge actions pertaining to problematic Primary Care Plus referrals of general practitioners. The problem was not a designated item on the agenda; the rheumatologist, SP1, brought it up as a response to the manager asking her to plan more rheumatology consultation hours at the center. She is reluctant to relocate her efficiently organized consultation hours at the hospital to one of the centers, because here, due to the many wrongly referred patients, it is impossible to handle her consultation hours efficiently.

The knowledge interactions in this excerpt are not neutral transferals of knowledge. The way in which SP1 brings up the problem, recounting the anecdote of a 14-year old boy with childhood arthritis, accompanied with the described body language, and bringing in "the system" as a passive but decisive player, and the silent but significant response of the general practitioners, produce a specific problem reality. With the knowledge action, SP1 has conveyed her frustration with the situation but has also set some norms about what a good referral practice entails, how it can be achieved in theory and in practice. In this problem reality, misplaced patients are at the core of the problem. These patients have such exceptional and complex 
medical problems that the specialist cannot take care of them in the short consultation hours at the center, thus causing the consultation hours and/or the patient trajectory to become inefficient. A good referral practice is one in which consultation hours would run predictably and routine-like, exempt from such astonishing cases, and patients would go through a linear care trajectory, in which no time would be lost because of consulting a specialist at the wrong location. This situation cannot be achieved by changing the general practitioners' behavior - an unrealistic aim according to SP1 - but only by changing the system, which, SP1 found out in earlier exchanges with the management, is not an option..$^{29} \mathrm{By}$ blaming a system that cannot be changed, SP1 produces a norm prescribing a rather passive and fatalistic attitude towards such referral problems.

The director's knowledge action contrasts with the anecdote recounted by the rheumatologist. He brings in the practical solution of composing referral protocols that should help general practitioners send the right patients to the Primary Care Plus center. By bringing in this specific solution, he reshapes the problem reality: Instead of dramatic but inevitable aspect of the system, it now becomes a simple knowledge deficiency on the side of the general practitioners, easily solved by providing the missing pieces of information. The new problem reality is accompanied by a different set of norms. Good referral within this problem reality can be achieved by correctly following a protocol that links medical problems to appropriate decisions. This implies that correct referral is something that, in the first place, can be distinguished from incorrect referral by merely looking at the information about the medical problem of the patient, collected by the general practitioner during the consultation at the general practice. These knowledge actions thus produce a norm that designing protocols is the appropriate way of solving these kinds of problems.

It is clear now that the organizational hurdle discussed in the observations has a plural reality. Within the understanding of knowledge as practice, the dichotomy between reality out there and knowledge representing that reality is rejected. Knowledge is not (merely) a representation of the world in which people act, but it is a situated activity, part of that world and thus co-shaping it. This is what I called the "performativity of knowledge (acts)" in the previous section. From this follows that the contrasting knowledge actions of SP1, recounting the dramatic anecdote, and GP1 and GP2 responding with an emphatic silence on the one hand and M1 inserting the quick and easy solution of providing information on the other, do not

29 In a later interview, the rheumatologist explained that when she decided to collaborate with the Primary Care Plus project, she had anticipated another kind of rheumatologist care delivery. She wanted to organize a walk-in clinic: Patients would just stop by, show their hands, and after merely examining their hands in a limited amount of time, she would filter out the patients who actually need specialist care at the outpatient clinic of the hospital and those who do not. 
just imply different representations or perspectives on a problem in the actual world out there; they create different problem realities: a system problem and a problem of knowledge deficiency. What counts as the problem during the interactions between the different professionals attending the meeting is crucial, because what can be conceived as an appropriate solution depends on the problem reality created by the knowledge activities (Gusfield, 1981).

\section{A new set of players: TIPP employees}

One of the two general practitioners, GP2, is clearly not convinced that the solution proposed by the director will solve the problem. He starts breaking down the work process preceding the moment described in the anecdote: "The referral letter arrives at TIPP [organization that processes the secondary care referrals made by general practitioners in the region]. How is it possible that TIPP places such a patient [14-year-old boy with childhood arthritis] at the Primary Care Plus center?

For M1 there is only one answer: "Because the general practitioner gave it as an option [in the referral letter]." But again, he does not get full support of the present doctors. "I cannot imagine a general practitioner saying 'this 14-year-old-boy, let's send him to the Primary Care Plus center!'," SP1 says. GP2 suggests another hitch: "It is possible [in the electronic form that general practitioners use to refer patients] to choose the option 'allow TIPP to determine where to refer the patient to'. If they [assistants working at TIPP] notice open spots in the center's rheumatology schedule, I think they just automatically fill them up [regardless of the patient's problem]."

The director, M1, attempts to take away the general practitioners' suspicions: "there are two levels of concern here. The first is the general practitioner taking the decision to refer the patient to a medical specialist. She will need a specific protocol, giving instructions about which medical problems should and which should not be sent to the center. (...) The other is that the general practitioner decides to leave the placement up to TIPP. But then, as well, there needs to be a clear instruction what can and what cannot be dealt with at the center. It is a fact that we should start debating among each other what belongs here and what not."

The two general practitioners are still not convinced that the solution fully covers the issue. GP1: "It is a good idea [to make those protocols], but it is true that TIPP also interferes in the general practitioners' decisions." GP2 confirms: "it is surely the case that TIPP has taken up a directive role." GP1: "Patients of whom we are certain they can go to the center are redirected to the hospital..." GP2 adds: "Or vice versa." 
The exchanges between general practitioner and director in the above excerpt show two things. First of all, GP2's knowledge activity, consisting of shifting the focus from the general practitioners to the employees of TIPP, clearly indicates that to him, the referral protocols that the director had suggested are not a self-evident solution for the problem at hand. However, instead of addressing the solution, his knowledge actions address the problem reality, in which he introduces a new set of players, the TIPP employees. Even though TIPP is not part of the Primary Care Plus Centers, TIPP was a topic of discussion quite often at the users' council meetings. At the call center of TIPP, the employees contact patients to make appointments for them at the hospital or with secondary care providers. After being operational for a few months, most of the assistants working at TIPP were replaced by new ones for reasons that remained unclear. This raised suspicion among general practitioners and managers. The new employees were broadly seen as incapable of making sound decisions about where the patients should be sent. Whenever there was an organizational problem, they would question the competences of the assistants working at TIPP. By bringing up the TIPP employees in this discussion, a new problem reality emerges. Now, it is not the knowledge deficiency of the general practitioners or an inevitable problem of the system but a problem of underqualified TIPP employees making dramatically incorrect referral decisions. This is a crucial knowledge action, because by changing the nature of the problem, from one of knowledge deficiency to one of competences (of TIPP personnel), what counts as an appropriate solution is modified as well. For GP2, the appropriate next step is not to make indication lists to provide the general practitioners with the knowledge that they are lacking but to take away the formal possibility for TIPP employees to independently decide where to refer a patient to. What this shows is that the fit between the problem and the offered solution is not a given; it requires knowledge work. In this case, before concrete steps can be taken to set up and execute the practical work of implementing a solution, if any, the conflicting problem realities first need to be dealt with.

Secondly, the act of breaking down the procedure preceding the anecdote's 14-year old arrival at the Primary Care Plus center is not only a negative reaction to the director's suggestion of the referral protocols as a self-evident solution but also the start of a process of knowledge work. By disentangling and explicating the steps that might be of concern for understanding what went wrong in the problematic example brought up by the rheumatologist, GP2 reveals possible joints for connecting the different problem realities. M1's knowledge activity, conducted as a response, makes use of the opportunity this creates. M1 reformulates GP2's competing problem reality in a way that it is compatible with his earlier mentioned solution, the indication lists. By appropriating parts of GP2's problem reality, he joins in 
the process of knowledge work that GP2 had started.

However, M1's act of partial appropriation does not alter the nature of the problem reality implied; in his act, it is still a matter of knowledge deficiency, not qualification. M1 and GP2 have now attempted to reconcile the different knowledge realities by conducting two different kinds of knowledge work. While GP2 disentangles and maps different procedural steps, M1 appropriates part of GP2's problem reality. Even though GP1 and GP2, at the end of the excerpt, do not have objections to "making protocols" anymore, they still do not see it as a solution to the problem. With this specific knowledge work, M1 on the one hand and GP1 and GP2 on the other have not achieved sufficient reconciliation of problem realities.

\section{Protocols for doctors and TIPP}

After GP1, GP2 and M1 bicker about who initiated the faulty decisions, going over and dismissing a handful of options. M1 again repeats his solution: "We need clear instructions that an active arthritis does not belong at Primary Care Plus center, that emergency care does not belong at the center." GP1, who is still suspicious, now proceeds with breaking down the trajectory in order to find possible hitches: "What happens if a patient calls to TIPP and says, 'I want to plan my appointment'?" M1: "It depends on what you have written in the referral letter. That is where it starts. Most of the general practitioners indicate where they want the patient to go. If it is still open, then indeed, it is probably booked wherever there is availability... Because we have not given instructions to TIPP yet. We have not even officially decided yet that arthritis does not belong at the center. After deciding this, we can tell the TIPP employees to keep an eye out for trigger words such as arthritis or active inflammation in the referral letters when handling rheumatology patients. (...)"

GP1 now follows up on M1's solution: "If we give such an instruction to the TIPP employees right now, what is the TIPP employee supposed to do with it? Call the general practitioner to explain that his request is not right?" "No, no, no, no," M1 responds straight away, "a general practitioner either explicitly mentions where the patient should go or leaves it open. In the first case, TIPP does not have to do anything, because then the general practitioner has decided already. I think we should assume that that had been the right decision. In those cases where the general practitioner has not considered the different options, TIPP employees need an instruction, for example that they should filter out the rheumatology patients in whose referral letter arthritis has been mentioned. Then they should tell the patient, 'you are not eligible for the Primary Care Plus center; you should choose a secondary care provider, such as hospital a, b, c or d.' The general practitioner will eventually 
hear what the patient and the TIPP employee have decided together. But if you as a general practitioner then say, 'I thought this patient would be sent to the Primary Care Plus center', then we can refer to the protocol and say, 'In the letter you mentioned arthritis, and this is what we have agreed about that'”.

At this point, the rheumatologist, who had not said anything for a while, joins in the discussion again: "So, we need referral protocols, because we are far from how you depict it. Currently, I see the whole range [of medical problems] here [at the center]." M1: "Yes! That is why I think we need to be doing that right now." The rheumatologist, now reluctantly convinced: "You know, we can make a beautiful protocol together, but still, people make mistakes. But if we have tried our best, then everybody has shown good intentions, and then, well, if a mistake is made, we can accept it as part of being human.”

In the previous excerpt, I argued, GP2's disentanglement and M1's appropriation resulted in two parallel but different and incompatible processes pertaining to what is going wrong in the future and what had gone wrong in the past. In the two parts of the above excerpt, in contrast, the process of knowledge work conducted is oriented to the future instead, enabling a more collective process, more inclusive towards the perceptions and worries of the different participating professionals.

In the first part, M1 imagines out loud how his solution would work out in future situations. He thus entangles the elements of the solution he has proposed, i.e. the instructions for TIPP personal, by imagining different future contexts in which these instructions would or would not be followed. Imagination, Coeckelbergh and Mesman (2007) argue, plays a key part in processes of moral reasoning, because, among other functions, it helps people to project scenarios and in the process of putting themselves in somebody else's place. It thus enables improvisation, tuning and creating new options for taking action. Here, in M1's acts of knowledge work, step by step imagining how the indication lists would work out in the context of TIPP employees making appointments with patients in the future, imagination has similar effects, albeit on a more practical than moral sense. M1 projects how the solution would work out in different scenarios: one in which the general practitioner has given an explicit instruction in the referral letter, and one in case the general practitioner has left it open. Also, in the imagined future work processes, M1 emphasizes that when general practitioners have given an instruction about where the patient should go to, TIPP employees should not do anything. With this small but significant part of the knowledge work, M1 reestablishes the hierarchical relation between general practitioners and TIPP employees, showing sensitivity to the general practitioners' worry that TIPP employees can overrule their decisions. 
In the process of imagining how the solution may work out, M1 places himself in the general practitioners' position. Imagination gives him space to improvise and tune his solution to their worries. Finally, the referral protocol helps shape a new option for taking action: If a general practitioner afterwards does not agree with the decision a TIPP employee rightly made, the indication lists can serve as a contract, an impartial judge, showing that the underqualified TIPP employees nevertheless followed the instructions the general practitioners had collectively agreed to.

The improvisational solo that $\mathrm{M} 1$ performs as part of his knowledge acts of imagination is performative. In the process, he creates a joint to which the three problem realities (system, knowledge deficiency and qualification) can easily connect. When he recounts the details of the imagined procedure, he chooses to do so in terms of arthritis. Applying the solution to the medical problem of arthritis resonates with the effective anecdote of the rheumatologist at the beginning of this discussion, which, throughout the whole discussion, functions as the entry point to the problem. With this knowledge move, the problem realities might not be fully aligned, but they are being connected to a joint, the medical problem of arthritis, and as such are sufficiently dealt with for the organizational processes to start flowing again.

The second part of this excerpt is the knowledge work of the rheumatologist. Her response, stating that the current situation is far from how $\mathrm{M} 1$ describes the renewed procedure, is her way of connecting to the joint created by M1's knowledge work of imagination. Note that this act does not involve a full but merely a partial but sufficient reconciliation of her and M1's and GP2's problem realities. She now agrees to the plan, but not because she believes it will be a solution in her problem reality. At this point, by emphasizing the importance of good intentions, she dodges a reconciliation of content while offering one of procedure. This we can consider a third type of knowledge work conducted at the meeting. It is not an act of reformulation through appropriating elements of incompatible problem realities, neither an act of imaginary entanglement, but a shift from content to process, i.e. from the problem reality and solution to the procedural aspects of bringing the solution into practice.

\section{Understanding relevant referrals in context}

Now having the rheumatologist on his side, the director goes into more detail about how to proceed: "I think if we add more general practitioners to the users' council, they can become part of the teams that will compose these protocols, monitor and consult their colleagues about it. I mean, they [the referral protocols] need to be composed in dialogue with the primary care world, in which doctors can have very different motives for referral than those that are apparent to us. How relevant a referral is depends to a large extent on the lens through which you are looking at it 
[the problem]. In the past, when we held joint consultations 30 , the involved rheumatologist considered $80-90 \%$ of the referrals to the joint consultations relevant. But when he saw patients on his own at the hospital, he considered $80-90 \%$ of the referrals from the same randomized group, coming from the same general practitioner, from the same population, irrelevant, because in the hospital he would have [only the referral letter but] no information about the context in which the general practitioner had made the referral decision."

The rheumatologist jumps in to add a similar experience: (...) "When our waiting lists for appointments at the hospital were extremely long, we started calling the general practitioners that had referred the patients. Often, referrals are right, but sometimes there is obviously nothing wrong with a patient. We would call and ask, what do you want us to do with this patient? The message was often 'listen, I don't know what to do anymore. I know you don't know either, but this patient is driving me crazy. She comes to the practice every week. Please, help me out here.' A few years back, one of these patients was truly terrible. I agreed with the general practitioner that she could have an appointment in the hospital in six months. This enabled the general practitioner to tell her she was on the waiting list and to keep her away from the general practice for a while. We had come up with this plan together. We thought it was a good idea, but yes, only because I knew the context. It often happens that I make the same diagnosis as the general practitioner, but I can feel that the patient had not accepted it before. It would be very nice if such patients could be sent to the Primary Care Plus center." (...)

Director: "This is exactly what could be pleasant about offering specialist care through this facility. And that is why I say, you [addressing specialists here] cannot unilaterally compose such lists and implement them. It is the dialogue, from which we can learn how it should be implemented and how it should work in detail. What signals should we [general practitioners] give to clarify what we want and what we expect from a Primary Care Plus consultation. Right now, we are sitting here with only two general practitioners. We need a group instead. One of us will team up with you [addressing the rheumatologist], one with you [addressing the neurologist ] and one with you [addressing the orthopedist]. (...) If we keep improving the selection at the front door, (...) sooner or later you will be able to have trust in getting the right patients [in your Primary Care Plus consultation hours].”

This excerpt shows another phase of knowledge work. In the previous excerpt, sufficient reconciliation was achieved between the director and the rheumatologist,

30 The director refers here to his $\mathrm{PhD}$ research, in which he studied joint consultations between rheumatologists and general practitioners. 
with knowledge work mainly directed at the solution offered by the director. The knowledge work conducted in this excerpt, in contrast, is mostly directed at the problem realities. At the beginning of the excerpt, M1 brings in a new perspective in the discussion: the problem is not the incorrectness of referrals but the fact that the medical specialists misunderstand them because of a lack of feeling for the context. Composing referral protocols is thus not only of value for its result, but also for a procedural aspect: the "dialogue" with the "primary care world".

The above knowledge act has two performative effects. First, M1 reshapes his problem reality from a lack of knowledge on the side of general practitioners and TIPP employees to the disconnection of medical specialists from the general practitioners' reality. The problem is transformed from one of knowledge deficiency into a problem of irreconcilable worlds. Secondly, another norm for good referral is produced. In this knowledge work, good referral is not a matter of deducing the right decision from the collected medical information but a situated practice that can only be assessed by taking the specifics of the context into account.

For the rheumatologist, M1's reshaped problem reality resonates with a past experience when she also needed to know what the general practitioner had experienced with a patient to understand and support a general practitioner's referral decision. Even though M1's claim and SP1's experience imply a different role of contextual knowledge in understanding a referral - in M1's case, contextual knowledge is needed to explain a good referral practice; in SP1's case, the contextual knowledge explains why a general practitioner made an incorrect referral - it again forms an important joint for finding sufficient reconciliation. The referral protocols are now seen as a broad and effective solution, not only because they are a quick way to provide information, but also for its processual effect of connecting the primary and secondary care world. Again, what this shows is that knowledge work is not a matter of thoroughly aligning disparate problem realities, i.e. of creating consistent worlds, but of merely connecting experiences and convictions at the joints. Even though M1's and SP1's knowledge actions produce incompatible norms for good referral, they now endorse the same solution. What it also shows is that more than performing a "play of resistance and accommodation", as Pickering (1995, p. 23) characterizes the mangle of practice, the professionals present at the meeting are actively seeking to establish 'joints of agreement' in order to be able to further collaborate.

Bringing in the problem of contextuality and the rheumatologist's enthusiastic connection to this knowledge activity also has an important normative effect. In the exchange, the director and the rheumatologist now have firmly established a much broader norm about what good referral entails. A good referral practice does not only depend on the medical aspects of the patient's problem but also its social 
dimensions, such as how well the patient accepts the general practitioners authority and whether seeing a medical specialist might be an effective way to keep the patient from weekly consulting the general practitioner; a referral can be justified, even when medical grounds are lacking. Moreover, whereas in earlier problem realities, the specialist's decision was the indication of the rightness of a general practitioner's referral, implicating an hierarchical knowledge relation between medical specialist and general practitioner, these knowledge activities level the ground: Knowledge of context and motivations of general practitioners and the diagnosis of a specialist are equally important for understanding and justifying referral practices.

\section{Beautifully repetitive referral patterns}

Right before the discussion goes into other topics, the rheumatologist interrupts: "This afternoon, we had a very interesting presentation of somebody who had studied our referral letters.31 It was wonderful. She had found beautifully repetitive referral patterns. The conclusion was that about $50 \%$ of the referrals [of general practitioners] to the rheumatology outpatient clinic are not necessary!" GP1 responds full of surprise: "Half of the referrals? Maybe we can take some interesting insights of that research!" SP1: "The unnecessary half consisted of patients with, for example, fibromyalgia, osteoporosis, and - I have to say I am proud of this - only $5 \%$ of these did not receive a diagnosis." GP1 is still pondering on the news: " $50 \%$ of your patients might as well have not been referred to secondary care...? That is a huge amount! (...) Do you happen to know how many patients have appointments at your outpatient clinic yearly?" SP1: "Around 3500." M1 joins in the discussion: "We are talking here about 1700 unnecessary referrals! To prevent these referrals, we need to organize a smart way of triaging the patients." GP1: "We are talking about a lot of money here. That is great!" M2 shares in the enthusiasm: "Finding how to take these out, is what our project is all about here!" (...) SP1: "Apparently, this is really complicated. But if we succeed to take only a quarter out, it is already an accomplishment." M1: "Yes, yes, of course. That is what it is all about!" A: "We need to carefully describe this group of unnecessary referrals."

In the above excerpt, the reconciliation process is concluded. Now, the professionals, who were reluctant at first, not merely agree but enthusiastically endorse the research as a proof of composing indication lists to be a feasible solution. With this data in hand, indication lists transform from a solution to an organizational problem into a direct and important contribution to the actual mission of the overall project:

31 The rheumatologist refers to the letters of the medical specialists working at the hospital have written after a consultation with a patient. 
making specialist care more efficient, that is, filtering out the medical problems for which specialist care in the hospital is unnecessary.

This excerpt also brings forth the first conclusion of this subsection: Integrated-care-in-action is not a linear but a diamond shaped process - in this case - consisting of three phases. In the course of the knowledge work, aspects of the knowledge practice of managing Primary Care Plus, such as the indication list, but also the norms about what good care entails and the knowledge relations between different medical professionals, were not given but were shaped in the process of knowledge work.

In the first phase, the start of the discussion, the suggested problem-solution fit produced a narrow understanding of the problem (knowledge deficiency on the side of the general practitioners) and appropriate solution (list with the information that the general practitioners are lacking). In this phase, the indication list was a carrier of information about suitable and unsuitable medical problems for Primary Care Plus, which would enable an unproblematic knowledge transfer from medical specialist to general practitioners. Relatedly, good referral was seen as an orderly and linear process in which a general practitioner takes the right decision on the basis of medical information she gathers about the patient's signs and symptoms. Note that in this phase, a specific hierarchical relation between general practitioner and medical specialist was produced: By proposing the transferal of medical specialist knowledge to the general practitioners as a solution to the problem, it was assumed that specialists possess more knowledge about specific medical problems than general practitioners.

In the middle phase, it became apparent that the fit between problem and solution was not a given. In order to achieve the fit, knowledge work was required: Problem and solution realities were first tweaked and broadened through appropriation, imagined entanglement and shifting from content to process. Sufficient reconciliation was achieved, not by full alignment or integration but by creating effective 'joints of agreement'. In this rich process of knowledge work, the indication list acquired a variety of meanings: From a document not only informative for general practitioners but also for TIPP employees, it became a "filter", a set of "trigger words" that would function as an algorithm, helping underqualified TIPP employees without referral skills or knowledge to take the right decisions; a contract that would come in handy to remind general practitioners of what they agreed on; and lastly, a process in which collaborating general practitioners and specialists would create mutual understanding. During the middle phase, different norms of good referral emerged. In the solution reality produced by algorithmic understanding of the list, good referral became a skill of general practitioners developed through the right 
education and experience. With the indication list as a process, good referral became associated with the specific (social) context of the patient as well. The different realities of the indication list, the related set of norms about good referral, came with a shift in the knowledge relation between general practitioner and medical specialist; now, the general practitioner became a crucial source of contextual knowledge, indispensable for understanding the relevancy of a referral.

In the last phase, after reconciliation was reached, the problem reality and appropriate solution, now a sufficient fit, were narrowed down again, dismissing the knowledge and meanings gained in the previous phase of knowledge work. Indication lists, based on letters that rheumatologists have sent to general practitioners after diagnosis, are presented as the solution. They will carry medical knowledge about the specific medical specialty to the general practitioners whose faulty referrals are caused by knowledge deficiency.

The second conclusion that I draw from the above analysis is that integrated-care-in-action is primarily concerned with dealing with knowledge differences by creating joints of agreement. Sufficient reconciliation was achieved by connecting the different problem and solution realities to the joint of arthritis, which played the leading part in both the knowledge work of imagination and the sufficient agreement that SP1 found in these steps. This is in contrast with the Integrated Care literature I referred to above, in which Integrated Care, and its knowledge benefits, are seen as an effect of "crossing boundaries" or "cutting across multiple services, providers and settings". Changing the metaphor, from one of boundaries (a geographical/political metaphor) to one of joints (anatomy metaphor), comes with implications. When viewing the dynamic practices of managing Primary Care Plus, joints connecting diverse and incompatible worlds might be more productive for seeing and understanding what integrated-care-in-action entails than taking the work as a process of leveling the ground, i.e. taking out the fence (integration).

The third conclusion is that the knowledge work of integrated-care-in-action does not start and end in one place in one time slot; it unfolds in a sequence of events. So far, in this chapter, I have analyzed two discussions related to the indication lists emerging as a key tool for managing the delivery of Primary Care Plus. In this process of emergence, at least three events can be identified in which professionals conducted knowledge work concerned with embedding the indication lists in organizational processes. In chronological order, the first event is the discussion taking place early 2015 at the Primary Care Plus center in the West side of the city, analyzed in this section, in which the indication lists came up as a possible but contested solution to the problem of unsuitable referrals. After the knowledge work required for achieving a problem-solution fit, the second event would then be 
the efforts of the teams of general practitioner and medical specialist to compose such lists, taking place after the analyzed users' council meeting - not analyzed in this chapter. The third is the presentation and the discussion, of which I analyzed a fragment in the introduction of this chapter. Here, the knowledge work of addressing and accommodating conflicting meanings was needed to make the list function as desired, i.e. to start embedding the list in the daily work processes of general practitioners. The dynamic process of integrated-care-in-action, i.e. the knowledge work involved in making collaboration run smoothly, was thus distributed over a sequence of events. Further drawing on Rouse's (2001) work, presented earlier in the chapter, indication lists are like models. They are not representative of a stable reality out there in and of themselves, i.e. they are not the right solution to an unambiguous problem waiting to be discovered; they become an effective solution by taking them up in "ongoing patterns" (Rouse, 2001, p. 203) of appropriation, disentanglement, imaginative entanglement and shifting from content to process, in which connections are build that render the events into a sequence of knowledge work.

\section{ALIGNING KNOWLEDGE REALITIES}

In contrast to Primary Care Plus' promise of learning through health care integration, I have shown in the previous subsection that other kinds of knowledge activity are prominent in a practice of integrated care. Integrated-care-in-action is the process of aligning problem realities sufficiently to start the collaborative work of bringing a solution into practice. I have drawn three conclusions about how integrated-care-in-action takes shape in the knowledge practice of managing the delivery of Primary Care Plus. First of all, I have argued that it is not a linear process flowing naturally from a design but a diamond shaped one, in which different aspects such as the indication list, norms of what good referral entails and knowledge relations between different medical professionals are continuously shaped and reshaped. Secondly, integrated-care-in-action should not be framed in terms of "cutting services" or "crossing boundaries" but in terms of building joints of agreement. Such a shift in metaphor gives space to understand how collaboration is possible without striving for (full) integration of differing knowledge worlds. Thirdly, analyzing two discussions related to indication lists, I have argued that integrated-care-in-action is a sequential process in which elements, such as the indication lists, are made to be self-evident solutions to certain problems. Departing from integrated-care-in-action as a sequential, non-linear process of creating joints of agreement in this subsection, in this section, I will shift focus to knowledge work as an activity of actors who follow discernable logics. The concept of knowledge that I introduce here in order 
to show how the different approaches of knowledge work internally cohere more or less, refers to the way in which a set of actions is orchestrated as a consequence of implied norms prescribing what counts as knowledge and what not, and how knowledge differences should be dealt with. In the course of the chapter, I identify three general logics, each with a different aim: integration, connection and accommodation.

At the time of the users' council meeting, discussed in this findings' subsection ${ }^{32}$, the centers were running for around ten months. The numbers of patients treated at the center were not as high as projected in the business plan written before the centers opened their doors. The managers of the project were constantly exploring opportunities for expansion of the consultation hours with new specialties in order to get the patient numbers up. When the ethnographic work started, patients could see a specialist for internal medicine, an orthopedist, an eye doctor, a neurologist, an ENT doctor, a rheumatologist and a dermatologist at the center. A year later, gynecology, urology, sexology, pediatrics, and CVA nursing were added to the options. When expansion plans were in the making, they would be extensively discussed with the general practitioners of the users' council meeting. 'Expansion plans', a solution to the problem of low patient numbers, had become a permanent item on the agenda.

The meeting discussed in this findings section took place four months after the meeting of the previous subsection. In these four months, at least four new general practitioners had taken a seat in the council. As a result, the spring meeting was attended not by two general practitioners, but by six (GP1, .., GP6). Because the newly hired daily manager (M3) was still settling in, the project manager (M2) of the hospital, taking care of practical issues before the new manager was hired, attended the meeting as well. Before this meeting, management had decided that it was not necessary for medical specialties of the center to be represented at every meeting. From the handful that were invited, only one, a rheumatologist, was able to attend. Furthermore, there were four researchers from the university research team present. The rheumatologist and the researchers did not contribute to the discussion analyzed in this section.

I start the analysis at the point in the meeting where the tension between knowledge acts become discernible. Then, I will trace the knowledge work conducted to sufficiently reconcile the emerged differences in order to enable proceeding with the organizational work.

32 Meeting room, June 11, 2015, 5:30-7.00 PM 


\section{Expanding the center with spine consultation hours}

After giving a short overview of the upcoming appointments with hospital departments, M2 presents the more advanced plans to expand the centers' services with special spine hours: "The spine center [at the hospital] offers multidisciplinary consultation hours with orthopedics, neurology, rehabilitation medicine and doctors specialized in pain treatment. (...) At the moment, it is not possible [for general practitioners] to directly send patients to the center. (...) the preselection takes place at the hospital departments [orthopedics, rheumatology and neurology] where general practitioners usually refer such patients to. You know, those instances where a patient or a general practitioner feels the need for a specialist taking a quick glance as well... (...) Because only ten percent of them will get a real treatment, it would be better to do the preselection outside of the hospital. There is no need for these patients to be sent to secondary care. We are talking here about 1100 patients getting such a DBC [lower back pain].”

After hearing the number 1100, one of the present general practitioners, GP1, makes a puzzled face. "Where are these patients coming from?" he asks in disbelief. (...) M2: "From the general practitioners." GP1: "Yes, but which general practitioners?" Another general practitioner, GP2, jumps in: "Spine center patients... they come from the whole province!" M2, somewhat agitated: "That is not what I meant... I am talking here about patients with lower back problems. There are 1100 patients per year [from this region with lower back problems] of whom hardly anyone ends up at the spine center. Now, they end up at the neurologist, the orthopedist [at the outpatient clinics of the hospital]... These specialists look at it and then make the conservative decision to keep them in primary care. (...) From there, the specialist can decide to start primary care trajectories, such as physiotherapy or psychology, or she can decide to send the patient to a social worker. But... [these decisions are then made] with the specialized knowledge of the spine center. (...)" Another general practitioner present at the meeting, GP3, now asks: "So what is different from the current situation then? We could also send such patients to a [normal] orthopedist in the center, couldn't we?" (...)

This excerpt marks an informative point of departure for tracing knowledge work. The manager's knowledge actions produce a knowledge reality, a self-evidently fitting constellation of problem (in this case low patient numbers), solution (expansion of the center) and justification (1100 referrals of patients with back problems) that emerges as a consequence of the conducted knowledge work. Situated within M2's actions, the number 1100 marks an opportunity ready to be seized by setting up spine center consultation hours. It is an indication of a large group of patients 
that could potentially be brought in as Primary Care Plus patients.

GP1 and GP2's negative response to the presentation of the plans show that within their knowledge realities, the number 1100 does not count as an appropriate and self-evident justification of the expansion. In the manager's knowledge reality, it is a simple puzzle of three pieces forming one straightforward image; whereas, within the knowledge reality of GP1, GP2 and GP3, the number of 1100 is first and foremost a source of confusion. If anything, it indicates that colleagues are making problematic referral decisions. Puzzlement, expressed in words and through body language, has a normative effect: It redirects the attention from the center, responsible for solving such a source of redundant secondary care referral, to the general practitioners themselves who are making the redundant decisions in the first place. In GP3's knowledge actions, the pieces of the puzzle fit neither. He acknowledges the problem but does not see a reason for adding another specialty at the center. The orthopedist or the neurologist who then had consultation hours at the center might as well see those patients. If it were up to him, there is no need for starting a brand new spine consultation hour. With three clashing knowledge realities, the one of M2, the one of GP1 and GP2, and the one of GP3, knowledge work is required before decisions about the expansion plans can be made.

\section{What kind of orthopedist care is suitable?}

M2 responds to GP3's skepticism: "It might be easier to communicate with other specialists here [than at the hospital]. Basically, the plan is to instruct general practitioners to refer all patients with back problems - except of course when the problems are related to oncological problems and secondary care is evidently needed - first to the Primary Care Plus center. In this way, we use specialist knowledge in a primary care setting for selecting patients that actually need to go to the spine center in the hospital. We estimate this will amount to $10 \%$ of those 1100 patients who are being referred to the hospital now." After M2's answer, GP3 asks another question: "Do they [the patients] need to fill in one of those questionnaires?" M3 answers confirmative. M2: "They stay in primary care. That is the core idea. And in such a primary care setting they [the specialists] are very well able to further...”.

After M2's unfinished sentence, a short silence follows in which a fourth general practitioner, GP4, starts expressing her hesitation about the plan as well: "what is currently bothering me somewhat is that those patients, the 1100 , with chronical back aches, we send them to the orthopedist or to the neurologist, or to the pain center..." "Or to the rheumatologist," the second general practitioner, GP2, adds. GP3: "Right now, they are examined with a mono-disciplinary view. The orthopedist, or $u h m$... the specialist now needs to think, 'is this one for the spine center? 
Is there more going on than just lower back issues...?' If I understand it correctly, when we [general practitioners] see patients with chronical lower back, and some other diagnoses, we should send them to the Primary Care Plus spine center (...). There, a broader view is taken up than just a neurological or orthopedist view...?" M2: "Well, in the center, in first instance, orthopedists see the patients..." GP3: "So it is the orthopedists who examine the patient, but the questionnaire that is used to determine whether there are also psychosocial problems is extra?" M2: "Yes. And possibly we can organize a neurologist to hold parallel consultations. We can imagine that they [orthopedist and neurologist] then would consult each other..." At this point several general practitioners start talking. One of them, GP3, says, "Well, then we should return to the basic question: What kinds of orthopedist care is suitable for the Primary Care Plus center and what is not?" M2 responds: "Yes, and this is actually one [type of suitable orthopedist care] that is easy to communicate [to the general practitioners].”

In the second excerpt, three instances of knowledge work aimed at aligning the disparate knowledge realities can be discerned. Analyzing the first excerpt from this subsection, I argued that for the three general practitioners, M2's fit between problem, solution and justification is not a given. The first instance of knowledge work in the above excerpt is M2's reconfiguration of her knowledge reality, as a response to GP3's question into the added value of a Primary Care Plus spine center. In the new constellation, not the low patient numbers but the fact that 1000 of the 1100 patients are redundantly sent to the hospital is now presented as the problem that needs to be solved. The added value of sending the patient to the spine center specialist in comparison to the specialists already working there becomes the justification: The spine center consultants can bring the needed knowledge to the Primary Care Plus center to make the right decision and holding spine center consultation hours at the center will offer the opportunity for specialists to collaborate. The new configuration is tailored to fit the knowledge reality of GP3, for whom the added value of the plan is a crucial aspect.

The second instance of knowledge work aimed at aligning the knowledge realities is a collective effort of the present general practitioners and the manager to disentangle the current referral scenario and imagine the proposed one in order to bring the added value of the new plans into view. GP4 starts off this instance of knowledge work by disentangling the variety of considerations of general practitioners in the current scenario: A general practitioner has a specific idea about whether to send a patient with lower back problems "to the orthopedist or to the neurologist or 
the pain center." Subsequently, GP3 and GP2 add other aspects that are missing in GP4's act of disentanglement. Halfway, GP3 shifts to projecting the proposed procedure, pointing out a crucial difference along the way: the possibility of a "broader view" instead of a "mono-disciplinary view". Finally, M2 makes other additions explicit: a "questionnaire" addressing "psychosocial problems" and possibly "parallel consultations" with the neurologist. Through disentangling the current one, projecting the proposed one and contrasting the two scenarios, they collectively answer GP3's question regarding the added value of the spine center plans.

As a response to the collective effort to map more and more items of added value, $\mathrm{C}$ sets out a third act of knowledge work. This one is quite common in the users' council meetings. By asking the "basic question into what Primary Care Plus orthopedist care" entails, he reminds the others of the initial purpose and the identity of the center. With this act, he ends a process of bringing in new elements that answer the question about the added value. By adding more and more extras, instead of supporting the goal of reducing costs, the project could end up becoming more and more complex, expensive and time consuming. By reminding the present general practitioners and managers of the initial purpose, he tries to avoid entering a process in which unneeded extras are being created.

\section{Relating to the number 1100}

At this point, one of the general practitioners, GP4, returns to the number 1100 again: "I am surprised about the number 1100, because I think..." GP2 interrupts - now even more decisively: "No, those 1100 patients, they are not coming from us!" M2 starts addressing GP2's concern directly: “You know what it is, GP2, these numbers are based on the DBCs. On the code that the specialist notes down after all the examinations have been done. It is hard for you to relate to this number, because you [as general practitioners] mainly refer patients on the basis of signs and symptoms, not on the basis of diagnoses. And if your patient does not feel helped by you, she will go to Belgium, she will start 'shopping'. These patients who have lower back aches, they..." GP2 interrupts, again firmly: "I do not refer these kinds of patients to an orthopedist." GP3: "If I look at my own practice and if I think about what I do there, I rarely..." M2: "What you have not been doing until now, you should not start doing of course. (...)"

Another general practitioner, GP5, who has not said much until now, adds to the discussion: "It is a good idea to look at these patients with a multidisciplinary view. The orthopedist... a questionnaire... I think... well... the integratedness of the plans... I think that is really good." Then GP4 says: “(...) If you view these problems from the orthopedist's perspective, nothing is wrong with such a back. 
But that does not work for the patient. It is smart to broaden it." M2: "Yes, and that is not something we should be doing in secondary care. They [the initiators of the expansion plan] think only $10 \%$ of those 1100 will get a treatment. Thus, for 1000 patients, the hospital visit was not needed if there had been more knowledge [at an earlier stage of the trajectory]. It is only because otherwise the patient goes 'shopping' or the general practitioner thinks, 'you know what, I will just refer this patient."

In this excerpt, it is mainly M2's knowledge work that stands out. In the beginning of the excerpt, GP4 brings up the number 1100 again. While for the manager it is a self-evident indication of an opportunity to be seized (justification) or a problem to be solved, for GP2, who interferes in this part of the discussion as well, it is a source of confusion: He does not understand, nor believes, that this many patients with lower back problems are redundantly referred to secondary care. In the previous excerpt, M2 attempted to reconcile this difference by broadening the added value of spine center care as Primary Care Plus care, but now, she addresses the difference from an outsider position. She explains that the meaning of the number as she initially used it can only be understood departing from the specialist's practice, whose actions are aimed at determining diagnoses, and not from that of the general practitioner, who focuses on gathering the signs and symptoms of a patient. While the number indicates that specialists have given 1100 patients the diagnosis of lower back pain, the general practitioners might have indicated other, less clear signs and symptoms. Instead of trying to make the knowledge differences compatible as she did in the above excerpt, she now sets out to create mutual understanding by explicating different knowledge practices. As such, she does not aim at aligning or integrating knowledge differences but to turn the meeting into a joint between differing knowledge practices. This has a performative effect: Earlier, the professionals used the DBC of lower back pain, which the specialist gives to patients during his consultation to make sense of why and how a patient had come to the hospital; in this instance of knowledge work, she makes space for how such patients are assessed within the knowledge practice of the general practitioners, making space for a broader palette of considerations.

While GP1 dismisses the opening enacted by M2 - he still thinks these patients should have never been referred in the first place - GP4 now joins in the knowledge work of explicating the different professional knowledge practices. In switching back and forth between knowledge practices, they create a new world together: one in which patients are not either necessarily or unnecessarily referred but in which there is an intermediate kind of referral - patients for whom specialist care 
might seem redundant from the "orthopedist's perspective" but for whom some specialized knowledge and a broader approach is valuable nevertheless. The expansion plans make it possible to help such patients outside of the hospital. Within this world, incompatible considerations from both practices are taken into account while keeping the gate of the hospital closed. By switching from world to world, the two managers have shifted from aiming to achieve a coherent, unified world (integration) to aiming to create a connection in a fragmented one (connection).

\section{Putting the plan on paper to discuss it with colleagues}

After the disparate responses, one of the general practitioners, GP3, now wonders out loud: "What are we going to do now [with these plans]?" M1: "I take from this discussion that you are saying: 'No, we will not give a "go.'” GP1, with a somewhat affable but condescending tone of voice: "No. I think it is too vague." GP2: "I wonder what the added value would be..."

GP6, one of the general practitioners who had not added anything to the discussion yet: "What about, if we receive a plan on paper and we use that to discuss it with our colleagues?" GP3: "I think that is a good idea." GP2 again repeating his position: "Do we really need another possibility for referring our patients with lower back problems?" GP3: "I would just send these to the center. If you happen to have a difficult patient who pushes to be sent to a specialist, that is what I would do." M1: "Well, if you look at the current volumes [of patients with lower back problems], and each of those patients' general practitioners would do that, then placing a specialized orthopedist there to see these patients is justified."

After GP3 shared some thoughts out loud, showing a growing willingness to consider the plan to be fruitful, M1 now starts concluding the discussion. M1: "So we could decide that in principal, all lower back problems would be sent to these specialized consultation hours, unless secondary care is obviously needed. This is what the initiators propose, 'Just send these patients for a quick advice to the center first, that could be sufficient already.' The added value is not only a quick 10-minute consultation in which the specialist decides whether or not the patient is qualified for the actual spine center, but there will be some more extensive support for these patients." GP4: "For me, this really adds something." M2: "Ok. I will send the plan around and we will discuss it further."

In the first three excepts, I showed two logics of knowledge: the efforts to fully integrate knowledge realities and then, the attempts to reconcile different knowledge worlds by connection through joints. In this fourth excerpt, a third logic of knowledge work can be identified. The opinions about the plan are still diverse: GP1 thinks 
the plan is "too vague", GP2 is not convinced about the "added value". Others are more willing but still hesitant. These different reconfigurations of the fit are not the same for everyone, but they are sufficiently in line to agree on taking the next steps. The final act of knowledge work, however, is the act of relocating the discussion to another space and time and thus, involving other people in a similar position. At this point in the center's existence, around six general practitioners have a seat in the council. These general practitioners are not elected to function as representatives, they do not have decision making power, but are seen as a source of knowledge about the general practitioners' perspective to the management process. The center provides Primary Care Plus not for these six but for ninety general practitioners in the region, whom a general practitioner during an interview characterized as a "bucket full of croaking frogs" when it comes to policy issues. For the general practitioners to not unanimously agree on a plan is no reason to refrain from executing the plan. "Putting the plan on paper and discussing it with their colleagues", in this trajectory, is an act of knowledge work that follows another logic, one that aims at consolidation through accommodating the decision making elsewhere. This is the logic of distribution.

Earlier, I have shown how an - according to some - inappropriate justification of the expansion plan that an orthopedist had proposed, the fact that 1100 patients were wrongly referred to the hospital, produced friction, which was followed by a process of knowledge work that consisted of different acts. First, I noted knowledge work that, through reconfiguration, (dis)entanglement and boundary setting, aimed at making the different knowledge realities compatible, at fitting the different understandings of the problem, solution and justification in one coherent picture. Interestingly, the initial misunderstanding between the different professionals was related to the meaning that each of them attributed to the number of 1100 patients with lower back problems sent to the hospital. For the manager, it indicated a clear opportunity to be seized; for one general practitioner, it was a source of confusion; for another, evidence of the problematic referral behavior of general practitioners. The strategies were aimed at aligning these differences of meaning, while the number itself, 1100 patients referred with lower back pain, was taken as a given, as an unproblematic representation of a state of the world. The set of knowledge work activities thus also produced a hierarchical knowledge relation between specialist and general practitioner: The DBCs that the specialist had given to patients was used as a standard to assess the general practitioners' referral decisions.

Then, by switching from one knowledge world to the other, from the consultation room at the outpatient clinic, where doctors give DBCs to patients, to the general practice, where the doctor bases her treatment decision on signs and symptoms, 
the conducted knowledge work was aimed at connecting worlds through joints. In this instance of knowledge work, the number is not taken as a given anymore. Its self-evidence is countered by questioning its production process. As such, the manager brings in some interpretative flexibility, allowing for plural knowledge worlds to coexist, and thus, for a more horizontal knowledge relation between the general practitioners and medical specialists. These knowledge work acts create space for the world to be incoherent. Agreement is not sought in creating one integrated world but in accepting its incoherence.

Finally, consolidation of the plans is sought by distributing the heterogeneous knowledge realities. By postponing and relocating the decision, differences are not resolved but lodged elsewhere. Knowledge work, as a crucial aspect of integrated-care-in-action, thus not only consists of different kinds of actions (entanglement, boundary setting and reconfiguration), but it also follows certain discernable epistemic logics of - in this case - integration, connection and distribution.

Note that the notion of "logic" that I have used here can now be further specified. The manager, for example, in the process of presenting a new idea and responding to objections, went from seeking to make knowledge realities compatible to switching from one knowledge practice (orthopedics) to the other (general practice). The way I use the notion here is inspired by Hacking's (2002) notion of styles of (scientific) reasoning. As much as "reasoning", according to Hacking, "is done in public as well as in private: by thinking, yes, but also by talking and arguing and showing", logics, in this context, are thus not characteristics of a process of reasoning taking place in isolation in the mind; they are shaped in and by social interactions. ${ }^{33}$ Also, logics are plural. Knowledge actors often switch from the one to the other in the course of collectively conducting knowledge work.

\section{CONCLUSION}

In marketing material, reporting and mission documents, Primary Care Plus is presented as a promising new way of organizing the delivery of specialist health care. One of the most important claims in the writing and speaking about Primary Care Plus is that general practitioners will get the opportunity to learn from the Primary Care Plus medical specialist they refer their patients to and thus improve their gatekeeping skills. In the previous chapter, I have argued that these claims fit the trend in health care to take integration of health care services as a key strategy for improving quality and cost-efficiency of care. More specifically, it is akin to the prominent claim within Integrated Care literature that medical professionals involved in

33 This resonates with Mol's $(2008$, p. 8) conception of logic as rationales by which practices "hang together" as well. As I use the notion logic as embedded in social interactions, Mol's logics are not always "verbally available" to practitioners; they are implied in practices. 
Integrated Care initiatives extend and broaden their medical knowledge. I pointed out that such an understanding of the knowledge dimensions of Primary Care Plus as Integrated Care is set in Pickering's representational idiom, because it assumes that medical knowledge is a potentially coherent representation of the human body in all its aspects.

Tracking the knowledge work of integrated-care-in-action, i.e. the efforts to deal with knowledge differences in order to enable organization processes to run smoothly, showed that the representational idiom of Primary Care Plus promises gives a narrow and limited understanding of its knowledge dimensions. The knowledge practices of managing the delivery of Primary Care Plus is a rich and dynamic process that does not flow automatically from its design. The present professionals conduct a variety of knowledge work acts in order to enable organizational processes to start running fluently again. They disentangle and explicate problem realities to subsequently entangle them in an imagined solution reality. They rearrange their problem realities by appropriating parts of others, shift from content to procedure of solution realties. They reconfigure knowledge realities, they recall original identity and purpose of the project, and they build joints by shifting from perspective to perspective and distribute differences by relocating the discussion.

In tracking and dissecting these knowledge work acts, I drew two broad conclusions. One pertains to knowledge work as a process unfolding in practice and one to categorizing the diversity of actions through discerning their epistemic logics. First, the process of knowledge work is sequential; it consists of a sequence of events taking place in different settings at different times that are connected through the problems and solutions discussed. Such processes are not linear, nor predictable or cumulative. The processes of knowledge work unfolding in practice involve gains and losses of knowledge, meanings and understanding.

The second broad conclusion is that while conducting knowledge work, knowledge actors attune to more or less coherent sets of norms about knowledge that are not very dissimilar from approaches in epistemology: realism (integration), contextualism (connection) and pragmatism (distribution). Sequences of knowledge work actions of professionals involved in the discussion follow a certain logic, the steps and norms they produce and reproduce fit a certain view of knowledge. The logic of integration conveys a representational view in which professionals aim at agreeing on one unified body of knowledge that represents the world out there (even if it is an untenable ideal on the background). When following the logic of contextualism, knowledge actors allow for heterogeneity while reaching for points of connection and by distributing conflicts. As such, it is not the agreement on knowledge that has priority but for the organizational processes to proceed. Professionals, however, are 
not systematic and coherent thinkers such as epistemologists intend to be - in the course of the meeting, they switch from one to the other logic - neither do these logics reside in the mind. The sequences of knowledge work actions are embedded in the social material context, i.e. they are the result of constantly navigating the playing field consisting of emerging material, and social and human agencies. The managers and doctors involved continuously test the water, that is, they feel out resistance and receptivity that emerge after new ideas are presented and points of critique are raised. In that sense, the adopted logics are as much part of the playing field as the professionals themselves, the indication lists, the slideshow presentation projected on a screen, and the tables and chairs in the meeting room. Such logics are not residing or unfolding in the minds of individuals; they are inextricably bound up with the social and material world.

Inspired by Pickering's (1995) performative idiom, in this chapter, I took the practice of managing the delivery of Primary Care Plus as filled with emerging agencies. In this chapter, I mainly focused on the agency implied in knowledge work. In order to understand this type of agency, I borrowed from Strauss' notion of articulation work and Gherardi's (2014) understanding of the practice approach. I argued that there is not only a temporal and mobility dimension to articulation work but also a knowledge dimension. Managing the delivery of Primary Care Plus requires the mundane and often invisible work of somehow sufficiently reconciling the distinct knowledge practices involved. The knowledge work conducted has normative implications and produces relations between professionals involved.

In the next chapter, I shift my focus to the second claim of Primary Care Plus: Relocating specialist care makes specialist care more patient-centered. In order to explore how patient-centeredness takes shape in practice, I move from the meeting room to the consultation room, tracking and dissecting not integrated-care-in-action but the interactions between patient and specialist making diagnoses and treatment decisions in the setting of the Primary Care Plus consultation room. Articulation work, it turns out, does not only have a temporal, spatial and knowledge dimension but also a moral one: In order to achieve durable treatment decisions, articulation work is required to align, accommodate or resolve moral differences. Tracking these articulation work activities will not only give a more in-depth understanding of patient-centeredness but also of how Primary Care Plus is being done in the consultation rooms of the centers. 


\section{4 \\ CRAFTING PATIENT-CENTEREDNESS THE INTELLIGENT ROUTINES OF JUSTIFICATION WORK}

\section{Tear duct as sewage pipe}

The patient seems at ease in the Primary Care Plus consultation room. She sits comfortably on the examination chair, holding her purse on her lap. Before the consultation, she explained that when her eye first started tearing up, her general practitioner prescribed a cream, which she used for thirty days. On holiday in Spain, two months ago, she went to a clinic to see an eye doctor, because the cream had not been effective at all. The doctor in Spain suggested to take a photo to see if and where the tear duct was clogged up and offered to perform a small surgery. The patient decided that if she underwent such a procedure, it would be at home. That is why she did not make a follow-up appointment with the doctor in Spain but, after returning home, asked her general practitioner to refer her to a local eye doctor to discuss the problem further.

Now, she tells this specialist doctor about her eye tearing up and her visit to the Spanish doctor. After the anamnesis, the medical specialist tests the patient's vision and examines her eye with a slit lamp. In the meantime, the patient, her partner and the doctor speak in local dialect and make jokes about the patient's eye. "Are the emotions running high?", the doctor asks the patient when tears roll down her cheek. The patient immediately starts laughing out loud.

Towards the end of the consultation, the specialist presents the treatment options: "There seems to be a thin layer of lachrymal fluid on the retina. But besides that, your eye looks very good. We can inject something into the tear duct and see if that solves the problem. If not, there are two options: either we perform a surgery or we refrain from doing anything at all." With slightly ironic tone of voice, he adds: "A surgery is a serious intervention, we would be chopping, cutting and breaking, as if we are repairing a clogged-up sewer."

Before the consultation, the patient came across as hesitant about surgeries and hospital visits in general, but she was not set against an operation, because the eye was a serious nuisance: "My sight keeps becoming blurry. Every time that happens, I need to get a tissue and press on my eyes. After pressing, the tearing stops for a short while. But then it comes back again.” 
After explaining the first two options to the patient, the doctor asks: "How much does it bother you in your daily life?" Patient: "At the beginning of the day, it is fine. But after a while, it becomes worse." Specialist: "We can try if the injection works. Sometimes it does. It is worth a shot." "Yes, that is a good idea," the patient says, clearly relieved that the surgery is not the only option. Specialist: "But that will have to be done in the outpatient clinic." The patient seems disappointed. She had hoped to have it taken care of by this doctor at the center. "Can I call the hospital?" the patient asks. Specialist: "I will write a letter to your general practitioner, who can handle it from there."

This description of one of the ophthalmology consultations at the Primary Care Plus center is typical for the way this Primary Care Plus specialist interacts with his patients. During most of his consultations, he speaks in local dialect and he jokes about even the most serious things. Conversing in a joking manner makes most patients feel at ease in a situation that could very well make them nervous. Afterwards, most of his patients express appreciation by describing it as "pleasant", explaining that they liked the atmosphere and the doctor himself, often because him speaking in local dialect made them feel like "he is one of us".

Pleasant experiences alone, however, do not make a consultation patient-centered. Epstein and Street (2011, p. 100), for example, point out that some hospitals, in the name of patient-centeredness, adopted "models used by boutique hotels with greeters, greenery and gadgetry" (Epstein \& Street, 2011, p. 101). These will certainly improve the patients' experience, Epstein and Street argue, but they do not say much about patient-centeredness in its "essential and revolutionary meaning". At the core of the current aspiration to deliver patient-centered care, I explained in the second chapter, is a move from the dominance of "a paternalistic model of communication" towards more democratic interactions, in which the patient is asked to autonomously participate in decision-making that affect her life and her body (Taylor, 2009, p. 150). ${ }^{34}$ In the document analysis, I found that patient-centeredness in Primary Care Plus acquired yet another meaning. Here, patient-centeredness became a notion that refers to the neighborhood character of care: Care is delivered right at the doorstep of the patient, in a friendly and accessible way. In literature about patient-centeredness, much research work is done to determine its correct (re)conceptualization, to develop frameworks and realiable tools for assessing and

34 While often framed as a matter of giving patient autonomy, other, more critical voices, have shown how this shift proves to be problematic in practice. Some critics have pointed out that patients often lack the ability to make an autonomous choice because of their medical condition, or that people in general are not good atmaking decisions on the basis of numerical risk estimates or other significant percentages. Mol (2008) has brought another problem into view: A logic of choice produces a different organization of health care than a logic of good care. Organizing health care based on a logic of choice can thus be detrimental to the practices established in the past to ensure good care. 
implementing it, and to critically question the lack of conceptual clarity (Castro et al., 2016; Funnell, 2016; Håkansson et al., 2019; Hudon et al., 2011; Ishikawa et al., 2013; Langberg et al., 2019; Mead \& Bower, 2000; Taylor, 2009).

In the second chapter, I argued that although the meanings of patient-centeredness are diverse, they do have in common that in one way or the other, they are phrased in what Pickering (1995) termed the "representational idiom". This is most visible in the scientific quest to correctly conceptualize what patient-centerededness entails, how to define it and how to measure it. Such research efforts attest to a belief that it is possible to unproblematically represent a quality of something out there in the world and to pin down its essence that can be measured objectively (Hughes et al., 2008). In the context of Primary Care Plus, patient-centeredness has become a set of conditions that need to be met, a checklist that instructs organizers to deliver care according to a preset idea of what it entails. Through a relocation to the "neighborhood", fresh coffee in the waiting room, an accessible, small-scale and friendly, "village-like" atmosphere, the patient experiences are improved. By monitoring the five items of patient experience on a 1-10 scale, the delivered care can be assessed on its patient-centeredness; thereafter, the fit between patients' preferences and the organization of Primary Care Plus can be adjusted for further improvement.

Looking at the above ophthalmology consultation, however, gives a more conflicting image. The patient is satisfied afterwards, like most of the ophthalmologist's patients whom I spoke to during the ethnographic research. She felt at ease in the consultation room. Applying Langberg's (2019) dimensions, biopsychosocial, patient-as-person, sharing power and responsibility, therapeutic alliance, and coordinated care, as introduced in the second chapter, gives the following impression. The doctor, who as a person has developed his own way of connecting with his patient, even manages to build a somewhat eccentric but strong therapeutic alliance with her even though he spends a considerable amount of time behind a computer screen with his back turned towards her. The patient's trust in the doctor grows during the consultation - at the end, she is disappointed he will not be her doctor at the hospital - and he not only diagnoses the illness but also inquires how the patient as a person deals with a tearing eye. He seems to have adopted a biopsychosocial approach, helping the patient to start accepting her eye condition, for which there is possibly no effective remedy. But are the interactions in this consultation an example of inclusive and autonomous patient participation? Towards the end of the consultation, the specialist shares power and responsibility by giving the patient three options - a surgery, an injection, or to do nothing at all - but his sewer analogy has a greatimpact on the decision-making procedure as it gives the patient little freedom to choose an option other than the conservative ones. 
Thinking and writing from the representational idiom, the above interactions could be either dismissed as authoritarian or appraised as an intelligent way to involve a patient who is not eager to do so naturally. Both is possible, depending on what is emphasized in the explanation. In this chapter, I again take up the performative idiom. This allows for taking the ambiguous consultation as a source of insight about how patient-centeredness is done, instead of immediately dismissing it. With such an approach, patient-centeredness is an interaction that takes shape in the context of emerging human and material agencies in the consultation room. With a performative idiom, the consultation becomes a mangle of practice, in which patient, doctor, tearing eye, and present and absent apparatuses resist and accommodate each other. In the course of this chapter, I will analyze four examples of interactions between patient and specialists in the Primary Care Plus consultation rooms in order to answer the questionhow patient-centeredness takes shape in practice.

\section{AGENCY AND MORALITY AS PRACTICE}

Taking up a performative idiom means taking up a practice understanding of agency and morality. In the previous chapters, I have introduced the practice turn as the approach that focuses not on agency, i.e. the actions and intentions of humans, or on structures as the determinants of such actions but on the interaction of people with one another and with their material environment (Schatzki, 2001a). The practice approach was developed out of a dissatisfaction with strongly rationalized notions of agency and the social in social theories of sociology and economics (Reckwitz, 2002).

Viewing the above consultation as a practice instead of a deliberation among two rational humans with preferences and intentions has three implications for understanding agency in the context of the consultation room. First of all, as explained in the previous chapters, the notion of practice at the core of the practice approach minimally denotes a "set of activities" (Schatzki, 2001a, p. 11) that somehow cohere in order to form a practice. There are two ways in which such coherence is explained: Activities are habitual, embedded in taken-for-granted routines inscribed in bodies and materials (Cohn, 2014; Selinger \& Whyte, 2010; Swidler, 2001) or understood normatively (Rouse, 2001), as shaped by the norms that are produced and reproduced within such a practice. In this dissertation I combine the two approaches. If a rationalist approach to agency were adopted in this research, the doctor would be viewed as a professional who does or does not act according to standards of patient-centeredness. To judge the patient's interactions, I might question whether or not she is acting according to her (stable) preferences and intentions. Instead, from a practice view, the way the interactions unfold is understood as a form of 
(routinized) behavior that is shaped over time by norms that are constantly being produced and reproduced through the interactions. Secondly, the interactions under study are taken as fundamentally situated. This means that they are not first and foremost results of intentions and/or rational deliberations preceding the acts, but they are shaped by a material and social context that exists alongside it. Third, because these interactions are situated, they cannot be predicted or controlled, but, at best, the many contextual factors can be traced and reconstructed in order to understand what happened and to possibly introduce improvements.

A further set of differences that comes with the performative idiom or, more generally with the practice approach, pertains to morality. In this approach, morality is understood as a form of routinized behavior (activities as routinized habits) or/ and as continuously shaped by produced and reproduced sets of norms (normative understanding of practice), embedded in a certain practice. Morality is then not part of the inner self, nor a set of rules and procedures that leads us to unambiguous outcomes, but the diverse practical ways in which we, as "beings for whom things matter" (Sayer, 2011, p. 19), engage with the world and with people around us (Swierstra, 2015). Applied to this case, the practical ways in which the ophthalmologist and the patient jointly determine the right decision in the particular situation is a question of morality in practice, because it is shaped by embedded norms about what good health care entails, or by routines that have emerged through working with such norms. Morality, as the practical routines for determining the right decision, consequently becomes ambiguous. What constitutes the rational choice is not the outcome of an impersonal mental process, but something that emerges out of the social and material interactions unfolding in practice, a process that is shaped by the practical routines that are employed for distinguishing right from wrong.

\section{JUSTIFICATION AS PRACTICE}

In this chapter, I will not develop a checklist capturing the essence of patientcenteredness or how it should be conceptualized or measured. Instead, I will explore the practical routines and norms that emerge in the interactions between doctor and patient in order to enact good patient-centered care while achieving durable treatment decisions. For the purpose of making such routines and norms explicit and analyzing how they align, compete, and clash, I use Boltanski and Thévenot's (2006) seminal work on justification to substantiate the notion of morality as practice.

For many years, Boltanski and Thévenot have ethnographically studied how people in work settings (De Recht \& Co, 1992) achieve decisions together despite their differences. When observing these interactions, they focus on how people justify their own position and criticize those of others. This, according to them, 
is an important part of how we interact with one aother. Although we often disagree about what is the best decision in a certain situation, most of us strive to have our positions withstand a test of justification. However, such justification acts are commonly neglected in the social sciences (Boltanski \& Thévenot, 2006; Sharon, 2018).

Boltanski and Thévenot thus start from the observation that people are evaluative beings, who constantly assess options and give reasons. However, different people, as well as the same people in different situations, do this in a variety of often conflicting ways. The individual ways in which people justify and criticize are not merely subjective preferences or matters of opinion; they are each part of broader, more general sets of coherent convictions of individuals about the most important priniciple at stake (highest common good) in a particular situation. Boltanski and Thévenot refer to these sets of convictions as "coherent vocabularies of argumentation and justification that are each organized around one vision of the common good" (Sharon, 2018, p. 4). In their work, Boltanski \& Thévenot (2006) present a typology of six of such sets of ideas that they refer to as "orders of worth", 'market' (economic growth), 'industrial' (efficiency), 'fame' (reputation), 'domestic' (emotional proximity), 'inspired' (inspiration) and 'civic' (collectivity). The list was not meant to be exhaustive;in later work, the authors themselves and others suggested to extend it to include other types of orders of worth, such as the 'project' (innovation and the network) (Boltanski \& Chiapello, 2007), 'ecological' (Lafaye \& Thevenot, 1993), and 'vitality' (greater health) (Sharon, 2018) orders.

Defined as vocabularies in the previous paragraph, others have argued that such sets of convictions do not merely "contain words' or 'accounts'" (Thevenot, Moody, \& Lafaye, 2000), they also entail ways of measuring and testing values, and evaluating and prioritizing objects, actions and people. While most of the authors mentioned in these paragraphs rely on verbal sources for their analysis, Pols (2006, p. 79) in contrast, inspired by Boltanski and Thévenot's approach to analyzing ethnographic data from two a psychiatric wards, described "repertoires [orders of worth] of washing in psychiatric nursing, each containing a different ideal of citizenship". Orders of worth thus not only shape how we justify and critique but also how we conduct our daily activities with regard to — in a medical context — enacting good care.

The core question in the research of Boltanski and Thévenot (2006) is how, inspite of their often profoundly diverging convictions of what is right or wrong in a particular situation, people routinely succeed in making decisions together without using force or violence. The answer to this question lies in what Boltanski and Thévenot (2006) denote "operations of justification and critique". With such an 
operation, a person uses the order of worth deemed appropriate in the particular situation to lift the situation to a more general level. In the observations regarding the above example, by using the sewer analogy, the ophthalmologists does not merely speak about the specific intervention that will or will not be performed on the patient's tear duct but about the intervention in the context of this type of treatment in general. Using an imaginative analogy, which does not inform but illustrate how such interventions commonly play out, is part and parcel of the protective, fatherly role that corresponds to a domestic order of worth in which emotional proximity is the most important common good. Note that I use the word "fatherly", here and in the remainder of the chapter, not as an attitude or relation reserved for men or as the expression of a biological or caring relationship; it is meant in a transitive sense, referring to the traditional role of the father within a family. Despite being outdated in the family context, the symbolic, ideal type of this identity now constitutes other types of relations, attitudes and roles beyond the family, recognizable in operations of justification. An example is the Dutch term Vadertje staat, which can be translated literally as "fatherly state", a paternalistic state that restricts freedoms of its citizens to protect them against themselves. The ophthalmologist's choice of words and his approach to interacting with the patient imply such a role and that the domestic repertoire is important to him as a doctor in this consultation with this specific patient.

Thinking, speaking or acting according to one order of worth is a way of organizing a messy world. Different people will employ different orders depending on the situationthey find themselves in. As such, an order of worth is not a "perspective" or "a point of view" that is attached to a certain position, a profession, or a certain kind of expertise. The ophthalmologist in these observations used an imaginative analogy, possibly viewing his patient as if she were his child whom he needs to take care of and who depends on him for her flourishing. Another doctor might have seen her as a stakeholder of an organization that needs to run as efficiently as possible (industrial). In that case, he might have presented the patient various risk statistics, which, within the industrial order of worth, are seen as the appropriate neutral way of representing what the patient can expect from the treatment. However, it could be otherwise as well. He might consider the market order of worth to be the appropriate constellation and thus economic growth to be the highest common principle. He would then consider the consultation as an opportunity to sell treatments, possibly making use of a different set of objects, such as shiny brochures, in order to maximize profit.

Of the nine orders of worth introduced above, the civic order of worth is important as well for analyzing data in the remainder of the chapter. Within this order, the most important common good is the well-being of the social collective, the group 
of equal citizens each with rights and obligations that make up a democratic society. To illustrate, if the ophthalmologist had considered the civic order of worth appropriate for this consultation, he might have felt it to be crucial to take each and every patient as an autonomous decision maker, who has a right to receive the best care and should thus be informed as best as possible.

Being a doctor in a hospital, or a patient visiting a doctor in a hospital or another care situation, does not necessarily come with a predetermined style of decision making. In the remainder of the chapter, I take mobilizing orders of worth as not merely a matter of uttering words that represent a state of affairs but as part of a process in which the practical routines of understanding, deciding, implementing and evaluating are constituted. Employing such an order creates a world in which types of objects, words and persons acquire specific meanings and thereby a place in the hierarchical order. Orders of worth occur individually, they clash, or they coexist unproblematically in the decision-making process between doctor and patient. Importantly, we will see that patients and doctors may combine more than one order of worth. This is what Boltanski and Thévenot (2006) call a compromise: A position is justified by appealing to elements of two different orders of worth placed in a somewhat coherent frame. This is a common way of navigating differences; however, it often results in positions that are less durable. Different orders of worth come with different sets of appropriate tools; Langberg's dimensions, which move the patient center stage as an autonomous decision maker, are a more appropriate tool within a civic order of worth than in an industrial or domestic one. The notion of patient-centeredness itself could, however, be incorporated in any order of worth. Yet, in each world it would acquire a different meaning. By viewing the process of making a treatment decision in the consultation as a practice of justification, I go beyond the usual perspective of the clinical encounter as a privatized interaction between patient and care provider. The encounter is then not merely shaped by its environment but also by the personal histories of agents and by systematic changes outside the consultation room, as also outlined by May (2013) and Mol (2008).

\section{MORAL JUSTIFICATION WORK}

Combining Boltanski and Thévenot's (2006) framework with the practice approach, I see the orders of worth as part of the possibly conflicting norms embedded in 
practices. ${ }^{35}$ In order to explore how patient-centeredness takes shape in practice, I will trace the interactions of human, social and material agencies as conflicting orders of worth.

Looking again at the ophthalmology consultation described in the observations at the beginning of the chapter, what stands out is that the pleasant atmosphere of the consultation is created by the doctor speaking in local dialect and making jokes. His behavior quickly creates an intimate atmosphere and great trust by the patient in the doctor's judgement. This aligns with the analysis in the previous section, in which the domestic order of worth, which centers around emotional proximity, was already identified in the doctor's operations of justification. As noted above, the domestic order is used to also characterize non-familial relations that do show similarities to the model of the traditional family. In the present case, the doctor resembles a father; his worthiness as a person is accompanied by blind trust in his decisions.

Giving the patient a choice between different trajectories, as the doctor does towards the end of the consultation, disagrees with the domestic order of worth. As explained above, putting the autonomy of the patient center stage fits the new patient-centered model of communication, in which a doctor is supposed to make a diagnosis and subsequently give the patient treatment options to choose from on the basis of information about prognosis, risks and side-effects. As pointed out earlier in the chapter, this is seen as a profound shift from the former times, in which the doctor-patient relationship was commonly understood as a hierarchical one: The doctor decides what is best for the patient, and the patient obeys.

From Boltanski and Thévenot's framework, the new autonomous role of the patient could also follow a market logic: With the marketization of health care, the patient has become a customer for whom health care is a service (May, 2007). Within this order of worth, patients are seen as autonomous decision makers, who, in the right circumstances, can make rational decisions on the basis of a cost-benefit assessment of the different treatment options. After the shift to a market-understanding of the patient-doctor relationship, patient satisfaction has largely become a crucial factor in health sciences research, because it provides insight into the

35 It is not evident that Boltanski and Thévenot's work can be combined with the practice approach. Thévenot (2001) elaborates on the differences between his notion of a "pragmatic regime" on the one hand and the notion of "practice" on the other. He sees two great problems in practice theories:first, what he calls their "lack of realism" (p. 64), i.e. they "do not provide a good account of our dynamic confrontation with the world."; and second, the absence of a "moral element" in practice theories, "which shapes the evaluative process of governing any pragmatic engagement". For this study, I propose that by adopting Pickering's (1995) understanding of practice, in which the material agencies are one of the emerging agencies in science as a field of force - in the context of this study,the practice of managing and delivering Primary Care Plus - and by expanding the understanding of practice as a set of habitual activities by Rouse's (2001) normative understanding of practice, these two major tensions between the practice approach and the work of Thévenot are largely solved. 
preferences of the patient-as-consumer, and it thus partly determines the success or failure of health care projects. However, such emphasis on patient satisfaction contradicts the domestic order of worth that the doctor has adopted here. Within that order, an outcome measure such as patient satisfaction would be a rather meaningless and superficial impression of the quality of care. Since the doctor is a better judge of what is good for the patient than the patient herself, her judgment of the medical problem and the best treatment should always have priority over whether or not this decision will satisfy the patient.

For the eye doctor, however, giving the patient a choice is not employed as a market logic device. Even though he is aware of these contemporary expectations, as most doctors are, they obtain a different meaning and function in the consultation and in his subsequent reflections about it. Patients, he explains, often take the decision to have surgery too lightly, despite its considerable risks of complications. By first drawing the sewer analogy and then having the patient make a choice, he differentiates between patients whose medical problems dominate their lives and those who are able to lead happy lives inspite of the inconveniences. According to him, after such an extreme depiction of the procedure, patients in the latter group would say: "Oh, but the problem is not so bad after all." In agreement with the above analysis, the analogy is thus a domestic device; it lifts the particular medical intervention being considered to a more general level and gives the specialist more personal and emotional access to the personal life of the patient than merely presenting risk numbers could have. From the viewpoint of the doctor, this makes sense, because patients are considered notoriously ill-equiped to assess such risks by themselves. Within the domestic order of worth, emotionally inducing a patient's response through an imaginative analogy is a meaningful process with the most informative outcome possible.

While the doctor depicts this form of interaction as a means to uncover an already existing state (the hidden 'real' preferences of the patient), the reality of practice reveals something different. After the consultation, the patient explains: "This is just a minor inconvenience. If it could be improved by merely pricking a hole in it, I would certainly do it. But constructing a whole new sewage pipe? (...) That seems too complicated." The patient, who saught consultation to discuss a problem that was "a serious nuisance" to her, now leaves the room dealing with a "minor inconvenience". This change in her perception of the problem was prompted by the doctor's use of the sewer analogy. The "small procedure that could fix the problem" had become the "construction of a new sewage pipe".

This analysis shows that in carefully tracing the interactions between patient and doctor and their explanations of these afterwards, we can discern how interactions 
are shaped by an order of worth. It is clear that neither the patient nor the specialist has stable preferences. While the changes are small, none of the elements that are involved in the decision-making process are predetermined. Neither the patient's experience of her medical problem nor the doctor's opinion about which intervention may cure the tear duct blockage are stable ingredients. The preferences and the fit between medical problem and right intervention emerge in the interactions between doctor and patient in the consultation room.

Departing from the above analysis, I argue that articulation work (Strauss, 1988) - introduced in the previous chapter as the mundane and invisible work required to make organizational processes run - does not only have a temporal, spatial and knowledge dimension but a moral one as well. In his own work, Strauss mainly stressed the temporal dimensions of articulation work, i.e. the efforts on the work floor to make work processes run fluently in terms of temporal planning. Bardram and Bossen (2005) added an emphasis on space, i.e. the efforts required to move objects and people in space in order to accomplish organizational tasks. In the second chapter, I introduced the knowledge dimension of articulation work, i.e. the efforts to sufficiently attune knowledge differences in order to enable collaboratively achieving organizational decisions. When analyzing the above consultation, I illuminated yet another dimension of articulation work; in this case, it is not a temporal, spatial or knowledge one but rather one that pertains to morality, i.e. the calibration of differing understandings of what good care entails that are held by the participants of the consultation. Combining Boltanski and Thévenot's work with the practice approach, I have shown how such work is also embedded in the institutional, material and social context in which the consultation takes place. In order for patient and doctor to achieve a durable treatment decision together in the consultation room, efforts are required to align moral worlds, i.e. to attune the emerging, conflicting orders of worth pervading the practice of delivering Primary Care Plus sufficiently. I will denote this type of effort "moral justification work" ${ }^{36}$, which often has as much a social as a medical character. With such moral justification work, the patient and doctor move towards finding a compromise between their changing preferences, interpretations, actions, and the moral norms embedded in the care system itself. In the course of the consultation, the justification work shapes how patient and doctor understand what good care entails. In this case, the con-

36 Oldenhof et al. (2014), too, use the term "justification work". They have used it in their research for analyzing the efforts of managers in health care to find or maintain compromises when orders of worth are conflicting, thus showing much overlap with how I apply the term in this dissertation.

The term additionally resonates with Van de Werff's (2018) notion of value work, i.e. the efforts involved in the "attribution of value to something, and/or the assessment of something as valuable". Both notions revolve around the counterintuitive idea that it is not self-evident that certain things have value or for certain mundane processes to run well. Instead, continuous effort is required. 
sultation consisted of various examinations, closely listening to the patient, drawing analogies, and building trust through jokes, local dialect and knowledge. The patientcenteredness that emerged in this consultation is not merely about taking into account the preferences of the patient, her personal life, and her experiences of the daily nuisance of her tearing eye, but it is also about creating space for them to undergo change in the process.

\section{PATIENT-CENTEREDNESS AS A ROUTINE SHAPED OVER TIME}

The second consultation that I analyze in this chapter is an internal medicine consultation. The patient visited the center to discuss his overactive bowels. During the short interview before the consultation, he explains why he dislikes his general practitioner's decision to send him to the Primary Care Plus center where he does not know the specialist. Justified within a domestic order of worth, he emphasizes that the more familiar a doctor is with him and his numerous medical problems, such as his regular doctor at the hospital, the better (common principles of proximity and trust). Within the industrial order of worth of the Primary Care Plus intervention, it is justified to bypass the new center only for medical reasons, not for notions of familiarity and trust. Taking their role as gatekeepers seriously, many general practitioners are very hesitant to deviate from this norm. When entering the consultation room, the patient is rathertense and suspicious, because he feels his domestic preferences clashing with the industrial considerations due to the way he was referred.

During the consultation, specialist and patient have a lengthy conversation about his medical problems: overactive bowels, heart palpitations and IgA deficiency (acongenital disorder of the immune system).The upcoming birth of his second child and recent conflicts at his work come up as well. Following the anamnesis, the specialist does a lengthy physical examination. The interaction below takes place towards the end of the consultation and describes how specialist and patient decide what should be done next.

The specialist opens the results of the latest blood tests on her computer screen. The tests show "no signs of inflammation", a "kidney function normal" for the patient's age, and a few "minor deviating values" regarding his "liver cells". "There are only two problems," she concludes, "the IgA deficiency and the deviating liver values. I will advise your general practitioner to order an ultrasound of your liver. Besides that, I will contact your specialist in the hospital to see if there is anything else we need to do."

The patient is still somewhat dissatisfied: "I would like to get a check-up at the hospital every few months." Specialist: "Let me first talk to the specialist about it. 
And you need to make an appointment with the general practitioner to see how to proceed from here. Any other questions?" Patient: "I am still worried." Specialist: "You have coped with these problems your whole life. You have grown up with them. There are only some loose ends. Some things you need to do to prevent problems in the future." "Can those values go up again suddenly?", he asks still worried. Specialist: "Until now, it hasn't bothered you too much. So, what can you do? Just go on with your life. You can turn 100 with these kinds of problems."

Patient: "Is there anything I can do about the sounds from my bowels? I am ashamed when I sit in a quiet space or when I am at a meeting." Specialist: "There is not much you can do. Of course, you could just tell people about your problem. There are a lot of people who have such problems. (...) Whenever you are gassy, people will think, 'oh right, he has such and such problems.' " After some more questions, the patient leaves the consultation room. Clearly relieved, he sighs: "That was absolutely a very pleasant consultation".

In the above observations, it becomes clear how the specialist pushes patiently for a conservative treatment decision. The patient, however, is not easily comforted, which - in his case - is crucial for achieving a durable decision. He repeatedly asks for more tests and regular appointments with the specialist he is familiar with, which seems prompted by his strong need for trust. His attempt to gain trust (domestic common good) through numbers (type of reliable knowledge in the industrial constellation) is an unlikely combination of means and end. It brings to mind Theodore Porter's (1995) argument that the increasing drive to quantify policy issues, i.e. the increasing dependence of modern governments on statistics, does not stem from the increasing status of science but from the diminishing levels of trust that the population has in governing elites. In a similar vein, the absence of a trusted doctor makes the patient insecure about the care he is receiving. Repeatedly asking for more tests, i.e. more numerical measures, is unlikely an indication that the industrial order of worth is appropriate for him but rather that he doesnot feel at the right place, that he cannot trust the new doctor, who does not know him yet. This typical combination of numbers (industrial) and lack of trust (domestic) demonstrates that compromises between orders of worth can be made, in this case resulting in an industrial-domestic order of worth. Here, the characteristic domestic good of trust is attained through numbers as a tool to gain a sense of control and predictability - in this case, regarding his medical situation and the course of his illnesses. At the end of the consultation, however, the patient goes home content even though he did not get a clear diagnosis or treatment for his bowel condition, or any other tests or regular appointments. 
The specialist is one of few doctors responsible for the internal medicine appointments at the center. As a rule, she does not refer patients to a specialist in the hospital; instead, she writes an extensive advice to the patient's general practitioner and tells the patient to make an appointment at the general practice to discuss possible further steps. When she thinks that a patient needs non-urgent specialist care, she does not issue the referral herself but advises the general practitioner to refer the patient.

In the interview after the consultation, the reflections of the specialist for internal medicine are mostly structured around the aim of increasing trust and are thus mainly set in the domestic order of worth. Almost every action that the specialist seems to take naturally turns out to be part of a carefully constructed routine she has developed for her Primary Care Plus consultations as a solution to an important problem in health care: patients' trust issues. The duration of the consultation is an example. Instead of efficiently scheduling one patient after the other, the specialist has arranged a compromise with the organizational context of her consultation, which is shaped by the industrial order worth. When scheduling her consultations, planners only assign a thirty-minute consultation per hour. This allows her to take more time with a patient, often needing the full hour before the next patient arrives.

During the lengthy anamnesis of the described consultation, she sits at her desk, facing the patient, who sits on the other side. Before she starts taking notes, she turns the computer screen, so the patient can read what she is typing. The specialist explains how this helps the patient toconvey their situation:

When I turn the screen, they say, 'yes, yes, that is exactly what I mean.' It makes people feel understood (...). It also gives the patients some structure, so they will stay a bit concrete and to-the-point.

During the anamnesis, she carefully switches from personal to medical issues, giving the patient ample room to elaborate on any topic and to take his time when responding. Some of the doctor's responses are technical, but just as often, her responses have a common-sense character, which helps the patient to understand in a practical, down-to-earth way. During the physical examination, the doctor asks the patient about his medical problems, but she also compliments him on what she observes: "such beautiful slim ankles!", "what a flawless and smooth inside of the bowel!" Similarly, informing the patient that she will ask the specialist for advice, as she does in the above described consultation, is not only for content-related reasons; it is also part of her mission to restore the patient's trust in his general practitioner: 
He will have to deal with his general practitioner for years and years. He needs to trust her to be a serious doctor who has taken him and his problems seriously. Telling him that I need to consult the professor specialized in this subfield of internal medicine is a way of saying, 'this is a complicated clinical picture, I can imagine the general practitioner does not know all the ins and outs of it. (....) I also need the input of the super-specialist.'

Because of this domestic mission of restoring patients' trust, she sends all patients back to their general practitioner, even if her advice is to refer the patient to the hospital for a test or more specialized care. To other, more industrially minded specialists in Primary Care Plus, such a stopover makes the patient's trajectory needlessly inefficient. The specialist in internal medicine, however, prioritizes the symbolic function of merely advising the general practitioner, who needs to stay in charge, rather than making referral decisions herself. Within the domestic order of worth, this is reasonable because trust is not only crucial for her relationship with her patient but also for the relationship that the patient has with his general practitioner. Many of the patients she sees have multiple vague symptoms and they have repeatedly consulted their general practitioner without success, thus still dealing with unresolved issues. The patient in this consultation is exemplary for this group when he says:
Sometimes I feel that the general practitioner's knowledge and skills are too limited to deal with my problems. (...) While a general practitioner would say, 'yes, that could be the case,' a specialist says, 'if you get this and this treatment your problems will go away.'

What the above observations reveal is a routine that the specialist has developed to maximize the gain of trust in the limited context of the Primary Care Plus consultations. In terms of proximity, the Primary Care Plus centers, despite their "village-like atmosphere", seem hardly equipped for this goal. Most of the patients whom medical specialists see at the center are new to them. They may have one or two consultations with them, which do not suffice to build a solid bond of trust. Yet, the doctor believes that one reason to collaborate in the intervention is that it can help prevent endless hospital trajectories of patients, including medically redundant tests and treatments.. This motivation is similar to one of the promises of Primary Care Plus identified in the second chapter: that the relocation will improve the cost efficiency of the center. While this promise is strongly prompted by the industrial order of worth, within the medical specialist's order of worth (domestic), it obtains 
a different meaning. The problem of such an endless trajectory is not its inefficiency or the unnecessary costs but, again, its detrimental effect on the patient's trust. Justifying her Primary Care Plus routine, the medical specialist explains that $40 \%$ of the patients she sees at the outpatient clinic of the hospital do not have a medical problem; they have a "trust problem":

If they come to the hospital, they first see an intern, then the supervisor. In the meantime, several tests have been done that are not necessarily related to the problem the patient came in with. (...) If you are insecure as a patient, you will think 'oh, I must have something really serious' and become really worried. This is how they enter a cycle in which more worries cause them to demand more tests, which will cause yet again more worries, and so on.

Note that in this quote, the connection that the medical specialist makes between tests and trust is the opposite of the patient's operation in which numbers are expected to reinforce trust. While the patient aims to gain trust through numbers, the medical specialist points out that the opposite is often the case: Testing decreases the trust of patients in their own body and their own understanding of how well it is functioning.

From the medical specialist's viewpoint, the consultation described above can be marked as a successful one, i.e. a consultation in which her routine has the intended effect. In the course of the consultation, the specialist and patient build a trusting relationship, in which the patient can be more vulnerable and open, and his increasing trust in the specialist enables her to help him overcome his lack of physical confidence. The patient's initial discomfort that was caused by the clashing of the industrial order of worth of the system and the domestic one that is appropriate to him, slowly resolves when him and his worries become part of the developed routine that is likewise shaped by the domestic order of worth. Toward the end of the consultation, patient and medical specialist achieve a treatment decision that the medical specialist endorses and that the patient feels sufficiently comfortable with.

I draw three conclusions from the above analysis. The first conclusion pertains to patient-centeredness. The intervention was established with a low-tech neighborhood character to, among other reasons, create the right atmosphere to make patients feel at ease. As explained, this is the meaning that patient-centeredness has acquired in marketing material, reporting, and other writings and communication about the promises of Primary Care Plus. The above consultation obtains depth through the process by which the specialist carves out her routine. Thinking and experimenting from a domestic order of worth in which the most important patient 
need is trust - in doctors, their own body, and in the medical system - the specialist has developed an unexpected and detailed routine to deal with patients such as this one. Patient-centeredness in this example is not a quality of an interaction, nor the process of dealing with emerging and fading preferences of a patient, but it is a routine that is shaped over time within the strong domestic order of worth, in tension with the organization's industrial one. Here, patient-centeredness is not a matter of working according to a protocol and following a set of dimensions, but it is achieved through carefully developing and following a routine in which the doctor is constantly anticipating and working with the emerging needs of her patient.

For the second conclusion, I further elaborate on the notion of justification work introduced at the end of the previous section. In the analysis of the ophthalmology consultation, justification work was a dynamic process of making orders of worth align; in the consultation analyzed in this section, it is an emerging routine. Justification work in this consultation is a process of calibrating a care routine in the context of the emerging possibilities of the new Primary Care Plus environment and the constant interaction with patients. In this process, the specialist navigates the emerging agency of her patient; she is involved in a "play of resistance", for example when dealing with the patient's unmet needs of more tests and appointments, "and accommodation", when extensively addressing the social and medical worries of the patient in other ways. This justification work does not start and end in one consultation, it extends beyond it; after repeatedly seeing patients with similar problems, it results in a routine that is tailored to accommodate the trust issues of the patients.

Thirdly, the consultation shows how Pickering's (1995) notion of emerging human agency can be an entry point for understanding patient-centeredness. In his book, Pickering sets out to develop a symmetric approach to human and material agencies. As I explained in the first chapter, for him, a practice is "an evolving field of human and material agencies reciprocally engaged in a play of resistance and accommodation" (Pickering, 1995, p. 23). One problem in approaching the two types of agency symmetrically is that it seems self-evident that human agency has an intentional structure, whereas this is absent in material agency. Humans (often) act for reasons, whereas such motivations are absent for material things. Pickering's conceptual solution is to start from an "exact parallel between the intentional structure of the two" where both intentional structures are "wild and undomesticated" (1995, p. 18). As such, he starts from the conceptual point that both material and human agency have an intentional structure, but these seem non-existent, because there is no logical order, i.e. no rational coherence, to it. The mere difference between material and human agency is thus a practical matter, not a prin- 
cipled one. Whereas materials will never be able to bring order to the intentional structure of their actions, humans, in contrast, enroll in processes of reasoning in the course of their live, thus developing reasoning skills. As such, the intentional structures of their actions usually become more and more coherent, or "domesticated" in Pickering's terms. Studying practices of science, in this case a Primary Care Plus consultation, as playing fields foregrounds how the intentional structure of agency becomes increasingly tamed.

The process in which the specialist's routine emerges is, from Pickering's perspective, a process of domestication. This is to say that the specific personal approach of the specialist of internal medicine to Primary Care Plus care and to patient-centered care was not set before she started offering such consultations; instead it is a result of doing them, reflecting on them, placing them in a broader framework of societal considerations and personal experiences, and thus of domesticating its intentional structure. Such a process is thus emerging and temporal and can be studied from the surface. As described by Pickering (1995, p. 20):
the intentional character of human agency has a further aspect of temporal emer- gence, being reconfigured itself in the real time of practice, as well as a further aspect of intertwining with material agency, being reciprocally redefined with the contours of material agency in tuning.

Note that with such a substantiation of the intentional structure of human agency, the specialist's reflections during our interviews are not a representation of her actions and intentions. Rather, these reflections are part of the real-time reconfiguration of the intentional structure, i.e. they are part of the justification work itself in which the routines are calibrated.

\section{CRAFTING PATIENT SATISFACTION}

In the previous section, I carved out human agency as routine, built over time in a process of continuously navigating resistance and accommodation in a playing field and explicitly reflecting on it. In this section, I will scrutinize a rheumatology consultation in which the emerging human agencies are not a carefully built up routine but show similarity to a play, consisting of social interactions.

The patient at the consultation is a young woman who came to the Primary Care Plus center to consult a rheumatologist about her painful joints. She is worried that she has rheumatoid arthritis (RA) like her grandmother. In the short conversation preceding the consultation, she conveyed that she does not take no for an answer during a doctor's appointment. When she recently pushed for the removal of a mole 
that was diagnosed as benign but turned out malignant afterwards, it was not the first time she questioned a medical advice and turned out to be right.

\section{Ruling out Rheumatoid Arthritis (RA)}

At the beginning of the consultation, specialist and patient sit around the desk. "Your question is whether this is rheumatoid arthritis", the specialist reads out loud from the referral letter. During the anamnesis she asks standard questions such as "how long has it been bothering you?" and "where does it hurt?" When she asks: "have you seen any swellings?," the patient answers: "yes, the day before I went to the general practitioner."Specialist: "Do you wake up at night from the pain?" Patient: "No, when I sleep, I sleep." After a few more questions the patient describes how she experiences the pain: "It really bothers me. It feels like my fine motor skills are not working anymore. I cannot peel a kiwi, hold a grocery bag or depilate." Specialist: "Does it hurt when you do those things?" Patient: "Yes, I get stabs of pain."

After the physical examination is finished, both sit down. The specialist carefully summarizes her findings: "You told me you are in pain when you make certain movements. You do not wake up from the pain at night. You are a bit stiff in the morning and you had a swelling once. When I examine you now, I do not see the signs of arthritis that I need for diagnosing you with RA. RA comes with swellings in the joints, waking up at night because of the pain, and serious stiffness in the mornings. That is not what I hear from you. It is possible that your joint pain is caused by the hypermobility syndrome mentioned in your file, which can be treated by an occupational therapist. I cannot definitively rule out RA, but I cannot diagnose it right now either. If you get more swellings, you need to come back immediately."

In the short interview following the consultation, the patient explains why she is content about the care she received: "It made a big difference that she [the specialist] did not say, 'it is your hypermobility. That's it.' (...) but that she also explained what symptoms would lead to a RA diagnosis." With this remark, the patient refers to the last few minutes of the consultation, in which the specialist accounted for the fact that she could not diagnose the patient with RA by explicating the steps of the protocol she followed. Using a protocol is a routinized behavior for a doctor; it offers a quick and efficient way to make a decision on the basis of answers to a prescribed set of questions. As such, it functions in the industrial order of worth as a way of lifting a particular situation to a more general one.

However, when reflecting on the consultation afterwards, the rheumatologist explains that her motivation is not merely to achieve efficiency in the consultation. In this particular consultation, she chose to use the protocol because she felt the 
patient to be a specific type of patient, "the fibromyalgia patient". Fibromyalgia is a contested diagnosis. People suffering from it are known among specialists to be difficult to treat. They are demanding, because they suffer from pain daily, but it is impossible to localize the cause and there are no "hard" effective medical treatments, only treatments that help patients cope with their pain in their daily lives. The specialist explains:

She [the patient] does not have the same kind of pain [as patients with fibromyalgia], but she has the character that such patients have. She is a perfectionist; she does not stop vacuuming when she is in pain. (...) And she is very much fixated on her physical problems. (...) I read in her file that she had been shopping quite a bit for her back problems a few years ago. She went to see numerous different specialists who each gave her a different advice. (...) I told her to stop seeing specialists. (...) It could have become a big problem. That is why I tried to steer her in the right direction then, but also this time.

Fibromyalgia is a medical disorder, but in the way the specialist uses the comparison to lift the particular situation to a more general level, it becomes more of a personality trait. By building on her personal experiences with the patient and on her file, she familiarizes herself with the patient as a person in order to accommodate this when advising her:

When I look at this patient, I see that she could just move her hands like that, and touch all her fingers without any problems. In her story, she speaks of experiencing a lot of pain. Well, in that case I take the [reported] pain with a pinch of salt.

Her justification shows that behind the use of the proctocol - a tool appropriate in an industrial order of worth - lies a complex set of considerations about the patient's personal traits and personality, which are of concern in a domestic order of worth; therein, personal, intimate details of somebody's personality can legitimately inform decisions. In this consultation as well, the specialist tries to prevent an endless patient trajectory that would amount to an expensive and inefficient use of the healthcare system, but also because she believes it would have a detrimental effect on the patient's personal life. The specialist uses the industrial order of worth for the sake of the domestic consideration of caring for the patient, of protecting her against herself, i.e. against making her life more difficult by excessively fixating on her pain.

For the patient, the same device acquires an entirely different meaning. To elaborate 
on why she is content after leaving the consultation room, she refers to other recent care experiences and concludes:

As a patient, you are supposed to cooperate as a meek lamb. (...) As soon as you dare to say anything, when you stand up for your rights, you are immediately labeled as brazen, thus, as a difficult patient. I really dislike being stigmatized like that.

The patient lifts the particular examples to a higher level by referring to her rights as a patient. This operation fits the civic order of worth. As such, any particular health care situation becomes one in which all patients need to be treated equally. Basing decisions on earlier experiences of the patient then becomes an act of basing them on superficial impressions. This is not a form of good care but an unjust practice, a stigmatization. The patient, however, does not know that the specialist has taken her personal impressions of the patient into account. The patient takes the explication of the protocol at face value. For the specialist,the protocol is an evidence-based device that helps her to distinguish for which patients a treatment is sensible and for which patients a treatment would be futile and possibly harmful; for the patient on the other hand,the protocol seems to carry a different meaning. For her, it is a civic-order-of-worth device, an accepted code in an organization or group, that is, a procedure to ensure that people are dealt with equally, irrespective of their personal characteristics or background.

It is clear from the observations that in the consultation, a much broader range of concerns shape the interactions between doctor and patient than merely the concern of diagnosing an illness. The observations reveal that what is considered the right decision by the doctor as well as by the patient is not decided in advance but is determined in the interactions, i.e. through the justification work conducted by patient and specialist in the decision-making process. The patient was demanding as she arrived, leaves the room with little more information than she already had, but is nevertheless content. The specialist navigates her way through the process by selecting indicators here and there, making small informed and reasoned decisions along the way. A wide variety of elements are of importance for the process to unfold as it does: the routinized behavior of the specialist that is prompted by her impression of the patient's personality, the patient's conviction of what is fair in the consultation room, and the (way the specialist presents her use of a) protocol. These elements should not be regarded as stable ingredients that precede the decision in a linear, causal fashion, but as existing alongside it. During the process, they take shape and acquire different meanings for different people, and they have a constitutive effect on the outcome. 
From this consultation, we can draw three conclusions. First of all, a crucial part of the justification work required to make effective treatment decisions consists of a process of human reasoning (agency) aimed at achieving a treatment decision while taking a broad set of social, medical and material factors into account. This process does not follow a certain logical sequence that can be delineated beforehand but is unpredictable and emerging. It can be best described as a process of calibration, in which participants constantly take new information and new meanings into account, interpreting and reinterpreting those from within a changing order of worth or from a compromise of orders of worth. Note that in the case of this consultation, the decision is achieved not by aligning the orders of worth deemed appropriate by patient and specialist, but by merely connecting them through the protocol functioning as a link. In the context of this consultation, the protocol is like a boundary object, because it "inhabits (...) intersecting" (Star \& Griesemer, 1989, p. 393) moral orders of worth while satisfying the moral requirements of each of these worlds. Plastic enough to adapt to the needs of patients, specialist and health care, it is also a robust object, as it maintains its identity as a system for ruling out RA while going from one order to the other. The patient and the specialist are both content about how the process unfolded and about the decision that resulted from it; yet, because the protocol acquires a different meaning in the orders of worth of the patient and specialist, the processes they refer to are two different ones.

Secondly, the process emerging in the consultation is aimed at achieving a durable treatment decision, but the justification work is also concerned with the past and the future. In their emerging process of reasoning, both medical specialist and patient stumble upon dots in need of connection that they compile into a more or less coherent image. Making sense of the operations of justification that unfold in the consultation room involves an element of storywriting, a somewhat coherent bridge that is ereceted between the experiences and events of the past and the expectations of the future.

Thirdly, what is considered patient-centered in this process is ambiguous, it is not the outcome of following a protocol based on five or six dimensions nor a state that can be induced through a village-like atmosphere. It is a notion with plural meanings, embedded in the personal, material and social context of both decision makers. For the specialist, patient-centeredness emerges from lifting the particular elements to a higher level within domestic-industrial order of worth, a process in which she places the reported symptoms in a broader set of clues gathered during and after the consultation. For the patient, patient-centeredness, placed in the civic order of worth, acquires a contradictory meaning: it is about treating patients with the same symptoms equally. The two different logics are compatible, resulting in a successful 
consultation, with a satisfied patient and a specialist who feels she did what was right for the patient. However, as the outcome of a process with contingent elements , it could have been otherwise as well.

\section{NAVIGATING THE MATERIAL AGENCY OF ABSENT HIGH-TECH DIAGNOSTICS}

In three consultations analyzed above, the playing field that I discussed was mainly populated by human agencies, with an occasional protocol or analogy. The fourth consultation, in contrast, centers around an important material agency present in the Primary Care Plus consultations. As explained, Primary Care Plus is a facility for delivering specialist care by offering merely expertise and experience of medical specialists, without the high-tech diagnostic options of the hospital. The absence of such diagnostic procedures occasionally emerged as a material agency in the consultation room, in need of being navigated.

The next observations stem from another ophthalmology consultation. This time, the patient consults the eye doctor because her optician could not get her prescription glasses right. The consultation starts with a short conversation between the eye doctor and his patient, followed by a vision test and a slit lamp examination. The following observation is from the end of the consultation, when the ophthalmologists explains to the patient what he has found and what he advises to do about it:

\footnotetext{
“There are two issues. You have a bit of cataract. I don't think you need to do anything about that right now. Also, your retina is getting thinner and there are little spots on it." He continues to ask some more standard questions, and then: "Do you have tiles in your bathroom?" The patient seems surprised while answering the question affirmatively. Eye-doctor: "Check now and then if the lines are still going straight when you look at them. When they start meandering, then you should immediately come to the hospital. That means the small blood vessels in your eyes are starting to grow rampantly. But for now, we should not jump to conclusions. (...) Just come back if your sight is getting worse."
}

As explained in chapter one and two, the low-tech environment of Primary Care Plus is an important element of its efficiency-for-sustainability rationale. Promoting efficiency as a means to protect health care as a common good from medically redundant use, the absence of high-tech apparatuses is mostly justified within a civic-industrial order of worth. In the promise of patient-centeredness, the low-tech character sometimes emerged in a domestic order of worth as part of the friendly and accessible atmosphere of the centers, in contrast to the large, white, high-tech bureaucratic building of the hospital, in which patients hardly feel at ease. 
The absence of diagnostics for the eye consultations means that the specialist only has direct access to two apparatuses: a slit lamp examination and a vision test, both of which he uses in almost every consultation. For a medical specialization that mainly observes through technical devices, this is quite limited. During the slit lamp examination in the consultation, the specialist detects an early-phase macular degeneration, which in later phases can lead to blindness. At the outpatient clinic in the hospital, there is a new eye clinic with roughly twenty different apparatuses that are being used daily. There, doing an OCT to determine the baseline value is a standard procedure in case of any phase of macular degeneration. As such, the procedure is part of an efficient work flow systematically designed to guide patients through the procedures in a pleasant and efficient way. Such an industrial-order-of-worth design is made to accommodate the needs of patients who are assumed to feel unsatisfied after spending a day in hospital waiting rooms because of a a few tests. However, the patient from the above consultation, who is now diagnosed with an early phase of macular degeneration, is sent to the Primary Care Plus center and is thus notadmitted to the highly efficient work flow of the eye clinic. After finding the spots on her retina, however, the specialist could refer her there for the OCT anyways. Instead, he decides to refrain from such a referral and keeps the treatment low-tech.

In the understanding of Primary Care Plus' patient-centered "care at the doorstep", the conservative treatment decision to have the patient regularly look at the joints between the bathroom tiles seems effective since the patient feels reassured. During the short interview after the consultation, the specialist emphasizes that he could only make such a decision because he knows it is medically acceptable. However, in a later interview, he mentions a similar situation to explain that making a decision is more complex than discerning what is medically acceptable from what is not:

Clearly distressed by the situation the specialist exclaims: "If we look at this patient, in the hospital it is a standard procedure to make an OCT. (...) Such an OCT is a small procedure for the patient. It is not burdensome at all! It takes only a minute to get the needed information!" In the Primary Care Plus center, it is different: "Imagine you have a patient of around 80 years old. He has a bit of vision decline, though still seeing $70 \%$. On the retina, some shifts in pigment are visible. (...) When I see the same patient in the center, I think, 'well, he is still seeing $70 \%$, he is managing. Should I send this patient who is a bit older, who might have come with special transportation, who might be dependent on his rollator to the hospital when he has already made such an effort to come here?” 
In the eye doctor's operation of critique, he lifts the particular situation to a more general level by introducing an example that brings the difficulties of such a type of treatment decision into view. The older, dependent patient entering the imagined situation evokes feelings of care in the listener and as such creates emotional proximity. With the operation, the ophthalmologist relocates the decision from a civic-industrial order of worth to a domestic one, in which other kinds of concerns become important. What is considered the wisest thing to do is not stable over time or from context to context. The decision to order an OCT acquires a different meaning when making decisions regarding an elderly patient in the Primary Care Plus center than in the industrially organized outpatient clinic. A routine action that is part of an optimized process in the high-tech eye clinic and does not require any time or effort of the patient or the specialist, becomes a burdensome procedure, a complicated journey, which takes time and might hardly be feasible depending on the condition of the patient. Shifting from context to context inevitably entails a shift from the one order of worth to the other, modifying what counts as a valid argument or concern and what not.

This is an example of material agency. The meaning of the procedure, the appropriate order of worth, and how the specialist prioritizes values are shaped by the social and material context, in this case the absence of otherwise routinely used high-tech apparatuses. How much should a specialist care for his older patients, who are less mobile, or for others, who can hardly find time for a hospital visit? In the hospital, this is a simple decision for the specialist. However, because of the neighborhood setting, the same decision becomes a difficult one, which involves a number of concerns that the specialist in the hospital does not need to face.

When navigating the material agency of delivering Primary Care Plus as a mangle of practice, it is not easy for the ophthalmologist to make patient-centered decisions. When the doctor reflects on his decision as a deviation from his habitual way of acting in the outpatient clinic, he sighs, he swears, and he puts on a worried face, as he recalls other annoyances:

Why is it the specialist who has to make an effort to come to the location? Sometimes I arrive gasping for breath, because something in the hospital has taken more time than expected or I had to do an emergency ultrasound. (...) The time it takes to travel here, I could have used to see patients.

While the new situation of the Primary Care Plus center promised patient-centeredness, shifting the location of specialist care creates new challenges to its patient-centeredness. Having to deal with these challenges causes what we could 
call moral discomfort. ${ }^{37}$ The specialist follows the values he deems appropriate in the situation, but this is precisely what makes him question the setup. Why should he take values into account that have become irrelevant due to the way the hospital consultations are organized? More contextual factors are relevant than the designers of Primary Care Plus as neighborhood care could have foreseen. Such problems become apparent when considering patient-centeredness as more than a tick-box exercise and instead as an emerging care practice.

From the analysis of this ophthalmology consultation, I can draw conclusions concerning the justification work conducted during the consultation and the patient-centeredness that emerges. First of all, as also became clear in the previous section, achieving treatment decisions requires justification work, which consists of navigating unexpected agencies emerging in the practice of Primary Care Plus. Most important in the context of this ophthalmology consultation is how the eye doctor navigates the resistance emerging out of the material agency of absent diagnostic possibilities. Going from high-tech eye clinic to low-tech neighborhood center comes with shifts in appropriate orders of worth, which necessitates even more complex and possibly frustrating justification work. Again, in this case as well, the patient-centeredness emerges out of these calibration processes and is not a stable set of conditions. As the hypothetical example showed, it is shaped by many variable material and social conditions and how the doctor navigates these. Instead of improved patient-satisfaction, the relocation could cause patient-centeredness to have detrimental effects. By taking the patient and his needs into account, the justification work becomes more extensive and demanding than in the routine-like situation of the high-tech eye clinic.

\section{CONCLUSION}

In this chapter, I departed from one of the main promises identified in the second chapter, namely that relocating specialist care to a neighborhood location with a village-like atmosphere would improve its patient-friendliness. By adopting a practice approach for exploring the ethnographic observations of the interactions between patient and specialist in the consultation room as well as their preceding and subsequent reflections about it, I traced how patient-centeredness took shape in that specific context. From the explorations, I draw two overarching conclusions. The first pertains to patient-centeredness and the second to justification work.

In the broadest terms, patient-centeredness is not a quality that can be measured by using a checklist, or that can be acquired by improving the satisfaction

37 I use moral discomfort here as a weak version of "moral distress", which means "knowing what to do in an ethical situation, but not being allowed to do that" (Savel \& Munro, 2015, p. 276). In a similar vein, the specialist knows which decision would be optimal, but the situation is making him take different considerations into account. 
level of patients or by following a protocol. In the course of the chapter, patientcenteredness appeared as an emerging characteristic of the interactions, a routine, a plural notion embedded in the moral, social and material contexts in which patient and specialists act, and an ideal that complexifies the transition of providing specialist care from one setting to another. In the first rheumatology consultation, the treatment preference of the patient was not a predetermined constant; it was established not through providing mere information about the procedures and its risks but through exploring the treatment options using an imaginative analogy. This allowed the patient to assess feelings that risk numbers could never evoke. In the consultation for internal medicine, I encountered patient-centeredness as a routinethat was carefully developed through continuously engaging with and caring for patients who lack trust in themselves, the doctor and their bodies. In the third rheumatology consultation, I showed how notions of patient-centeredness can differ according to which orders of worth are deemed appropriate, but that these differences can nevertheless be sufficiently attuned with a moral boundary object. And finally, the second ophthalmology consultation illustrated how the ideal of patient-centeredness complexifies the transition from context to context. Placing the needs of a patient center stage in the high-tech eye clinic occurs naturally, while at the Primary Care Plus center, it involves a complex operation of viewing and estimating the importance of different values for patients.

Carving out patient-centeredness in these four consultations is an example of exnovation. When analyzing these consultations, I did not compare observed actions and results to a standard nor did I study whether preset conditions where present, and I did not collect satisfaction outcome measures with a questionnaire afterwards; instead, I concentrated on tracing how patient-centeredness is implemented in the consultation room. In the context of this study, this means I explicated the interactions between specialists and patients that enabled them to make a durable treatment decision. Most of the specialists I observed during the ethnographic observation are quite aware that patients need to feel part of the decision-making process in order to achieve their acceptance. In the analyses of this chapter, I have outlined the ways in which these specialists interact with patients that extend beyond straightforward exchanges of information. The specialists whose consultations I attended and with whom I subsequently reflected on their routines of enacting good care have developed their own personal ways of doing this. Explicating these interactions is thus a process of exnovation: making visible how it was done, there and then. This enables reflection on the more and less desirable parts of how they implement patient-centeredness, and it enables doctors to learn from themselves and from one another. As mentioned in the introduction of the 
dissertation, it is precisely because professionals have often become blind to many possibly beneficial routines and habits they have developed in their daily work, even when it is new, that such reflections are an important and rich source of insight for further developing the concept of Primary Care Plus as well as for the specialists' personal development. Notably, such a concept of learning is only possible when taking up a practice view of knowledge. From the mentalist view of knowledge, mentioned in the third chapter, in which knowledge is considered to be something residing in the mind that can be obtained through learning processes, it is illogical to want to learn from oneself; it would mean to learn something thatalready resides in the mind.

The second conclusion pertains to the notion of articulation work introduced in the third chapter. There, I argued that the work required to make organizational processes run fluently, i.e. articulation work, is not limited to ensuring that appointments do not overlap (temporal) or that the right people and objects are in the right spaces (spatial); it also entails the efforts of attuning knowledge differences sufficiently. In this chapter, I explored the moral dimension of articulation work, substantiated by Boltanski and Thévenot's orders of worth, which I referred to as justification work. To make health care trajectories proceed smoothly, it is important for treatment decisions to be durable. Like temporal, spatial and knowledge articulation work, moral justification work entails the efforts required to make processes run fluently, in this case the efforts of attuning the diverse understandings of what good care entails, and thus what the right decisions and process to achieve it are for the specific problem and context of the patient. I have explicated and analyzed the practical operations of such articulation work by using Boltanski and Thévenot's orders of worth.

The justification work that I encountered during the ethnographic observations at the Primary Care Plus centers was diverse. In the first ophthalmology consultation, the justification work was a momentary interplay of clashing orders of worth, in which the patient's treatment preference was calibrated. In the consultation of internal medicine, in contrast, it extended before and beyond the consultation room as a careful and slow process of developing a routine for dealing with and anticipating the emerging needs of patients. This process of justification work can be understood in Pickering's terms as a real-time reconfiguration and domestication of the intentional structure of human agency. The third consultation, rheumatology, showed a combination of the above findings. The justification work by which patient and doctor achieved a durable decision consisted of both routine and calibration justification work. What was different this time was the clash of orders of worth, which patient and specialist were able to avert. Despite deeming a dif- 
ferent order of worth appropriate and thus holding different understandings of what good care entails, they managed to sufficiently attune their experience of the process unfolding in practice. The protocol explicated by the specialist played an important role in this process. It proved to be a malleable device that acquired a different meaning in different orders of worth and, as such, as capable of attuning the patient and specialistregarding the outcome of the process, i.e. the decision itself. In this consultation as well, the justification work again extended beyond the consultation room. Justification work is the calibration work of attuning or connecting orders of worth, and the process of developing a routine, but it is also a process in which patients and actors create a more or less coherent story, in which their past and future care experiences are carefully embedded. In the second ophthalmology consultation, lastly, the justification work conducted mainly focused on navigating the emerging material agency of absent diagnostic tests. In this case, there was no tension between the orders of worth that patient and specialist deemed appropriate, but between the orders of worth that one and the same person deemed important in different contexts. When shifting to another context, different values became important, making the justification work required to achieve a durable decision- as said - extend beyond the consultation room, i.e. the specialist attempted to create coherence in the ways of working with similar patients in different contexts.

By further expanding the notion of articulation work in this chapter, I have shown how the focus on knowledge and justification work brings important processes into view that help understand what patients and specialists do to achieve durable treatment decisions. In the next chapter, I will shift my focus from the promise of patient-centeredness to the promise of cost-efficiency through relocation. I will show how tracing the moral dimension of articulation work can be an effective first step for ethically assessing an innovation, taking its rich practice into account. Reflecting on four examples of nudging in the practice of Primary Care Plus, I will answer the question how such a change in choice architecture can be done legitimately. 


\section{5 \\ TURNING OF MINDLESSNESS HOW TO INTERVENE IN A CHOICE ARCHITECTURE LEGITIMATELY}

\section{Wait and see}

The patient, who has an appointment at the rheumatology consultation hours at the Primary Care Plus center, is a woman in her mid 20s. She has been suffering from painful joints for years. The general practitioner had ordered some tests and $\mathrm{x}$-rays in 2011 and 2013. They did not show anything out of the ordinary. After, the general practitioner had not done anything to find out what is causing the pain, making her feel not taken seriously and push for a referral to a specialist. She is a bit worried now, especially after her mother has told her about a colleague, only 26 , who recently heard from the doctor that she has a serious medical condition and will probably not live for much longer than six months.

The consultation starts with the usual steps: an anamnesis, in which the rheumatologist asks questions about the pain the patient feels right now, what she has felt in the past years and whether she has any family members suffering from rheumatoid arthritis (RA). During the physical examination that follows, the rheumatologist tests the patient's joints by making movements with her arms, legs and upper body and by pushing on certain spots. Then, they assess the images and test results from 2011 and 2013 together.

Near the end of the consultation, the rheumatologist says: "The most important thing is that there is no sign of RA. Your elbow...”, she hesitates, “... I don't know what to make of that, but I think that is more likely a problem of the muscles than of the joints. It is probably caused by a bit of hypermobility syndrome." Meanwhile, touching the painful spot on the arm, she says, "But in that case, I don't understand why it hurts here." Looking at the patient's posture she says: "If you learn to use your body differently, your joints have to carry less weight." Then she switches to the painful back: "Work on your posture. Stand in front of a mirror and check whether you are standing up straight."

In the final minutes of the consultation, the rheumatologist suggests optional next steps without making these seem urgent: an MRI to see the state of the patient's tendons, an ultrasound scan to examine the elbow. "But if you want that," 
she says, "you would have to go to the outpatient clinic. We can also start with some anti-inflammatory pills and wait and see how that goes." Patient: "Soon, we will have a break from volleyball training over the summer. Maybe it is a good idea to see how my elbow feels after?” Rheumatologist: “That is a good idea. I will mention in my advice letter to your general practitioner to check after the summer if the elbow is still hurting. If it is, he can always prescribe anti-inflammatory medicines."

The interactions above can be interpreted as a typical and successful Primary Care Plus nudge. As explained in the second chapter, nudging is presented as a new policy instrument that entails directing behavior by changing the environment, in which nudgees, the people being nudged, take a decision (Thaler \& Sunstein, 2008). Nudging is based on the dual process theory (Kahneman, 2011), which departs from the idea that people act either deliberatively by carefully identifying the rational option, or automatically as a response to environmental cues. In our daily lives, we use heuristics, or rules of thumb, to enable effective and quick decision making in situations in which time and ability to process information are limited (Haselton et al., 2015). Some of these heuristics lead to cognitive biases, patterns of behavior that systematically deviate from what would be rational in the situation at hand (Tversky \& Kahneman, 1974). As explained in the second chapter, navigating their lives quickly and efficiently, the average grocery shopper would bend down to get an item from the lowest shelf when it is on her grocery list but not for the last one or two impulse purchases that she does while in line at the cashier. Placing healthy food on such easily reachable locations - a form of nudging - is a way of correcting the wrongful use of heuristics, i.e. of biases, in situations where they lead to irrational decisions.

Applying this to Primary Care Plus, it becomes clear how the above interactions can be seen as nudging. During meetings, presentations and interviews, professionals involved in the organization of Primary Care Plus could often be found to use the following logic. In a hospital situation, patients as well as doctors have the tendency to (expect the medical specialist to) go to the bottom of any medical problem. This might be an effective heuristic when dealing with serious, life-threatening issues, but not in case of low-complex medical problems. Patients with low complex problems do not benefit from long and unnecessary medical trajectories. Also, they make the health care system needlessly expensive and inefficient. Relocating such patients to a primary care setting will make them adapt their expectations. In the case of the above consultation, the patient goes home on her own initiative without ordering any of the tests that the rheumatologist suggested. She is content with not more than some advice on how to "use her body" and how to "improve her posture". She knows now that she does not have RA, but besides that, the specialist had 
merely speculated on one or two other explanations of her symptoms. From the perspective of nudging theory, it seems that the conservative decision to wait and see instead of going for all the trimmings came easy because of the setting tempering her expectations.

In the second chapter, I identified the claim that relocating patients with low complex problems to a primary care setting will make health care more cost-efficient as the third promise of Primary Care Plus and as a form of nudging. I presented the most-often raised point of critique on nudging in general or on using nudging in a health care setting: Nudging is not legitimate, because it is an instrument of directing behavior that bypasses the rational capacities of nudgees. This makes it a form of manipulation that is detrimental to the autonomy of doctors, patients or any other nudgee. Subsequently, I explained the two arguments that proponents of nudging use to refute this point of critique: A nudge is merely a nudge, there is no force to it, and a nudge merely assists nudgees to make choices "that will make them better off, as judged by themselves" (Thaler \& Sunstein, 2008, p. 5).

Analyzing the back and forth between critics and proponents of nudging in the second chapter, I distilled two important dualities that structure nudging theory: duality of agency and the duality of design and action. First of all, as explained in chapter two, by denoting the theory "dual process theory", Kahneman (2011) presents the two processes of agency as separated from each other. While the situated processes of our automatic system on the one hand are immediate responses to the environment, the abstract processes of our deliberative system on the other hand produce "true" preferences, that is, stable outcomes that are independent of time and context.

The schematic understanding of action in nudging theory is problematic for two reasons. First of all, the situationism of automatic action in this schematic understanding of what it is to act is rather limited. This can be illustrated by the example of a nudge that Thaler and Sunstein (2008) propose for increasing the amount of donor registrations in the US. Currently, for a citizen of the US to be an organ donor after passing away, she needs to register. Because with such a policy, active steps are required, the number of registered donors in the US is much lower than the number of people who indicate in surveys to support donation (inertia bias). ${ }^{38}$ Thaler and Sunstein propose another policy, "mandated choice", in which everyone who applies for a drivers' license is mandated to check a box indicating their preference. The choice architecture in the nudge is the drivers' license application form. Changing the form is a way of putting the situatedness of automatic actions to work in

38 In a survey study conducted in $2019,90 \%$ of the respondents indicated to support organ donation, but only $50 \%$ had actually registered as a donor (HRSA, 2020). 
order to correct the inertia bias. While it seems impossible to make potential donors take active steps to register through providing information, it is much easier to have them automatically fill in a check box when prompted with the question in a form they are required to fill in at some point anyways. A nudge thus makes use of the possibilities and effects of changes in the context in which people make choices. The problem is that they do so in a very limited way. The situational cue of concern in the design of this nudge is merely the presence of the question and check boxes in the form, but other potentially relevant contextual factors stay out of view: the stress caused by the long waiting lines at the Department of Motor Vehicles (DMV), for example, the possibly hostile or eager behavior of the DMV personnel, feelings of being taken by surprise that potentially supportive organ donors might have when being prompted with such a heavy question in the routine-like process of applying for a driver's license (Whyte, Selinger, Caplan, \& Sadowski, 2012). These factors might all be of influence on the choice that potential donors make in the modified choice context.

The situatedness of automatic actions in the context of nudging theory is selective, i.e. there are one or two easily identifiable cues in the environment, while the possible effects of other aspects are not taken into account (Selinger \& Whyte, 2010). Applying this to the above observations, viewing the rheumatology consultation from the schematic understanding of agency in nudging theory, the conservative decision to wait and see is then enabled by the effects of patient and specialist being in a primary care setting instead of the hospital, where they have easy access to expensive diagnostic procedures. Holding this consultation to be the result of a successful nudge, other possibly relevant aspects of the socio-material environment in which patient and specialist do or do not achieve a durable decision are taken out of view.

The second problem pertains to the duality of design and action, which separates the nudger from the nudgee, and the act of designing a nudge from its implementation. While a nudger designs the choice architecture deliberatively, the nudgee makes situated choices without thinking. Lodge et al. (2016, p. 150) call this the "fundamental paradox at the heart of nudge": While choice architects base their interventions on the idea that the rationality of humans is fundamentally bounded, there is very little space for reflection on their own limitations in this regard. In the case of Primary Care Plus, the nudgers are the initiators, the partners of Blue Care who together came up with the idea of relocating low complex issues to a primary care location. The nudgees are the patients and medical specialists whose automatic choices, or at least their tendencies, are nudged within the environment to which the consultations are relocated. 
What the above problems show, as also argued in the second chapter, is that nudging theory, in its understanding of knowledge and morality, is phrased in a representational idiom. In terms of knowledge, a duality is implied between the nudger and the nudgee. The former resides in the realm of knowledge and possesses (scientific) information about how the latter act in the realm of action. Nudging's notion of morality has a similar structure. The schematic understanding of how people act (realm of action) on the one hand and what they truly prefer on the other (realm of morality) implies a representational structure as well. Nudging is then the activity of increasing the coherence between what people truly want and what they actually do, i.e. nudging is a way of helping them stick to their actual preferences. Again, such a representational notion of morality is crucial for the validity of the theory and the legitimacy of nudging as a policy tool, because only with such a duality of selves (the automatic and the true) nudging is not detrimental to autonomy but of assistance to it.

Using the representational idiom in order to assess nudging in the above observations renders the consultation into a clear-cut choice between a wrong and a right option and it establishes a causal relation between the environmental cues and the outcome of the consultation. Retelling the above observations in nudging template, the patient's and specialist's assumed stable preference is to not take a treatment decision that is redundant from a medical point of view, which, from the organizational viewpoint, is the rational preference for patients to have. Holding the consultation in a primary care environment instead of a hospital assists specialist and patient to stick to this preference. When rendered as such, the above observations fit the narrative style used for discussing the mechanism of nudging. In such stories, nudging is seen as an intervention in a clear-cut decision process, in which the right choice (take the stairs, wash hands, healthy food choice) can be unambiguously discerned from the wrong one (take the elevator, skip washing hands, unhealthy food choice).

Such a rendering is problematic, because it does not do justice to the decision making of the above rheumatology consultation for two reasons. First of all, Primary Care Plus caters to patients with low-complex health problems, such as the rheumatology patient above. While the patient's problem is of low complexity in a medical-technical sense, it is not in any other sense. In this case, there is quite some ambiguity whether further testing is needed or not. A doctor starts from the medical issue as the patient experiences it, without necessarily having or obtaining a precise idea of the underlying disorder. But even if they know or find out during the consultation, the distinction between a right and a wrong treatment decision still depends on the severity or stage of the disorder but also on many social or personal 
issues that can often not be disentangled from the medical-cognitive aspects of the problem. Even though it would be best for everybody if we do not make wasteful decisions in health care, distinguishing a needlessly expensive decision from a necessary one is not as obvious as deciding to wash hands or not.

In this chapter, I take up the performative instead of the representative idiom to enable a more thorough ethical assessment of how nudging turns out in the rich practice of Primary Care Plus. In essence, this means that the choice architecture, i.e. the primary care environment in the context of the study, is just one of the emerging agencies that needs to be navigated in order to achieve a treatment decision in the mangle of practice. Some of the agencies might be given beforehand, but others come up in the interaction of patient and doctor with each other and with the material environment. To illustrate, Whyte et al. (2012), also mentioned above, have taken up a similar approach when criticizing the nudge of mandated choice. In a general sense, they argue that by proposing the nudge of mandated choice, Thaler and Sunstein fail to take the meanings people attribute to (aspects of) choice contexts into account. Preferences of people might change when having to make such a choice quickly amid the stress and frustration of long waiting times, opiniated personnel and the pressure to quickly acquire a license.

In the previous chapters, the performative idiom proved useful for bringing the dynamic work processes into view, by which firstly managers and doctors, and secondly doctors and patients, navigate the mangle of practice in order to enable smooth delivery of Primary Care Plus. Building on Strauss' $(1985,1988)$ notion of articulation work, the mundane, informal and often invisible activities involved in making organizational processes run fluently through joining the distinct parts, I have developed a knowledge dimension of articulation work, knowledge work, and a moral dimension, justification work, which I substantiated with Boltanski and Thévenot's (2006) orders of worth. Justification work, I explained in chapter four, is the seemingly mundane work of aligning the moral orders of worth in the consultation in order to achieve a durable treatment decision.

In this chapter, I will use this notion of justification work again to analyze Primary Care Plus consultations as mangles of practice, in which patient and medical specialist navigate emerging agencies and align moral worlds in order to achieve a more or less durable treatment decision. By bringing the rich and complex dynamic processes unfolding in the consultation into view, I aim to ethically assess how nudging turns out in the practice of Primary Care Plus. What does the practice of achieving treatment decisions in Primary Care Plus entail? How is the nudge navigated in practice? Can the condition of legitimacy still be satisfied when the right option is not so easily discerned from the wrong one? I will thus answer the question 
whether Primary Care Plus as a nudge is legitimate in a health care setting.

In order to answer the main question of the chapter, I will track justification work conducted in three consultations: the above rheumatology consultation, the internal medicine consultation already analyzed in the previous chapter and one of the ophthalmology consultations from the previous chapter as well. I also use an excerpt of observations of a management meeting at the orthopedics department at the hospital about their Primary Care Plus consultation hours at at the center. On the basis of these three analyses, I ethically assess how nudging is put to work in Primary Care Plus. To give a short preview, on the basis of the analysis of the ethnographic observations, I will make three arguments. The first pertains to the first duality identified in nudging theory, the second to the duality of nudger and nudgee. The third is concerned with the question how intervening in a choice architecture can be legitimate, departing from the reflections on the four examples of the chapter.

\section{INTELLIGENT ROUTINES}

As explained above, an important building block for nudging theory is the duality of agency. People either act deliberatively or automatically. For the legitimacy of the nudge, the question whether it is detrimental for the nudgee's autonomy is crucial. Understanding the processes of decision making of doctor and patient in the consultation room, while supposedly being nudged, is thus crucial when assessing whether or not it is legitimate to use such a policy tool in this context. As viewed from the nudging rationale, the above described rheumatology consultation can be interpreted as an immediate response to the choice architecture: In the primary care setting of the Primary Care Plus consultation, it comes naturally for patient and specialist to take the conservative decision to wait and see. The patient and the specialist have thus, without much thinking, been successfully nudged towards deciding what is best. In an analysis built on the nudging rationale, her automatic process of taking the conservative decision is devoid of reasoning.

Adopting the performative idiom instead yields a contrasting account of the consultation. As argued in the previous chapter and above, besides collecting medical information, making up a diagnosis and communicating the options, doctor and patient are also involved in a process of justification work, attempting to achieve a more or less durable treatment decision. What stands out in this regard is that the patient, before coming to the Primary Care Plus center, felt not taken seriously by her general practitioner, because he had been unwilling to go to the bottom of her problem. That the patient had pushed to go to a medical specialist shows the initial treatment decision of general practitioner to not be durable. At the Primary Care Plus center, the patient does leave the consultation room content, even though the 
rheumatologist at the Primary Care Plus center merely analyzed the older results and $\mathrm{x}$-ray images and physically examined her without finding out much more than the general practitioner had. Following the findings of the previous chapter, in order to understand the consultation, taking outcomes into account is not sufficient; it is crucial to bring the interactions by which durability was reached into view, i.e. the justification work conducted during the consultation. The following excerpt gives a more detailed account of a crucial part of the same consultation:

After the physical examination, the patient and the specialist sit down at the desk again. Specialist: "Your general practitioner has ordered tests for inflammation markers in 2011 and in 2013. It all looked good. Let's see if I can find the x-rays." She turns the computer screen towards the patient.

While describing out loud what she sees, she constantly points to different parts of the image on the screen. "We are looking now at the shape of the hips. Do the different parts fit well? As you can see the joints are beautiful and smooth. When we conduct these kinds of examinations, we closely look, for example, at whether the edges of the joints are frayed. On this picture we do not see that at all."

The $x$-ray image of a hand appears on the screen. "This is the thumb, these are the finger bones, (...). Here as well, I check whether everything is nice and smooth. As you see, there are no scores or little pieces of bone missing." She points at a specific part of the hand and says: "Here, it looks very nice as well!"

Next is an x-ray image of the patient's upper body. "The vertebras of your spine are beautifully aligned. The distances between each of them is similar. If you would change your posture, which is somewhat hollow, you could possibly take away quite a bit of pain." The rheumatologist gets up from her chair to show her how to correct a bad posture: "Bend the knees lightly, tuck under your pelvis", she says while illustrating it by doing it herself. Then: "None of the diagnostics that we have right now show abnormalities."

While these interactions seem a straightforward example of a doctor informing a patient about her medical condition, the patient's own interpretation of the exchange suggests something else. When asked afterwards what made her leave the consultation content, she says:

the conversation [with the specialist] was very pleasant. I was happy she checked everything, and also that she wrote it down and filed it in the computer. (...) Her examinations were more extensive [than those of the general practitioner] (...) and it made a difference that I have seen it myself now. (...) Not like 'Oh, that looks good', click, 
click [mimicking the general practitioner quickly clicking through the $\mathrm{x}$-rays]. (...) She said, 'when it looks smooth, there is nothing wrong.' And then, I saw for myself that it looked smooth, that is why I feel confident about it.

The two excerpts show that for the patient not merely the content of the interactions is leading but also the way in which the specialist shares it. For the patient, as became clear in the interview beforehand, it is crucial to be taken seriously. Going through the detailed observations of the above and her reflections on it shows that the need to be taken seriously is a processual concern. It is not about getting more tests; it is about being involved in the decision-making process, and to not be set aside by a doctor who "click-clicks" through the photos. In the way in which the rheumatologist goes through the images with her, the patient becomes an autonomous agent, who is not merely as knowledgeable as the doctor in terms of what the $\mathrm{x}$-rays show but also in how to deduce the right conclusion from it.

The analysis of the rheumatology consultation shows three important aspects of the justification work of concern in the chapter. First, the justification work conducted by the rheumatologist entails shaping a decision-making interaction in which they would be equals, i.e. the rheumatologists succeeds in tuning into the patient's civic order of worth. Secondly, autonomy of the patient is not given beforehand but emerges in the interactions between patient and doctor. Last, the justification work does not merely cover the span of the consultation, but it is a process that extends before and goes beyond it. It is the work done in the consultation room itself to tune in or allow for tuning into the patients order of worth, but also the work done by the patient, anticipating on the consultation coming up and reflecting on it afterwards.

If we now go back to the duality of agency, an important contrast becomes clear. Even though it is possible that the patient's decision to wait and see was not preceded by a reasoning process, the justification work she conducts afterwards shows the interactions with the doctor are logically related to her ideas of what is right and wrong in taking a treatment decision together. A first, preliminary conclusion can thus be drawn. Even though her decision in the consultation to wait and see came unexpectedly and quickly, possibly automatically, this does not mean that her choice was not informed by reasons.

To further elaborate on this preliminary conclusion, it is important to get more clarity about what it means to act for reasons. To enable explicating the differences between the nudging account in the introduction and the performative-idiom-account in regards to reasoned actions, I will contrast the dual process theory with theories about reason and action in moral philosophy. The dual process theory was developed by Kahneman (2011) in the 1980s as a critical alternative to the then 
dominant rational actor theory. Many experiments in the behavioral, cognitive and neuroscience had shown that it is exceptional for people's actions to be preceded by processes of deliberation. The dual process theory promised to offer a more realistic model of agency, thus offering a better point of departure for explaining and predicting behavior. Other theories of agency were developed as a response to the same findings with a significantly different conception of what it means to act for reasons. On the basis of the work of moral philosophers such as Pettit (2007) and Velleman $(1992)$, Sie $(2012,2014)$ has proposed what she calls the "traffic participation view" on human agency. When we drive a car or ride a bike, most of our decisions are taken automatically, as a response to cues from traffic and traffic signs around us. We do not think before turning on the indicator, nor when we stop in front of a traffic light or pull up when the light turns green. We act and interact with other traffic participants without much effort or consideration. When we get into an accident, however, we reconstruct reasons for our decisions with hindsight. Sie thus distinguishes two modes of agency: the "post-crash agency", the active process of reconstructing reasons after a crash, and "the participatory nature of agency", i.e. the effortless actions taken as a response to social and material cues in the environment (Sie, 2014, p. 273).

In contrast to the dual process theory, the traffic participation view takes effortless actions to unfold in an ongoing process of being informed by reasons. ${ }^{39}$ Applying this to the rheumatology consultation, when the patient afterwards explains why she took the decision to wait and see, she reconstructs her reasons with hindsight. Combining Sie with the earlier introduced process of exnovation, in which it is important to make visible how things are done well, it is not only in the aftermath of a crash or a crisis that actors reflect afterwards on their automatic actions, but this could also be done when a consultation ran smoothly. ${ }^{40}$

\footnotetext{
39 This is a subtle difference from the dual process theory. Following biases is also a form of action "informed by reason". The difference is that the process of being informed by reason, in the dual process theory, is finalized. In that sense, biases are fossilized. In the traffic participation view, effortless actions are part of an ongoing process of evaluation and adaption to moral norms and convictions.

40 During the ethnographic research it stood out that many patients are not very well able to explain what made them satisfied with the care they received. It was especially the ones that had negative experiences in the past and recounted those during the conversation that were able to disclose what they liked about it. This resonates with Sie (2014, p. 281), who writes: "We might or might not be aware of the features that determine our actions. Whether we are, is partly determined by the prior attentive aspects of our agency to the 'crashes' we suffered (e.g. the conflicts and disappointments we came across, the mistakes and failures we suffered and so on) and the deliberations that were needed to be able to interact fluently again."
} 
Some behavioral psychologists downplay the importance of such processes of reasoning with hindsight, because, according to them, they are mere confabulations (Haidt, 2001), i.e. they do not have any significance as an indication of our true motivations for the choices made or the action done. But even if they do not give access to our true motivations, Sie argues, this does not mean they are not of crucial importance to us as moral and social beings. Reconstructing the reasons for our automatic actions afterwards (with others) helps us navigate the social world: building relationships, clarifying for ourselves and to others which actions in what situation for what motivations are morally acceptable and which are not. The activity of reflecting on our actions shapes the ways we will respond to similar situations in the future. They do not give us introspective access to the patient's "true" motivations; rather, they help us understand how she makes sense of her actions as part of a larger, more or less coherent framework indispensable for effortlessly participating in the social practices that make up our daily lives. We often act without thinking, but we are still "reason-responsive-beings" (Pettit, 2007). Our actions are related to reasons, though not always in a causal linear way. While the dual process theory researchers depart from a conception of action as a momentary choice, which is or is not preceded by a process of reasoning, this model understands the relation between reasoning and acting as a process stretching over time, unfolding in practice and between past and future events.

Note that the difference between the value Sie and Haidt consider confabulation to have is related to the idiom in which they phrased their theories of moral agency. In Haidt's argument, set in a representational idiom, the justification of an action, i.e. moral reflection on the action afterwards, is only of value if it gives us access to what the actor's actual reason has been. He thus separates the knowledge realm, in which the reflections reside, from the realm of action, in which the actions are conducted. The knowledge realm is only of value as a representation of what is truly the case in the world out there, the realm of action. Sie, on the other hand, takes the reflections itself as part of one and the same realm as the automatic actions that are its subject. Such reflections are performative; they shape the agent's moral agency. Both reflections and actions are part of one and the same process in which people navigate through and engage with the world, in which reflections and actions mutually shape each other. Her argument is thus set in the performative idiom.

If we apply Sie's insights to the patient's explanation of her decision to refrain from tests, we can conclude that although her decision might have come quickly and without much thinking on the spot, it fits a larger more or less consistent framework of reasons. The patient had been worried because of her unexplained symptoms, her mother's story about her 26-year-old colleague who fell fatally ill fed those worries, 
and she felt misunderstood, because the general practitioner treated her in a way that clashed with the moral order of worth she deems appropriate in the consultation room. That the worrisome feeling disappeared with the justification work of the specialist - making her an equal participant in the process of reading the $\mathrm{x}$-rays and drawing conclusions, aligned with the civic order of worth she deems appropriate - only makes sense if reasoning is taken as a non-linear process that extends beyond the consultation itself. The decision to wait and see was made in a split second, probably not preceded by a rational process of weighing the options, but that does not make it less reasonable. As a process of reconstructing what happened on the spot, the logical puzzle of reasons, actions, experiences and emotions as a representation of her reasons has an important formative effect on future actions.

Further combining Sie's view on agency with the moral dimension of articulation work, we can see that we should understand deliberations not only as having a function in our deliberative system of acting but as thoroughly connected to developing competence in taking automatic actions. Similarly, justification work is not just momentary tinkering in search of a sufficient alignment; it has consequences for how patient and doctor will navigate similar situations in the future. Justification work, conducted with others or alone, help us make sense of what we did, and are thus indispensable for improving future decisions. For the patient, it is part of learning to decide on the spot and to be able to change the direction of such immediate and automatic actions when prompted with unexpected interactions. Learning a skill is not a matter of just appropriating the content of a skill in a void, for example by reading a book with instructions on how to make decisions in a logical fashion, nor is it a matter of merely doing it over and over again without thinking about it; Instead, it is a process in which the actor goes back and forth between reasoning about what she thinks she should be doing in the situation and what she has actually done and trying out what works best every time. Such a process of learning is ongoing. The activities we end up conducting and how we justify them to ourselves and others shape each other, making it impossible to determine whether an action is automatic or the result of a justification or reasoning process. In this light, the duality of an automatic system, which can be easily directed by changing the environment, and a rational system, as a producer of stable true preferences, seems to be an unconvincing caricature of what it is to act.

From the two main points that I made in the above analysis of the rheumatology consultation, I can draw one important conclusion. The first point is that the autonomy of the patient, an especially important value in health care settings, is not something that a patient possesses and that should be protected in the course of the consultation, but it is a characteristic that emerges in interactions. In this 
case, the patient's discontent with earlier received care was a consequence of the feeling she had not been taken seriously. By taking her step-by-step through the process of diagnosis and decision-making, the specialist tuned into the civic order of worth that the patient deems appropriate, making space for autonomy and equality to emerge in the consultation room. Second, the quick and impulsive action to wait and see, understood in a performative idiom, should nevertheless be seen as informed by reasons. After all, the patient actively placed the actions in a moral, somewhat coherent framework of reasons that starts before and extends beyond the timespan and setting of the consultation itself. From this, I draw the conclusion that such effortless - automatic - acting is thus a form of habitual behavior, not in Kahneman's sense of the word but in Dewey's, introduced in the first chapter. For Dewey (1922), a habit is an "acquired predisposition" that makes us sensitive and connected to certain stimuli, preferences and aversions. Acquiring such predispositions is crucial, because they are our most important instrument through which we engage and thus also perceive, imagine and reason with the world around us (Iedema et al., 2019). Within Dewey's pragmatic approach to action, these predispositions are not biases that yield automatic - mindless - responses to cues in the environment, but they are intelligent routines in an ongoing process of being shaped by reason.

\section{SITUATIONISM MEDIATED BY MEANING}

In the previous section, I have focused on the assumption of the dual process theory that automatic actions are devoid of reasons. Another assumption that follows from the duality of agency in nudging theory is that actions are either informed by reason or situated. By scrutinizing one of the consultations described in the previous chapter, the second ophthalmology consultation, I will propose an alternative understanding of the reasonedness and situatedness of actions.

The argument of this section is based on the analysis of the second ophthalmology consultation of the previous chapter. The patient made an appointment at the Primary Care Plus center because her optometrist could not get her prescription glasses right. During the examination, the ophthalmologist found an early phase of macular degeneration. As explained in the previous chapter, whenever he detects any phase of macular degeneration in a consultation at the new high-tech eye clinic in the hospital, it is part of the work routine to order an OCT. At the center, however, the routine decision to do an OCT is not the most likely trajectory when the medical problem is found in such an early phase, because it involves making another appointment at the eye clinic and making the effort to see a specialist again. The treatment decision here, too, is to not do anything while in the early phase of the 
illness. At the end of the consultation, the eye doctor tells the patient to keep an eye on whether her sight is deteriorating by regularly looking at the lines between the bathroom tiles, and to come back when they start meandering.

Applying the nudging template renders the decision making that the nudge has interfered in into a clear-cut choice between ordering an OCT or not. The rational option would be to not order it, because in such an early phase of macular degeneration, a diagnostic procedure is not strictly necessary from a medical point of view. The absence of high-tech diagnostic procedures in the Primary Care Plus center assists patient and specialist to stick to the wiser choice. This would have been difficult in the hospital, because there, making an OCT is a standard, i.e. automatic, procedure. As seen from the nudging framework, what counts as the rational option, to not order and OCT in case of an early phase of macular degeneration, is stable. The question into its rationality can be viewed in isolation from any aspects of the context, and is stable regardless of the changes in the choice architecture.

In the previous chapter, I also analyzed the ophthalmology consultation with a performative idiom. This brought into view that in the specific case of the patient with an early phase macular degeneration, the relocation to a low-tech setting did not just increase the patient-centeredness of the care delivered, and in the context of this chapter, it does not just assist patient and specialist in taking the most cost-effciency decision; it also causes a shift in what the eye doctor deems the appropriate moral order of worth in the situation. When seeing patients in the high-tech eye clinic at the hospital, it is natural for the ophthalmologist to follow the industrial-market order of worth inscribed in how the workflow of the ophthalmology outpatient clinic is organized. This workflow is designed out of an anticipation of what would make patients-as-consumers satisfied, expecting them to have scarce time and thusto prefer to be helped in an efficient manner without spending too much time sitting in waiting rooms. There, he does not feel the need to take anything else into consideration when choosing whether to order an OCT or not but the patient's medical condition - he just follows protocol. Now, at the Primary Care Plus center, suddenly he finds himself in another order of worth in which it is of concern to take into account how well the patient is able to come to a second consultation at a different location. He needs to think for and with the patient, not only about her medical needs but also her needs in terms of her mobility and the availability of help. He feels pressured to take up the role of a doctor as shaped in the domestic order of worth.

To understand how the above performative-idiom-analysis contrasts with the nudging account of this consultation, and to be able to track and analyze the doctor's processes of moral reasoning, I add Sie's view on human agency here. 
I highlight two points of contrast here. First of all, a performative-idiom-approach shows that the process of achieving a treatment decision is situated in a less limited way than the situatedness of actions in nudging theory. As explained above, with the traffic participation view, Sie (2014) differentiates between two modes of agency: the "post-crash agency" and "the participatory nature of agency". Taking a decision together in the consultation fits the second form of agency. It is a participative process, in which the doctor has become skilled over time. As argued above, it is a reasoned action when not taken as a momentary decision but one that is connected to past and future. The ophthalmologist quickly perceives the medical problem and the many other contextual factors, such as the conditions in which the patient had to come to the center. Such a train of perceptions, followed by a seemingly effortless action or decision, is an interpretative process. As we have seen above, how the doctor understands the contextual factors, and how he prioritizes concerns and, thus, how he responds to them, depends on his past experiences and on how he has logically related them to an overarching framework of reasons. In the consultation, the effort that the patient would have to make to get an OCT became more decisive than the desire to give optimal care. The highest common good went from efficiency for the hospital and the patient-as-consumer's satisfaction (industrial-market) to comfort for the patient as a person (domestic-industrial), whom the doctor feels responsible for as if she were his child. This, however, could be otherwise, depending on (his understanding of) past experiences in dealing with early phases of macular degeneration and his convictions of which moral orders of worth should be appropriate when.

On the drawing table, the relocation of Primary Care Plus was hypothesized to have an effect because the setting would change the expectations and demands of patients and the mindset of specialists. Here and in the previous chapter, we have seen that the relocation also has another effect, depending on how the doctor interprets the situation. Adding to the analysis of the previous chapter, however, it has become clear now that this relocation makes the specialist have to take considerations into account that he thinks should be of no concern. The meaning of ordering an OCT depends on the context and the appropriate order of worth. Actions are often automatic and situated, which makes the relocation and the accompanying change of meaning have important consequences. What a doctor decides automatically depends on the meanings he attributes to the material and social context in which he acts. How such changes of meaning unfold and,thus, how they affect the automatic decision making is hard to predict, because these situated actions are mediated by meaning.

The above leads to two conclusions. First of all, both in Kahneman's dual pro- 
cess theory and in Sie's participation view of agency, actions are situated. Especially when in the participative mode or when using the automatic process of acting, agents' actions are immediate responses to the environment. The crucial difference, however, is that while in Kahneman's theory, a causal chain is assumed between preselected and limited environmental cues on the one hand and the actions of an actor without any specific preferences or experiences on the other, in Sie's theory, the way in which an agent responds to the environment depends on what she has learned in the past, her moral convictions and what meanings she attributes to the context. Combined with the performative idiom and the context of the study, how actors navigate the emerging agency of absent apparatuses depends on what moral order of worth they deem appropriate, i.e. such situated responses are mediated by meaning. The second aspect worth emphasizing is that, again, what is deemed good care for the ophthalmologist is not a stable given; it is different in the eye clinic, where it needs to be optimal, and different again in the Primary Care Plus Center, where the doctor assumes a patient does not want to make the effort again to go to another consultation. Preferences, that is to say, convictions of what good care entails in regards to one and the same medical condition, are not stable but emerge in the context in which care is delivered.

\section{NUDGING AS AN INTERVENTION THAT PROMOTES SCAFFOLDING}

In the previous two sections, I reformulated two aspects of the duality of agency. While in the dual process theory, it is assumed that the two agency processes are separate, I showed that automatic reasons are at least in two ways informed by reasoning. First of all, they are in an ongoing process of being placed in a somewhat coherent moral framework in which actors learn what they value and how to reason in the world and secondly, the situatedness of automatic actions is mediated by reasons. In this section, I will further elaborate on the important role that automatic, or effortless, actions play in learning processes by going back to the internal medicine consultation of the previous chapter. In this consultation, a male patient came in with many different symptoms and fears, bowel problems and IgA deficiency. Before the consultation, the patient complained that he was referred to a different medical specialist than the one he usually visits in the hospital. Nevertheless, he left the consultation room content, even though they did not decide to order tests or see the specialist in the hospital, or find an answer to the question of what is causing his symptoms.

Viewed from the nudging template, this consultation shows a successful nudge as well. If we take the decision making of the specialist and patient to be automatic, the context only matters insofar as it is part of the designated choice architecture. We 
then understand the specialist pushing for a conservative decision and the patient accepting it fairly easily as an immediate adaption to the low-facility environment. The treatment decision becomes a choice between two clear-cut options, to refer or not, of which one is presented as the obviously wiser one.

Adapting the performative idiom in the previous chapter, I gave a different account of what happened then and there in the previous chapter. I argued that the slow and careful development of a Primary Care Plus routine is an instance of justification work in which the specialist, by continuously dealing with patients with similar problems and anticipating what they need and what she has done, develops a routine shaped by the domestic order of worth, which is aimed at helping patients restore trust. This is comparable to the process described above when analyzing the rheumatology consultation. The process of developing such an intelligent routine, in this case for dealing with a specific kind of patient with broad trust issues, is like placing the singular instance of a consultation in a moral framework once again, lifting it to a more general level. Following Sie (2014) and building on the argument about the rheumatology consultation, we could say that the Primary Care Plus center gave the specialist the space and flexibility to go back and forth between ideas about how to improve care for these specific kinds of patients and to try them out in practice, i.e. to develop and master the skill of effectively helping patients with such problems.

When viewing the decision making in Primary Care Plus consultation as such, a new and possibly more productive understanding of how intentionally changing the choice architecture of health care delivery can be morally responsible. In the case of the consultation for internal medicine, taking the decision maker out of her usual setting, and thus her routine, made her reflect on what she does in her daily work for what reasons, opening up space to try out new ways of dealing with some of the problems that many patients have. The changes in the choice architecture, i.e. the relocation of specialist care, had been effective, not because the automatic mindless behavior of the specialist had been redirected but because she was taken out of the mindlessness of doing her daily job. This type of changing the choice architecture can then be understood not as a bypassing of rational capacities, such as most nudges do, but a way of turning such capacities on. It became clear in the analysis of the consultation for internal medicine that processes of reasoning are breeding grounds for new ideas. How do strategies work out, what word choices, what decisions turn out for the best for which type of patient? If we see that reasons and actions do not necessarily have a causal and linear relation to be significant, we can also see that it is not the effect on mindless behavior that makes changing the choice environment an interesting tool but, in contrast, the effect on the content 
and creativity of the reasoning processes. Note here that a different and alternative notion of the experimental nature of the Primary Care Plus intervention, described in the introduction as a controlled trial, is required to even notice these beneficial effects. Understanding the experiment as a procedure for creating new phenomena instead of a controlled trial assessed with outcome measures allows for noting such unexpected improvisations and surprising outcomes that are otherwise rendered invisible (Hacking, 1983; Zuiderent-Jerak, 2015).

An important insight in moral philosophy is that moral agency is not an inherent characteristic of human beings but that it emerges from a process of establishing contextual and normative relations with the world around us (Vargas, 2013). This resonates with the above observation that autonomy, as a crucial part of moral agency, is not something that an agent possesses and that should be protected but that emerges in interactions. In moral philosophy, the practice of doing, discussing and cultivating moral agency is denoted as a process of "moral scaffolding" (Milam, 2018). Although announced as a way of "seducing" specialists to do the right thing, taking the specialist in internal medicine out of her daily routine enhanced her capacity as an agent to come up with and try out new ideas for solving the problem of patients getting stuck in long hospital trajectories. While Primary Care Plus is presented as a limited or conservative form of specialist care, in this case, it becomes clear that in other respects, it also extends the doctor's possibilities. In comparison to her work in the hospital, here, she has time, space and flexibility to further develop ways of solving what she sees as one of the most pressing problems in health care of today. As such, it strengthened her moral scaffolding. When we see Primary Care Plus as an intervention in an environment in which decisions are ongoing processes, in which reasoning and automatic acting are intertwined, they make for an interesting option for innovating the delivery of health care.

\section{REFLECTIONS ON THE DUALITY OF AGENCY}

In the above section, it became clear that the most important two differences between the dual process theory as used in nudging and the traffic participation view of human agency are the assumptions concerning rationalism and situationism. While in nudging theory, rational actions are only those exceptional actions that are preceded by a careful weighing of the options, in the traffic participation view, reasoning processes are seen as crucial for learning how to act automatically and for building an overarching framework of reasoning in which automatic actions make sense. Situationism in nudging theory means most of our actions are immediate responses to one or two easily identifiable cues in the environment. Combining the performative idiom with Sie's notion of agency, automatic actions are responses 
to the environment as well, but how an actor responds to the numerous, sometimes indiscernible aspects of the context, depends on her experiences in the past, what those aspects mean to her and what she thinks is the right behavior in such situations, i.e. the response is mediated by meaning.

Explicating such differences is important, because they shape our expectations of such an intervention and the way we implement, evaluate and try to improve it over time. When the decision making in the rheumatology consultation is stylized as a nudge for example, it becomes an engineering input-output model. The input consists of the patient's problem (painful joints) and the limitations of the primary care setting itself; the output is then the conservative decision to wait and see. The reasons that a patient or a doctor might have or might give when asked are irrelevant for understanding what happened in the consultation room. The ophthalmology and the internal medicine consultations can be accounted for with a similar structure. The patient's medical problems and the limitations of the Primary Care Plus setting make the specialists send their patients back with merely an advice for the general practitioner. The context in which the specialists take decisions is only of importance in a specific way: as a gentle push towards conservative decision making.

Understanding the consultations as such stimulus response models has two important effects. First of all, such a conceptualization comes with a promise of predictability and control. From the start of the project, initiators presented a hypothesis of how effective such a relocation would be (prediction) on the basis of earlier scientific research (Schulpen, 2003). If the output deviated from the hypothesis, it would be a matter of engineering the input to improve the output (control). This is typical for how managers of the organization evaluated the performance of the center and discussed possible solutions for its problems. During meetings with heads of departments, or during the users' council meetings with general practitioners and medical specialists, typically, one of the project managers would show slides with bar charts that give an overview of the referral rates, which functioned as the indication of how specialties or individual medical specialists were performing in the Primary Care Plus center (output). The referral rate is the percentage of referrals to a secondary care setting. As long as a group of specialists from one specialty on average had a lower referral rate than the hypothesized $25 \%$, delivering such specialty care is deemed successful in Primary Care Plus. During management meetings and in users' council meetings as discussed in the third chapter in which deviations were discussed, the problem was often seen as caused by general practitioners sending the 'wrong' patients to the Primary Care Plus center. Framing the problem as such, the indication lists that came up as an important topic of discussion in the third chapter became the appropriate solution. To rephrase, because Primary Care Plus 
as nudge is implemented and evaluated as an input-output machine, it is only natural that the most important means of improving the output is to change the input by informing the general practitioners which medical problems should be sent there an which should not (indication lists).

Secondly, when a nudge is stylized as an input-output machine, how the process unfolds, what happens between specialist and patient during the consultation, stays blackboxed. Also, other factors outside of the consultation room, in the spatial and temporal sense of the word, stay out of view as well. Bringing what happened there and then into view is a process of exnovation, I explained in the chapter one. This is what I have done above. In the three consultations that I analyzed in detail, different, more diverse accounts of how good care was delivered in Primary Care Plus consultations became visible. Furthermore, a problem that would otherwise have stayed out of view came up, but also an opportunity of developing a new way of delivering health care that emerged with intentionally changing the choice architecture with an open end. Seeing how a nudge works in practice by opening up this black box, i.e. exnovation, gives us information that is crucial for improving such practices. The above has shown how the qualitative first-person accounts of people involved in decision making give us informative insight into the processes going on on the work floor and, thus, about how Primary Care Plus is done in practice.

\section{DUALITY OF DESIGN AND ACTION}

While in the previous sections, I addressed the first duality of nudging, in the remainder of the chapter, I will address the second one: the duality between nudger and nudgee. This second duality, I noted in the second chapter and in the above section, is aligned with the duality of knowledge and action. Nudging theory deals with two kinds of actors: first, the nudger, the designer of a nudge, who carefully determines what is rational for the nudgee in the choice situation of concern and uses these insights to design a choice architecture that will increase the likelihood of people to make the rational choice in such a situation.; the other kind of actor is the nudgee. She is the person whose behavior is in need to be corrected because her rationality is bounded, i.e. there is a gap between what is best for her, as judged by herself, and what she actually does.

The management level of Primary Care Plus is of concern for its legitimacy, because this is where the decision environment is designed. As explained in the previous section, the design of a nudge is often based on scientific findings about how the choice context directs people's behavior, in the case of Primary Care Plus, on a research into joint consultation of general practitioners and rheumatologists (Schulpen, 2003). The research that Primary Care Plus is based on initially focused 
on improving the communication between professionals in primary and secondary health care, but along the way it was found that rheumatologists ordered fewer diagnostic tests and follow-up consultations for the same type of patients in the joint consultations than in the outpatient clinic of the hospital. In the discussion at the end of the article about these findings, the discrepancy is attributed, among other explanations, to the "expectations of patients towards the hospital" and "the routine [of the rheumatologist] at the outpatient clinic" (2003, p. 6). And in a more speculative tone, Schulpen states: "perhaps that the practical, primary care way of handling things, stimulated the specialists to use time and means critically" (2003, p. 6).

In literature about nudging, the implementation of a nudge is often taken to be an experiment by which the hypothesized effect is tested, such as in Bronchetti et al. (2013). Monitoring such interventions and writing a scientific article about it means determining whether the nudge works as an instrument of top-down behavioral change or not. This makes sense within nudging theory, which takes the situatedness as limited to a set of easily identified environmental cues, and the nudgee's actions as similarly easy to observe changes in behavior. This enables a reduction of the complex reality of action into a straightforward, causal relation between the cues in the environment and its effects and thus structures the events as controllable and, thus, easily tested. From this viewpoint, managing Primary Care Plus is an activity of monitoring the performance of the centers, taking these as an indication of how well the project is doing and which conditions (input) should be altered. In order to further explore how such an idea works out in the practice of managing and delivering Primary Care Plus, I will now turn to ethnographic observations of a meeting at the orthopedics department, in order to bring into view how such monitoring and managing was done there and then, i.e. I describe this monitoring and managing in the performative idiom.

The meeting at the orthopedics department of the hospital that I will analyze in this section took place in the Spring of 2015, when the centers had been offering consultations for around seven months. During this meeting, in which the daily manager of the project (M1), the director of the primary care organization (M2), a project manager of the hospital (M3), the head of the department (O1) and one of the orthopedists who works at the center (O2) attend. The meeting was set up to discuss the performance of the department's orthopedists at the center and the possible ways to improve it.

The M1 has placed her laptop in the middle of the table. The doctors and managers attending the meeting sit around the table, stare at the screen and listen. On the 
laptop, a slideshow presentation, consisting mainly of bar charts with management numbers, is running. After the numbers of the center as a whole and the specialisms separately, she proceeds to a bar chart consisting of eight charts in different tones of blue, showing the personal referral rates of different orthopedists. As soon as the chart appears on the screen it becomes quiet in the room. The $\mathrm{O} 2$ and the head of the department seem nervous, waiting to see how they are performing personally and as a department. (...) M1 explains: "When we look at each doctor's personal referral rate, we see considerable differences. Some of the orthopedists refer a big chunk of their patients to the hospital. Like Kees van Dongen.41 He has seen only eight patients, but he referred seven."

The moment when the manager speaks out the name of the specialist, the $\mathrm{O} 1$ and the $\mathrm{O} 2$ start sniggering, apparently thinking about how the numbers fit the impression they have of Van Dongen as a person. Not much more is said about it until the end of the meeting. O2: "As the numbers have showed us, not everybody is suitable for providing Primary Care Plus." (...) M1, agreeing with the orthopedist's remark: "You need to have the guts to make decisions on the basis of limited diagnostics." Concluding the discussion, M2 says: "Of course, if a specialist says, 'this is not my cup of tea,' I think there should be space for that. I rather have them be clear about that and not work here than that they do it reluctantly and send each patient to the hospital."

At the level of managing Primary Care Plus, the industrial logic is dominant. The performance of a specialist is evaluated by translating her decisions into a number, that is, into the so-called referral rate that is an expression of the specialist's efficiency in terms of how many patients she has referred to the outpatient clinic. Such a translation is an operation by which M1 lifts the individual decisions of a doctor to a general level at which they can be easily compared with that of others and with the hypothesized referral rate of $25 \%$. For the industrial rationale to be dominant is not surprising. Even though the research team also extensively monitors patient satisfaction and quality of care, for management it is important to immediately have an idea of how well the center is doing in terms of the goal of the project, to increase the cost-efficiency of health care.

A referral rate, however, is more than a passive representation of a state of affairs. Such an operation functions within a certain constellation. Thinking, speaking and acting within such a constellation creates a world with a specific hierarchy of types of people, actions and objects. Within a constellation, certain kinds of responses to the numbers come more naturally than others. Seen from the dominant industrial

41 The name of the orthopedist is changed for reasons of anonymity. 
logic, specialist van Dongen's decisions acquire the status of being irrational. His decision making is not a reason to inquire in his motivations, or to see how and why he decided as such, but a deviating element in world in which rationality is shaped by the industrial order of worth. His deviant behavior creates uneasiness, followed by the conclusion that he as a person is unsuitable for the project and that the project and himself would be better off if he did not participate.

O2 brings up another cause of the high referral rate: "I usually make a quick overview after my own consultation hours. 50\% of the patients I see here are out of place. They should have been sent to the outpatient clinic straight away." The most common reason for orthopedists to send patients from the center to the outpatient clinic is not because they suspect a serious problem that needs to be treated in the outpatient clinic, but because they feel they cannot say anything without x-ray or an MRI, or because protocols prescribe to order such diagnostic procedures. 42

M2 suggests a solution for this problem: "We need to look for the optimal routing in which the needed diagnostics are possible without having to refer the patient to the hospital." What M2 denotes "the optimal routing" is for patients to first go to the hospital to get an $\mathrm{x}$-ray done and then consult the specialist in the Primary Care Plus center about the problem. This has been discussed in previous meetings, where it raised objections, especially with general practitioners. M2: "General practitioners have a feeling - mind you, this is feeling not a fact, but a persistent feeling - that they are being controlled and judged." 43

Another means to improving the numbers is to keep the 'wrong' patients out of the centers. It is proposed that assistants at the outpatient-clinic will reconsider the referral letters of general practitioners, deciding what the best place is for specific patients: outpatient clinic or Primary Care Plus center. The head of the orthopedics department suggests figuring out on the basis of the numbers what kind of medical problems are suitable for Primary Care Plus. Head of the orthopedics department: "I would like to analyze these numbers a bit more in-depth. What can we learn from them, and what can we report back to our colleagues?"

Even without taking the deviant decision making of specialist Van Dongen into account, the orthopedists fail to perform as hypothesized. In the meeting, three other suggestions are made for improving the performance numbers. Having to

\footnotetext{
42 Often, this is seen as more of a problem for "invasive" specialisms, such as orthopedics, than for "reflective" specialisms, such as internal medicine or neurology, in which doctors are trained to carefully unravel the cause of a problem on the basis of symptoms, anamnesis and physical examination alone.

43 The director refers here to the discontent that general practitioners feel in the Netherlands and that also came up in many of my interviews with general practitioners, about the growing authority of health insurance companies.
} 
provide orthopedic care without imagery, orthopedist feel they cannot even start to think about whether the problem is serious or not and end up sending most of their patients to the hospital anyways. The organizers of Primary Care Plus, however, do not take this as an indication that Primary Care Plus does not work for the specialty of orthopedics. On the contrary, the natural response is to search for adaptations, in this case, a change in what is part of the core identity of Primary Care Plus: providing care without diagnostic procedures. The other suggested improvement is to get a better grip on what kinds of problems are right for Primary Care Plus, i.e. to change the input of the black box. Correcting the general practitioners' referral decisions is called "reverse-triage". The orthopedics department was the first to start doing this. Assistants who are trained for triage reconsider the referred patients and redirect them, if needed, to either the outpatient clinic or to the center. The head of department brings up the ambition of composing indication lists, extensively discussed in chapter three.

Analyzing the above observations in a performative idiom, we can draw three conclusions. First of all, if we take Primary Care Plus as a nudge, managing it is not merely a matter of implementing a stable design but an iterative process of constantly redesigning it. In order to arrive at the desired efficient outcomes, the conditions in which the nudge interferes are constantly tweaked. It is not just a change of location; more and more, it becomes a setting in which only specialists with specific personalities have exactly the right number of diagnostic procedures available to deal with only those patients whose problems are exactly the right ones for Primary Care Plus. Managing Primary Care Plus in practice becomes a balancing act of finding exactly the right conditions in which $75 \%$ of the patients can be helped sufficiently.

Secondly, as a nudge aimed at increasing efficiency, tweaking the conditions will naturally consist of taking out elements that do not or cannot confirm to the industrial rationality. Efficiency is not the result of changing one or two cues in the environment, but it comes into being through shedding elements that are irrational within the industrial world and adding elements that make the right kind of rational behavior possible.

If we return to the duality of design and action of nudging theory, we can draw the third conclusion. With this duality, a distinction is made between the nudger and the nudgee. While the first one is able to use its deliberative system to design a choice architecture built on deliberations, the second is assisted to make decision that match her true preferences. Analyzing the observations of a orthopedics meeting, however, showed that designing the nudge is most probably not finished before implementing it; it is a process that stretches over time and space. It remains 
a work in progress. This is not surprising, because viewed from the approach of this dissertation, the design is as much part of the realm of action as the nudge itself, making such fundamental differences unlikely. However, of concern is here that because a nudge is and will be a work in progress, because it is constantly shaped and reshaped, it is unclear whether 'desirable' outcomes are the result of a process of taking irrational actors out of the choice architecture or of making them act more rationally, i.e. according to their true preferences, through changing the environment.

\section{LEGITIMACY AND NUDGING}

In this last concluding section, I will return to the research question of this chapter, whether Primary Care Plus as nudge is a legitimate instrument for health care delivery. In a nutshell, the findings of the above sections have consequences for the ethical debate about the permissibility of nudging. This debate can be summarized in the following way: Nudging is criticized for bypassing the rational capacities of people, thus as a form of manipulation going against their autonomy. Proponents of nudging have two answers to this ethical problem. First, a nudge merely asssists people to choose what makes them better off according to themselves. Secondly a nudge is not more than a nudge; it is always possible to go against it. In the remainder of the concluding chapter, I will sketch three conditions under which the intentional change in the delivery of specialist care can be legitimate.

First of all, from the above findings, intentionally changing the choice architecture of specialist health care delivery is legitimate when it does not bypass the rational capacities of people, but it reinforces them. Changing the environment in which routine-like decisions are made potentially stimulates processes of reflection, which can help build new and improved skills and routines, and enforce a whole set of other moral skills, i.e. it can contribute to agent's moral scaffolding. Such a nudge does not have one predetermined right outcome but should be assessed with an open end, mainly aimed at generating creative processes. Note that when assessing a nudge by the momentary decisions it causes, i.e. by the outcomes it generates, these important and possibly productive processes of justification work are taken out of view.

Secondly, the empirical findings above render the first answer to the critique on nudging invalid. Rebuking the critique that nudging is a form of manipulation, proponents assure that such a change in the environment, intended to change the behavior of nudgees without them necessarily aware of it, can only be called a nudge if it assists nudgee's to make more rational choice, i.e. choices that agree with their true preferences. By closely tracking the justification work conducted in the consul- 
tation rooms, however, I brought into view that preferences emerge in the interactive and dynamic processes of justification work, instead of being a stable rational predisposition residing in the mind. More important than holding a nudge up to an unchanging standard of nudgee's actual preferences is to make sure that the change in the choice architecture enlarges or does not limit the space for a diversity of rich interactions, moral orders of worth, and preferences to emerge and dissappear.

The third important condition for Primary Care Plus to be a legitimate change in the choice architecture pertains to the second answer, by which proponents of nudging rebuke the critique that nudging is illegitimate manipulation. In this argumentation, a nudge is not detrimental to the autonomy of nudgees, because it is merely a nudge; there is always the option to not wash hands, to not take the stairs or to send the patient to the outpatient clinic in the hospital after all. This reasoning is only valid when taking the choice in which nudging intervenes as a momentary decision. Two of the above findings problematize this in two different ways. First of all, as the analysis of the ophthalmology shows, the decision to refer or not refer a patient to the outpatient clinic is not as clear-cut and isolated in time and space as the decisions nudgees make in the stories and examples by Thaler and Sunstein (2008). Because both specialist and patient do not enter the consultation room as a clean slate, there is a grey area, in which specialists and patients follow the direction of the nudge, but at the cost of something else they consider important, in this case, delivering optimal care, causing feelings of moral discomfort. Secondly, the example from the practice of designing and managing Primary Care Plus shows that the process of this nudge is an iterative one, in which the nudge itself and the conditions in which it interferes are constantly redesigned. As such, using nudging for policy or management purposes comes with the risk of forcing behaviour not by forcing a momentary decision but by slowly taking out the nudgees, in this case doctors, that refrain from behaving in a way deemed rational. In order to protect the changes in the choice architecture as a gentle push and not more than that, it is needed to protect the diversity of behaviours from a policy or management position.

Returning now to point of departure of this chapter, the third promise of Primary Care Plus, cost-efficiency through relocation, I will conclude this chapter with three notes. First of all, relocation can certainly be a fruitful innovation. Even when there are low patient numbers, or high referral rates, i.e. when the change of choice architecture does not have the intended effects, it might still have been a valuable intervention for the creative and reflective processes it has set in motion. Crucial, however, is to make these processes become visible, which is difficult when there are pre-defined criteria for success, i.e. when the process is merely assessed in regards to outcomes measures. A pioneer site should be a space for not only for controlled 
trials but also for experiments with open-end that create new and unexpected phenomena. Secondly, in order to make Primary Care Plus as a intervention intended to change behavior a success is when there is space for a diversity of moral orders of worth, understandings of what good care entails and interactive processes in which doctor and patient build skills for automatic and autonomous decision making, and for slowly and carefully shaping preferences. Third, legitimacy of Primary Care Plus as a modification of the choice architecture intended to change behavior, cannot be secured in the design phase alone. In order to achieve legitimacy, the autonomy of doctors, managers and patients involved in the delivery of Primary Care Plus needs to be protected in the process of delivering and managing it. This can be done by acknowledging autonomy as well as true preferences to be emerging instead of stable. Acknowledging that they are achieved in a process of justification work. If we want to assess such a management tool, we need to look at processes of design work, justification work and its implications. Diversity is key. 


\section{6 \\ CONNECTED AT THE JOINTS}

In the chapters of the dissertation, I explored the three signature promises of a prominent innovation in the delivery of health care that was set up as an alternative to organizing health care as a regulated market. The research that led to this dissertation was motivated by the observation that the health care market has become a huge point of contention in the public realm. The market was heralded in the early 2000s as a solution to the unceasing growth of the national health care system. Organizing health care as a market would render it lean, innovative, and efficient. Yet, while trust in the market is waning, the problems that led to introducing it are still as urgent as ever. In fact, health care researchers have been ringing the emergency bell: The ageing of the population, the increase of chronic diseases and multimorbidity, and new but expensive medical technologies are expected to result in unprecedented growth of health care expenditures. The latest national foresight study has projected that in 2040, health care expenditures per capita will have doubled in comparison to 2015 (RIVM, 2018).

This sense of urgency has proved to be a fertile soil for innovation. Blue Care and its intervention, Primary Care Plus, by which the consultations of medical specialists are relocated from the outpatient clinic in the hospital to a neighborhood care center, are just one of the many innovative health care delivery practices that started in the wake of the 2013 pioneer site policy of the Ministry of Health, Welfare, and Sports. The pioneer site policy, which became an official part of the ministry's policy, and their interventions were set up to achieve the so-called Triple Aim (Ruwaard et al., 2014): "care, health, and costs" (Berwick et al., 2008). The Triple and later Quadruple aim (Bodenheimer \& Sinsky, 2014; Van den Bogaart et al., 2019) were adopted in the national pioneer site policy to systematically monitor the interventions by measuring three (later four) different outcomes: population health, patient experience, costs, and provider satisfaction (added later).

In the introductory chapter, I showed that even though it was set up by critics of market care, when it comes to the ideas about how and why the pioneer site will improve health care, and who plays what kind of role, the ideology of Blue Care shares some important presuppositions with the market system. Both the market and Blue Care intervene in stakeholders' behavior by tapping into the norms that 
shape their behavior. Stakeholders, i.e. patients, medical professionals, insurers, and managers in Blue Care as well as market care are seen as isolated individuals who act in a material and social environment but are, at the same time, fundamentally separated from it. Innovating the delivery of health care, in this view, means implementing a system designed in the realm of knowledge, whether it is a market or an intervention that entails relocation and collaboration, which directs the more or less predictable behavior of stakeholders in the realm of action. Redirecting behavior in both the market system and in Blue Care is attempted through mitigating, enforcing or redirecting norms and preferences residing in the stakeholders' minds, presuming a separation between the mind, where knowledge and morality resides, and the body as an executor of the mind's intentions. Typical for such systems is the underlying understanding of agency. In this conception, agency is preserved for humans, who are possessors of preferences, intentions, and norms that shape how they behave while residing in their social material environment. The actions of such actors are more or less predictable and they can be directed through implementing a system.

Building on Pickering's (1995) sociology of science, I identified the above views of agency being set in the representational idiom. Pickering uses the difference between the representational idiom on the one hand and the performative on the other as a way of distinguishing two ways of writing and speaking about science. Phrased in the representational idiom, science is believed to be the activity of creating a body of knowledge that represents how the world is. In Blue Care's ideology and in market care, this idiom can be recognized in the dualism between the realm of knowledge, where the system is designed, and the realm of action, where, after implementation, it will direct the behavior of stakeholders. The abstract ideology of Blue Care assumed a more concrete form in its the actual interventions. Primary Care Plus, the relocation of specialist care from the outpatient clinics in the hospital to a primary care setting, was the largest intervention. Assessing the concrete interventions with the Triple Aim is natural within such set of assumptions. Measuring costs, patient experience and population health through, among other methodologies, distributing questionnaires is a way of creating a supposedly objective, reliable representation of the intervention-reality in a format that makes it easily comparable to other interventions in the delivery of health care in terms of costs, health and patient experience.

It is important to note here that I extended Pickering's representational idiom considerably for the analysis of the dissertation. While the representational idiom in Pickering's work pertains to science as a body of knowledge and an activity of making this body of knowledge increasingly representational of the world out there, in 
order to analyze Primary Care Plus, I broadened the idiom to also pertain to morality. The promises of Primary Care Plus are not only concerned with making medical knowledge increasingly representational of the human body, and the processes taking place in there, but also with improving the intervention-reality, i.e. with making it comply more and more with standards for and conceptions of, in this case, high quality, satisfactory care for reduced costs. While knowledge as representation is concerned with making an increasingly accurate representation of the world out there, morality as representation is an ideal or a standard of good care residing in the realm of knowledge that has a regulatory role in how health care is designed, implemented and assessed in the intervention-reality.

In his sociology of science, Pickering employs a performative idiom in his study of scientific practice. With such an idiom, there is no longer a fundamental gap between the world out there on the one hand and a body of knowledge representing the world on the other. Taking up the performative idiom means taking the world, including science itself, as constantly doing things, that is, as consisting of human and material agencies. Such a view allows for perceiving health care interventions, such as Primary Care Plus, and the methods used to assess them (Triple Aim) to be not outside of this world but to be part of it as well. They thus constanly and unpredictably make relations with and have a performative effect on other aspects of this world.

The Triple, later Quadruple Aim approach was reserved as a method for assessing interventions of the seven pioneer sites from the start of the pioneer site policy. My analysis of the marketing material, mission documents and interviews with initiators showed that besides functioning as an instrument that would objectively measure outcomes, these assessment methods also shaped the rationales about how the intervention would improve the delivered care. Resonating in the three core promises found in the document research and interviews with initiators, it shaped what professionals involved in the project promised and expected from it. While the vertical collaboration between general practitioners and medical specialists would induce learning and thus improve decision making and increase population health, the relocation would improve patient experiences and reduce unnecessary costs. As such, the intervention of Primary Care Plus and the internationally influential approach to health care innovation co-produced each other. The intervention came with a strong message about what it would improve. This paved the way for using the Triple Aim method, because the outcome measures of Triple Aim and the local 
promises were a near fit. ${ }^{44}$

Scholars such as De Wilde (2000) and Mesman (2011) warn that when absorbed in innovation, in this case, the complex and demanding process of developing, implementing and assessing new ways of delivering health care, the danger lurks of overlooking valuable but invisible routines, skills, (norms) and practices in the process. With the Triple Aim method for example, Primary Care Plus is assessed as an intervention. Keeping all other conditions stable, the effects of what is new in in the delivery of care, i.e. "the intervention", are measured. Yet, health care professionals and patients, absent and present diagnostic apparatuses, protocols and other material and human elements that make up the practices of organizing and delivering Primary Care Plus are involved in rich and dynamic processes that stay out of view. They are part of an already existing and continuous framework of skills, competences and convictions of what good care entails. In the course of the chapters, I have shown that such elements bring in a history of experiences, stories, associations, skills, and convictions, which shapes how professionals and patients deal with the new circumstances, tensions, problems, and opportunities.

Wilde (2000) and Mesman (2011) thus propose to exnovate. Instead of assessing an innovation or any other health care practice on its outcomes, it is important to look at what is done here and now. With such a focus, the dynamic practices as a continuation of already existing practices are made explicit in order to understand why they run well or how they can be improved. Adapted to this context, I have studied Primary Care Plus "exnovatively". This means, I have not focused on the effects of the break with "old care" but rather on how elements of "old care" thrive in the new environment of Primary Care Plus.

To be able to explicate the processes unfolding, i.e. in order to understand how Primary Care Plus was done there and then, I studied the management and delivery of Primary Care Plus as practices. Further substantiating the rather broad and diverse

\footnotetext{
44 When viewing and analyzing promises and practices with a performative idiom, the strict separation between intervention-reality in the realm of action and the neutral assessment method in the realm of knowledge vanishes. For the assessment method to function, the messy reality first needed to be rendered into an easily accessible, testable and researchable version of it. This is similar to Latour's (1999) notion of circulating reference. A stable reference between knowledge and the world out there does not miraculously appear when doing science or when running statistical procedures with the outcomes of questionnaires, but it is achieved by innumerable small steps, in which references are not merely made by creating representational knowledge but, importantly, by intervening and transforming the world that these references are meant to represent. In order to establish an acceptable reference between the care actually delivered at the center on the one hand and its documented patient-friendliness on the other, the university research team made employees of TIPP identify and enroll patients to the intervention and control groups - which proved a slow and tiresome process - they designed five questions for the questionnaires based on an internationally renowned index for researching patient satisfaction, they assembled forms, inserted them in envelopes and sent them out. In the period of ethnographic research, I observed the many small steps required to establish a convincing and scientifically valid reference between the consultations at the centers and the scientific data about how patients as a group experienced it. Implementing and assessing innovative health care interventions is thus a continuous process of making the world fit the representations created with the chosen assessment methods.
} 
family of practice approaches, I used Pickering's performative idiom mentioned above. As such, I understood the human and material aspects of the interactions of doctors and managers in management settings, and of patients and specialists in the setting of the consultation room, as emerging agencies. With such an approach, I went beyond the knowledge-action binary. I highlighted that assumptions about stakeholders' behavior and their moral convictions on the one hand and the diverse aspects of the intervention and its assessment methods on the other are part of one and the same world and thus mutually shape each other. Such a performative idiom creates space to study and dissect the practices of such shaping, instead of having to denounce them as spoilt by intervention. This counts for the scientific research practice of assessing Primary Care Plus scientifically but also for a management tool such as the indication list. While striving to be a neutral standard that prescribes whom to refer to the Primary Care Plus Center and who not, it is at least as interesting to track the actions required for embedding such an artefact as a useful tool in the ongoing process of managing the delivery of care.

Strauss' $(1985,1988)$ concept of articulation work proved to be particularly useful for understanding what was done when patients, managers and doctors delivered and managed Primary Care Plus as a practice of navigating human and material agencies. Articulation work is the mundane work required to make organizational processes run fluently - often rendered invisible afterwards. Explicating the mundane articulation work in two different settings showed how already existing moral and knowledge worlds merge in the management and delivery practices of innovating care. This yielded a rich source of knowledge about and understanding of the implicit skills, norms and routines of managing and delivering "old care" in a new setting. Note that assessment methods such as Triple Aim are precisely the kind of practices that render such work invisible. Having them in full view helps to better understand how to improve when it is not going well (anymore), how to set up similar interventions, and to understand which conditions and aspects are valuable and which are not. It helped, for example, to see that autonomy can emerge when immersing the patient in decision procedures, but also that changing the location of specialist care delivery can cause medical specialists to experience moral discomfort.

After three chapters of carefully tracking the moral and knowledge dimensions of articulation work, the main finding of this dissertation is that delivering and managing Primary Care Plus entails a variety of articulation work, in both moral and knowledge dimensions. In the consultation room as well as in the meeting room, patients, doctors and managers constantly make efforts to resolve, connect and accommodate knowledge and moral differences in order to achieve durable deci- 
sions. The successes of and opportunities created by the intervention identified in this dissertation should not be taken as merely an effect of the intervention's design but of its interplay with the diverse and productive knowledge and justification work enacted by the professionals and patients involved in Primary Care Plus. Assessing a new way of delivering care should not only take into account the effects of the new, but also the already existing soil on which the new is put to work. In the remainder of the chapter, I will further explicate what we can learn from this finding for theory and practice.

\section{ARTICULATION WORK}

Analyzing the articulation work conducted to make Primary Care Plus run, I found that the notion as introduced by Strauss $(1985,1988)$ and further elaborated by other sociologists (Bardram \& Bossen, 2005; Postma et al., 2015) needs to be broadened. Strauss and others who followed his tracks, have mainly focused on its temporal and spatial character. In the third chapter of the dissertation, I found that articulation work has a knowledge dimension and, in the fourth chapter, a moral dimension as well.

Primary Care Plus is organized and delivered by a group of health care professionals from different disciplines, with a variety of experiences, skills, and convictions about what good care entails. Likewise, the medical specialists delivering Primary Care Plus in the consultation room encounter a variety of patients with different backgrounds, convictions, experiences and stories. In the settings of my observations, the users' council meetings and the consultation rooms, patients and professionals constantly navigate existing and emerging human and material agencies. On the spot, they come across and are required to deal with reactions and preferences of others, but also by invoked procedures and protocols and absent technologies. In this constant navigation of agencies, moral worlds clash or merge; joints are created between contrasting realities. But most importantly, neither treatment nor organizational decisions are taken; they are achieved.

In order to achieve durable decisions in meetings and in the consultation room, I found professionals and patients conduct a diversity of knowledge and justification work. Whether it is about organizational problems encountered on the work floor or about the course of the organization for the future, solution or problem realities clashed in the meeting room. In order to achieve organizational decisions, the tensions between these realities needed to be dealt with by accommodating, bridging or solving the differences. In the consultation room, where I tracked how patients and specialists navigated material and human agencies by resistance and accommodation, I found patient and doctor achieving treatment decision through 
aligning moral worlds, merely aligning outcomes, or agreeing on procedures. Most of the time, the doctors and patients I observed were aware that treatment decisions need to be durable. A durable decision is not merely a correct one but also one that is achieved through sufficiently dealing with tensions between moral worlds. This is done through justification work, which shapes and produces notions of good care.

The acts of knowledge and justification work that I found in the observations described in the second, third and fourth chapters was diverse. Professionals involved in the discussions disentangled and explicated examples of the problem at hand, appropriated part of the problem realities of others, reconfigured the fit between problem, solution and justification realties, entangled future work processes through imagination, shifted focus from process to content, or relocated the decision making to another setting. I also found diverse acts of justification work put to work to achieve durable treatment decisions. To name a few: Doctors used analogies, conducted different kinds of examinations, listened closely to their patients, built trust through jokes or dialect, turned the computer screen, or showed the uncertainties involved in making diagnoses and treatment choices. Such acts are not just a matter of treating the patient well but are crucial for shaping how the patient accepts or resists the trajectory ahead, whether it leads home or further into the health care system.

By explicating acts of knowledge and justification work, it became clear that the two kinds of articulation work show some striking resemblances. First of all, often, such work is not aimed at fully aligning or integrating knowledge or moral worlds but at sufficiently attuning them. Processes of managing and delivering health care often proceeded after merely joints were found, through which aspects of the different moral and knowledge worlds could flow from the one world to the other. Different kinds of elements functioned as joints in the observed processes. In the discussion about defective referrals of general practitioners for example, no full agreement about the problem realities was found when the organizational processes started flowing again. The discussion started off with the present rheumatologist recounting a dramatic story of a child with RA being wrongly referred to the Primary Care Plus center. In the course of the unfolding knowledge work, RA, a medical problem that evidently does not belong at the center became exemplary for all problematic referral behavior. As such, it was used by each of the professionals taking part in this discussion - for example when projecting what would happen with such a faulty referral in case proposed solutions had been implemented - even though their problem realities clashed. As such, it became what I denoted a "joint of agreement". In the consultation room, some of the justification work entailed finding joints instead of fully aligning orders or worth. At one of the rheumatology 
consultations, the protocol that the specialists followed and explicated was the joint that enabled sufficient attunement between patient and doctor. For both the patient for whom the civic order of worth is appropriate and the rheumatologist who justifies her decisions within a combined industrial-domestic order of worth, a protocol, is a worthy object, even though it has a different meaning within the one and the other order of worth. Explicating the protocol behind the treatment decision that she proposed, enabled doctor and patient to achieve a durable treatment decision without needing to align or even compromise their orders of worth.

Secondly, processes of knowledge and of justification work are not necessarily linear. In the studied discussion at the user's council meeting, the problem and solution reality were first broadened and then narrowed down again. In this process, the meaning of certain material aspects kept changing. First, the indication list was proposed as a tool to provide the lacking information to general practitioners. Later in the discussion, it became a process in which general practitioner and specialist working together would create mutual understanding of each other's perspectives. After, it became algorithmic-like device, helping assistants to make decisions without having the needed knowledge or skills. Last of all, meanings were dissipated, rendering the list into nothing more than a way of providing the missing information. This process was thus not linear but constructive and destructive. Knowledge and meanings were gained and subsequently lost. In the consultation room I noted similar processes: an eye operation that could open up a clogged up tear duct transformed from a simple intervention into an invasive surgery, bearing comparison to "chopping, cutting and breaking a sewage pipe". Along the way, the tearing eye turned from a serious nuisance into a "minor inconvenience". In other versions of the same process of gain and loss, preferences emerged and dissipated, autonomy was gained and lost, which values should be prioritized shifted from one to the other.

The third general characteristic of the two kinds of articulation work is that such processes are sequential; they go beyond the timespan and setting of a single meeting or consultation. The indication list as a solution to the problem of faulty referral behavior, for example, only became an evident solution for the problem after it had been embedded in sequences of knowledge work conducted at different times and in different places. In order to make the list into an appropriate or even self-evident solution, it was not enough to compose and subsequently present it. In the chapter three, I described such work in two different users' council meetings: one, in which it was first suggested as a solution and a second one, in which an actual list composed by a team consisting of a manager, a general practitioner and a medical specialist was presented to other medical professionals. During the ethnographic 
research, I observed the indication lists flowing from one to another setting, once and again being presented, explicated, justified and opened up for improvements. The work required to make the indication list as a solution fit the problem at hand is ongoing and flows from one setting to the other. The list's workability is achieved not only through constantly tweaking the solution itself, but also the social and material context in which it is put to work.

Similarly, processes of justification work are not just instances of momentary tinkering with moral worlds, but they start before and extend beyond the time spent in the consultation room. I described how such work often entails creating a somewhat coherent moral framework in which past and future events are connected, and in which the treatment decisions need to be embedded in order to become understood as the right decision for this specific patient. The frameworks that are built or invoked in the processes of justification work are important, because they allow for the development of skills and routines that are helpful in for example achieving trustful relations with patients who have lost trust in their doctors, their bodies and the health care system.

Last but not least, knowledge and justification work follow a certain logic and as such are a reasoning processes. Analyzing the observations of the users' council meetings, I identified three different epistemic logics that I denoted "logics of integration, connection and distribution”. These logics are akin to schools of epistemology, but in contrast, they are not abstract reasoning processes unfolding in the mind, but they are processes taking shape in interaction with the social and material environment. The professionals conducting knowledge work do not stick to one logic. They follow the one logic in one setting and another in another, depending on which one they deem appropriate. I found participants, in the course of the knowledge work conducted in one and the same meeting, shift from one to the other in order to achieve sufficient attunement between different problem, solution and justification realities. In the chapters about justification work, the orders of worth collected by Boltanski and Thévenot proved useful. What justification work patient and doctor conducted or how they perceived the justification work of others depended on which common good doctor and patient considered of highest worth. Sometimes it was proximity and trust, other times efficiency or autonomy.

The above stands in stark contrasts with the approach used to assess Primary Care Plus and the other interventions in the pioneer site policy. Measuring instruments such as the Triple or Quadruple Aim methods foreground health outcomes, patient (and provider) satisfaction and cost reduction. Such a way of assessing a practice, I argued in the dissertation, departs from a set of dualisms that separates knowledge from the realm of actions it represents, the body as an executor of the 
mind's intentions and the agent as fundamentally separated from the structures in which she acts. Also, it assumes that it is possible to assess the intervention without shaping it. Taking up the performative idiom, instead, allows for viewing Primary Care Plus exnovatively and, thus, tracking the implicit processes of achieving durable decisions by navigating emerging human, social and material agencies. In the analysis conducted in the chapter, I took the observations done in the ethnographic research and the reflections on them with specialists, managers and patients as part of the studied practice. Whether it was the setting of the users' council meeting or of the consultation room, in order to achieve durable decisions, the emerging moral and knowledge worlds of involved professionals and patients and of (the absence of) technologies needed to become sufficiently attuned. The knowledge and justification work processes conducted in these processes were often non-linear and sequential. They entailed establishing joints rather than reaching full integration, and they followed certain epistemic logics or orders of worth.

\section{THE EMERGING AGENCIES OF PRIMARY CARE PLUS AND BEYOND}

Going back to the three promises of Primary Care Plus and the trends in the delivery of health care that they resonate with, the practical implications of the findings of the dissertation become clear. The first promise of Primary Care Plus that I identified is that the vertical collaboration, i.e. the collaboration between general practitioner and medical specialists, will yield a learning process. Primary Care Plus' promise of learning through collaboration, I argued in the second chapter, fits the current trend in health care to use care integration, i.e. the collaboration of professionals from different care sectors as a strategy to "enhance quality of care and quality of life, consumer satisfaction and system efficiency" (Kodner \& Spreeuwenberg, 2002, p. 3). To be more specific, the promise of learning through vertical collaboration fits the model in which medical knowledge of different health care professionals is integrated and thus improved through collaboration.

In the third chapter, I showed how Integrated Care does not unproblematically flow from designing a model in which professionals from different disciplines work together. By tracking integrated-care-in-action, thus, the work required to make interdisciplinary collaboration run fluently, I learned that it is not so much full alignment (integration) that is required. Even after the diverse and extensive knowledge work conducted by the professionals participating in making organizational decisions in the Primary Care Plus meetings, there was still a rather high level of fragmentation between knowledge worlds. In literature, interdisciplinary collaboration is often defined as collaboration across disciplinary boundaries (Kodner \& Spreeuwenberg, 2002). Yet, the above described findings of the dissertation renders the 
metaphor of boundaries and borders in speaking and writing about such collaboration problematic. When managing, conceptualizing, or understanding interdisciplinary collaboration, it might be more productive to think in terms of sequences and joints than of areas that are separated by boundaries that need to be crossed. These findings also endorse Szerszynski and Galaragga's (2013, p. 2817) critique on what they call the "integrative imaginary", by which we "mistakenly assume that disciplines can be combined in a straightforward way to reveal different aspects of the same underlying world". In line with their argumentation, I would argue for a more reflexive imaginary for interdisciplinary collaboration in the delivery of health care, i.e. in conceptualizations of Integrated Care,one that makes space for heterogeneity and multiplicity of knowledge worlds of different disciplines involved in collaborating.

In literature about Integrated Care, a tendency to strive for homogeneity of knowledge can be discerned. With the findings of chapter three, I would argue the opposite. Full integration of knowledge, a homogeneous body of knowledge within an organization, is not needed and might even cause problems in the practice of managing and delivering health care. Full integration comes with reduction of knowledge, inevitable when making different and incompatible knowledges fit one body. Such reduction often comes with a hierarchy. It depicts a medical specialist to have in-depth knowledge about one specific specialty, and general practitioners have broad but superficial knowledge about diverse specialties. When taking these knowledges as compatible, as able to be integrated, a specialist is often considered more knowledgeable about one topic than a patient. Instead of striving for homogeneity, I argue here that it is the heterogeneity that should be protected, counterintuitively, even in practices of Integrated Care.

In the instances of knowledge work that I have shown, full integration was hardly ever needed for organizational processes to run fluently. Often, professionals proved to be able to agree on a course of action without resolving differences, i.e. while maintaining the internal diversity. Connecting through joints, as the anatomical metaphor suggests, allows for flexibility. Internal knowledge differences allow for a collective to be more attentive to signals and frictions, for upcoming problems and opportunities. Furthermore, a heterogenous collective is more productive in coming up with creative solutions for unexpected obstacles on the road. For the interdisciplinary organization of Primary Care Plus to flourish, space for a diversity of knowledge practices and for the work required to create joints between them is crucial, not getting the different professionals to integrate their diverse and often conflicting bodies of knowledge.

In practice, protecting heterogeneity means being mindful when using reductive management devices that discipline doctors, patients, and professionals to act in a 
uniform way, such as basing management decisions on outcome measures alone, adopting uniform schedules, or formats for the indiciation lists. Another point of attention would be to keep the tendency to define problems top-down in check. For example, in the third chapter, I described how in the course of a discussion in the users' council meeting, the referral problem became the problem of general practitioners not referring in a way that would be right from the medical specialist's point of view. This tendency became especially noticeable when the scientific research was mentioned that based its analysis merely on the final diagnoses made by the medical specialists during specialist consultations. In contrast, the plan to make teams of medical specialists and general practitioners compose indication lists together can have the opposite beneficial effect on the internal knowledge heterogeneity. If such a team succeeds in incorporating insights from both positions, this will create space for heterogeneity in the organizational processes of Primary Care Plus and in how the actions of involved doctors are evaluated.

This brings us to the learning aspect of the first promise of Primary Care Plus. By reconceptualizing the notion of Integrated Care and particularly the knowledge concept presumed in its promises and expectations, the learning effect seems to have become irrelevant. Knowledge in the analyses of chapter three is not something like a commodity that can be unpromblematically transferred from the one to the other, neither something that resides in the mind and can be obtained by exchanging knoweldge with people who have other knowledge in their mind (mentalist view); it is a practice in which sufficiently attuning the differences is crucial for making organizational processes run. However, as became clear in the fourth chapter, even if we take Primary Care Plus as a practice of delivering and managing specialist care, learning processes are and should be central to how the organization is managed and assessed.

In the fourth chapter, I presented how learning processes could help develop Primary Care Plus as a specific form of specialist care. Space for experimentation for providers of Primary Care Plus, I argued, potentially yields fruitful new views, skills and solutions for broader problems in the Dutch health care system. For example, when analyzing the consultation of internal medicine, it became clear that the specialist had developed an intricate routine of dealing with patients with what she referred to as "trust issues". Such patients, in her view, often have a problem finding the care they need and will go from specialist to specialist. This is particularly interesting for the intervention of Primary Care Plus, because it seamlessly fits one of its most important goals to help keep the Dutch health care system accessible in the future. In the assessment methods of Primary Care Plus, the emphasis has been on making the care delivery efficient without being detrimental to the quality 
and accessibility of care. A noted but underexplored possible contribution of the intervention is to develop Primary Care Plus as a new and promising form of care in how it is done in the consultation room. The example of the specialist of internal medicine could function as an exemplar and thus a starting point of developing the conditions in which new and unexpected routines will likely come up. Importantly, developing such a routine, I emphasized, had not been an abstract process unfolding in the mind of the specialist (mentalist view) but a practice of action and reflection. In practice, such processes could be stimulated and noted by organizing, for example, regular training sessions in which specialists view how they themselves and others deliver Primary Care Plus to reflect on and learn from. ${ }^{45}$ However, typically such processes will not have immediate visible positive effects on the cost-efficiency. Allowing space for experimentation and making such experimentation fruitful requires patience and a longer vision for the future.

The second promise of Primary Care Plus is that by relocating specialist consultations from outpatient clinic in the hospital to two neighborhood centers, specialist care would become more accessible. In marketing material, interviews and mission documents, this was emphasized both in a literal (the centers are close to where patients live) and in a transitive sense of the word (friendly, non-hierarchical social interactions between patients and doctors). With the Triple Aim approach, the effects of such accessibility was measured as part of improved patient experience.

Focusing on the patient experiences as one of the three important aims of the care intervention, I argued in the second chapter, fits the current emergence of patient-centered care. The many review and research articles that have been published about patient-centered care can be read as a quest to find the essence of patient-centeredness or the correct yardstick that would enable to compare different practices in the same terms. As such, this quest is phrased in the representational idiom, because it is the activity of finding the right methods and definition to enable creating assessments of practices that reflect or correspond to how patient-centered the intervention actually is. I argued that a representational notion of morality was assumed, because the intervention is seen as a way to make the intervention-reality comply increasingly to predefined standards of patient-centeredness or preset categories that make comparison possible.

On the basis of the ethnographic research, I concluded in the fourth chapter that while most of such conceptions of patient-centeredness understand it as a measurable quality of delivered care, it is more informative to understand it as a process, to be more specific, the creative process of justification work between doctor and patient. Such justification work is often non-linear, sometimes produc-

45 A suitable method for this could be for example video reflexive ethnography (Iedema et al., 2019). 
ing conflicting meanings of what patient-centeredness entails in one and the same consultation. More important than finding what it actually is in the world or how to objectively measure it is to reflect on such creative processes in collaboration with professionals themselves. What do they do to achieve durable decisions? Knowing what goes well, how can they use it more structurally, thus improving how they deliver care patient-centeredly? More important than distributing questionnaires that enquire into a predefined checklist of patient-centered experiences is also, in this case, to train Primary Care Plus specialists to become aware of how they do patient-centered care. How do they feel out, deal with and make space for the diversity of moral convictions about what good care entails. Such a training would not entail practicing how to follow instructions, models of good patient-doctor interactions, or learning how to apply a protocol in diverse situations; it would depart from the professionals existing skills and competences in dealing with patients in the consultation room, reflection on what was done well there and then, comparing with others, and, from reflecting on it, find points of improvement and lessons for others.

The third promise I identified is that offering specialist consultations in a primary care setting would assist patient and specialist to take fewer unnecessary treatment decisions. As such, Primary Care Plus was promised to have potential for reducing health care costs. This potential was explained by contrasting the lean Primary Care Plus centers with the bureaucratic giants with huge overhead costs that hospitals have become. More importantly, in a primary care setting, rather than aiming to go to the bottom of the problem, specialist and patient would tend to think more like general practitioners: not doing more than feeling out whether there is a serious problem that needs more advanced specialist care or not.

The way in which Primary Care Plus and its mechanism of cost reduction are conceptualized in the third promise resonates with the policy tool of nudging. A nudge is a subtle change in the choice architecture, assisting people in taking the right choice. When nudging was presented as a new and promising policy tool, an alternative to banning options or directing people through informing them about benefits of making the right choice or the disadvantages of making the wrong choice. Nudging would help people to get on the right track without the risky and tiresome process of convincing them about what is right and wrong. After its introduction, an ethical debate took off, focusing on the question whether or not nudging, as a tool that bypasses the rational capacities of people, is detrimental to the autonomy of nudgee or not. Proponents of nudging have formulated two general answers to rebuke the critique. First, a nudge is merely a nudge; it is always possible to go against it. Secondly, a nudge merely nudges a nudgee in the direction that would make her better off according to her own standards. 
Again, the promises of Primary Care Plus and the argumentation of proponents in the debate about the legitimacy of nudging is stuck in a representational idiom that does not do justice to the rich processes of justification work that unfold in the consultation rooms of Primary Care Plus as a nudge. I argued that tracking the work conducted to achieve durable decisions showed that two crucial assumptions of proponents are untenable. First of all, behind the actions of an agent there is not a set of "actual preferences" that actions are or are not an expression of. Preferences are not given beforehand; they come into being in the rich process of justification work that unfolds between the material and human agencies in the consultation room. Secondly, autonomy is not something that an agent possesses and that needs to be protected, it is the interactive process of justification work, in which the autonomy of doctor and patient is shaped. On the basis of the found process of achieving durable treatment decisions, I argued that the process of designing a nudge as well as the decision making in consultations is a process in which autonomy, preferences and the design of the nudge itself are subject to change. A more workable criterium is that for such an intentional modification of the choice architecture to be legitimate, it should not bypass processes of justification work but either generate them or leave space for a diversity of moral convictions about what good care entails. This could be done by refraining from placing "the right" specialists at the center and keeping the unsuitable ones out, but involving also the ones that perform differently in the above mentioned reflection sessions. Taking specialists out of their usual work environment, as said, can be particularly fruitful, provided that they are seen as sources of insight into what Primary Care Plus could entail, instead of taking them as cogs in the machine that should be taken out if not performing as expected. The legitimacy of Primary Care Plus, as the relocation of specialist care with the intention of directing behavior, depends not only on how much autonomy patient and specialist have in the moment of taking a treatment decision (are they allowed to refer to the hospital or not) but on how the intervention is assessed and managed. Changes in the choice environment, in theory, could bypass rational capacities, but in the studied practices, I mainly saw the rational capacities of nudgees being turned on. It is thus crucial to refrain from rendering such valuable and unexpected processes and opportunities invisible while focusing on referral rates as the predefined desirable outcome of the nudge. The outcome of such changes are unpredictable, because they intervene in a playing field in which meanings, preferences and conditions are subject to change. This makes it important to monitor the implementation of such a relocation because of the unexpected problems. However, monitoring should be done with an open-ended outcome. Otherwise, as became clear in the fifth chapter, implementing the change becomes an iterative process of slowly filter- 
ing out deviant elements, which would be detrimental to the claim of allowing the nudgee to set her own course.

In each of these practical implications, in one way or the other, the importance of creating space for, explicating and becoming skilled in feeling out and conducting knowledge and justification work is of central concern. The findings of the dissertation have implications for how we assess health care innovations. One of the central messages is that we should use open-ended methods more often that not only focus on the outcomes but also on the processes unfolding. With other words, instead of merely measuring outcomes, we should exnovate. Another related one pertains to the knowledge and justification work I tracked in the dissertation. Such work is thus not only important for the sake of making processes of health care delivery and management run fluently but also to enhance reflection and learning processes. A good doctor does not only make the correct diagnosis and selects the right treatment; she is also skilled in developing new routines of justification work to deal with constantly emerging and reemerging problems of patients dealing with a diversity of problems that are not merely social, trust-related or medical, but all three at once. Similarly, professionals need to be skilled in knowledge work in order to contribute to well-running organizational processes. Here as well, a good professional is not preoccupied with aligning the existing problem, justification and solution realities but in shifting from one knowledge world to the other, finding a common course of action, while giving space for heterogeneity. Such skills cannot be developed by reading books but by practicing again and again, viewing and explicating her own and others' processes of justification work that shape situated understandings of good care (management), i.e. it involves exnovation.

\section{EMPIRICAL ETHICS}

In the research project that led to this dissertation, I did not only study a group of professionals and patients working hard to make interdisciplinary collaboration bear fruit; I also sought to combine different disciplines myself. To be more specific, in the research I conducted, I used and borrowed ideas, concepts, methodologies and theories from Ethics and Moral Philosophy, Science and Technology Studies, Health Sciences and Behavioral Economics.

Needless to say, I never intended to or reached full integration between these different disciplinary fields. What I did do is attempt to arrange elements of each in such a way that they would constructively inform a convincing and insightful argumentation. For example, literature in Behavioral Economics and Health Sciences helped me to identify the assumed rationales and conceptions implied in how Primary Care Plus is marketed and managed. This was the point of departure for 
presenting the ethnographic data and argumentation.

At the heart of the dissertation, however, lies the strained relation between Ethics and Moral Philosophy on the one hand and the more empirically-oriented discipline of Science and Technology Studies on the other. It is common in Ethics and Moral Philosophy to distinguish between ethics and morality. Morality encompasses the practical routines and norms that people have developed to maintain well-functioning relations with each other and by which people attempt to achieve the good life. In this view of morality, such norms and routines have become so self-evident that they often stay implicit. Morality can be studied empirically. For example, sociology of morality would study the broadly accepted practical routines and norms structuring the social behavior of people in society, like moral psychology is focused on processes of moral reasoning. Contrary to morality, which is usually hidden, ethics is the explicit discussion that emerges whenever a tension comes up between moral norms and routines of people among each other (Swierstra, 2009). The academic study of ethics is concerned with how to make the morally right decision when the wishes, interests and/or rights of others are of concern. Applied ethics, then, is the application of these systematic ways of thinking on actual cases.

In the above simplified overview of studies of ethics and morality, there is a clear distinction between empirical and philosophical studies. Note, however, that this distinction is based on the presumed duality between morality, the hidden and self-evident, i.e. immanent, norms and routines that are effective in a society and that structure individual behavior of people on the one hand, and ethics as the (un) systematic discussion about morality that erupts when people disagree about moral norms and routines on the other.

In the past decades, different researchers have carved out a position for themselves in between the social study of morality and the normative study of ethics, making sense of the moral/ethical world around them without distinguishing categorically between morality and ethics. Highly schematized, in this research field in between disciplines, it is possible to distinguish two different emphases in merging ethics and morality. First of all, researchers such as Mol (2008) and Pols $(2015 ; 2018)$ focus on how daily care activities are shaped by immanent rationales or values of good care. They study ethics as if it were morality, as embedded routines, without presupposing such morality to be self-evident or widely accepted. In their ethnographic descriptions and the analyses thereof, logics and values shaping care practices sometimes align, but often, there is friction or they outright clash. The work of Sayer (2011) represents another position in between empirical and normative studies. The problem of the social sciences, according to him, is that 
it ignores that we are beings whose relation to the world is one of concern. Concepts such as 'preferences, interests, values, (...) conventions etc. (...) are useful for external description, but can easily allow us to miss people's first person evaluative relation to the world and the force of their relations. (2011, p. 2)

Not the immanent rationales and values are of primary concern for Sayer to conceptualize his middle position but the importance of the first-person perspective that people have on the world around them, with which they are deeply and morally involved. Foregrounding that people are "sentient, evaluative beings" (2011, p. 1) resonates with Boltanski and Thévenot' (2006) analytical framework that, as explained in the fourth chapter, starts from the observation that the common and daily justification work that people conduct has been neglected in the social sciences.

In this dissertation, I have sought to combine these two positions in between disciplinary fields in the research and analysis that resulted in this dissertation, comparable to Swierstra's (2002) call for an approach that combines the emphasis on immanence on the one hand with the importance of the first-person perspective on the other. Swierstra (2002, p. 37):

\begin{abstract}
Our existence unfolds in a playing field that can only be surveyed from viewpoints within the field. That is immanence. (...) the normative philosophy that I call for tries to anticipate on this by emphasizing that we can never know whether norms, values, vocabularies can be relocated, but that it does not hurt to examine the option, (...), by understanding normative solutions as temporary and experimental, open to review, by not taking dilemmas, conflicts, grey areas, and blind spots out of view but to showcase them broadly, by recognizing everybody performs reality - philosophy in the wild, (...) by connecting prudence to morality.
\end{abstract}

Adapted to the context of the study, I have strived to make the grey area of immanent, clashing and aligning values, routines and convictions of good care delivery and management visible, reflecting with professionals and patients involved in achieving durable treatment and organizational decision on meetings and consultations. That most of the moral routines, norms and convictions are implicit and tacit does not mean that they are self-evident and widely accepted. By viewing Primary Care Plus exnovatively, tacit routines, norms and first-person perspectives of these different actors became explicit and, thus, also the hidden tension and differences between them. Important in the analyses of the research were the actors' convictions of good care (delivery and management), built through experiences 
and reflections that give shape to the ways in which they are concerned with the world around them and interact with it. In the course of the dissertation, I brought into view that even though much of the justification work was hidden, the moral worlds were not always aligned, neither did they need to become fully attuned in order to make organizational and delivery processes run. Tracking, dissecting and analyzing the justification and knowledge work that people conduct when achieving durable decisions as shown enables informed ethical assessment while doing justice to rich and dynamic practices. 


\section{REFERENCES}

Aggarwal, A., Davies, J., \& Sullivan, R. (2014). "Nudge" in the clinical consultation. An acceptable form of medical paternalism? BMC Medical Ethics, 15(1). doi.org/10.1186/1472-6939-15-31

Allen, D. (2014). The invisible work of nurses. Hospitals, organisation and healthcare. Routledge.

doi.org/10.4324/9781315857794

Apesoa-Varano, E. (2013). Interprofessional conflict and repair. A study of boundary work in the hospital. Sociological Perspectives, 56(3), 327-349. doi.org/10.1525/sop.2013.56.3.327

Balint, E. (1969). The possibilities of patient-centered medicine. The Journal of the Royal College of General Practitioners, 17, 269-276.

Bardram, J., \& Bossen, C. (2005). Mobility work. The spatial dimension of collaboration at a hospital. Computer Supported Cooperative Work: CSCW: An International Journal, 14(2), 131-160.

doi.org/10.1007/s10606-005-0989-y

Barrett, M., Oborn, E., Orlikowski, W., \& Yates, J. (2012). Reconfiguring boundary relations. Robotic innovations in pharmacy work. Organization Science, 23(5), 1448-1466.

doi.org/10.1287/orsc. 1100.0639

Bechky, B. (2003). Sharing meaning across occupational communities. The transformation of understanding on a production floor. Organization Science, 14(3), 312-330.

doi.org/10.1287/orsc.14.3.312.15162

Bechky, B. (2006). Gaffers, gofers, and grips. Role-based coordination in temporary organizations. Organization Science, 17(1), 3-21.

doi.org/10.1287/orsc.1050.0149

Berendsen, A., Benneker, W., Meyboom-de Jong, B., Klazinga, N., \& Schuling, J. (2007). Motives and preferences of general practitioners for new collaboration models with medical specialists. A qualitative study. BMC Health Service Research, 7, 4 .

doi.org/10.1186/1472-6963-7-4

Bertschy, S., Pannek, J., \& Meyer, T. (2016). Delivering care under uncertainty. Swiss providers' experiences in caring for women with spinal cord injury during pregnancy and childbirth - an expert interview study. BMC Pregnancy Childbirth, 16(1), 181 . 
doi.org/10.1186/s12884-016-0976-y

Berwick, D., Nolan, T., \& Whittington, J. (2008). Triple Aim. Care, Health and Cost. Health Affairs, 27(3), 759-769.

Birnbach, D., King, D., Vlaev, I., Rosen, L., \& Harvey, P. (2013). Impact of environmental olfactory cues on hand hygiene behaviour in a simulated hospital environment. A randomized study. Journal of Hospital Infection, 85(1), 79-81.

doi.org/10.1016/j.jhin.2013.06.008

Blumenthal-Barby, J. S., \& Burroughs, H. (2012). Seeking better health care outcomes. The ethics of using the "nudge". American Journal of Bioethics, 12(2), 1-10. doi.org/10.1080/15265161.2011.634481

Bodenheimer, T., \& Sinsky, C. (2014). From triple to quadruple aim. Care of the patient. Annals of Family Medicine, 12(6), 573-576.

doi.org/10.1370/afm.1713

Boltanski, L, \& Chiapello, E. (2007). The new spirit of capitalism. Verso.

Boltanski, Luc, \& Thévenot, L. (2006). On justification. Economies of worth. Princeton University Press.

Bontemps-Hommen, C., Baart, A., \& Vosman, F. (2018). Practical wisdom in complex medical practices. A critical proposal. Medicine, Health Care and Philosophy, $22,95-105$.

doi.org/10.1007/s11019-018-9846-x

Bovens, L. (2008). The Ethics of Nudge. In T. Grune-Yanoff \& S. Hansson (Eds.), Preference change. Approaches from philosophy, economics and psychology, (pp. 207-220). Springer.

Bresnen, M., \& Harty, C. (2010). Editorial. Objects, knowledge sharing and knowledge transformation in projects. Construction Management and Economics, 28(6), 549-555.

Bresnen, M. (2009). Living the dream? Understanding partnering as emergent practice. Construction Management and Economics, 27(10), 923-933.

doi.org/10.1080/01446190902974145

Bresnen, M. (2010). Keeping it real? Constituting partnering through boundary objects. Construction Management and Economics, 28 (6), 615-628.

doi.org/10.1080/01446191003587711

Bronchetti, E., Huffman, D., \& Magenheim, E. (2013). When a nudge isn't enough. Defaults and saving among low - income tax filers. National Tax Journal, September, 609-634.

Bruijnzeels, M., \& Kommer, K. (2013). "Durven delen, dat rendeert.” De Eerste Lijns. Platform voor Strategie en Innovatie, 6-9.

Carlile, P. (2002). A pragmatic view of knowledge and boundaries. Organization 
Science, 13(4), 442-455.

Castro, E., Van Regenmortel, T., Vanhaecht, K., Sermeus, W., \& Van Hecke, A. (2016). Patient empowerment, patient participation and patient-centeredness in hospital care. A concept analysis based on a literature review. Patient Education and Counseling, 99(12), 1923-1939.

doi.org/10.1016/j.pec.2016.07.026

Cleary, P., \& Edgman-Levitan, S. (1997). Health care quality. Incorporating consumer perspectives. JAMA, 278(19), 1608-1612.

Coeckelbergh, M., \& Mesman, J. (2007). With hope and imagination. Imaginative moral decision-making in neonatal intensive care units. Ethical Theory and Moral Practice, 10(1), 3-21

doi.org/10.1007/s10677-006-9046-2

Cohn, S. (2014). From health behaviours to health practices. An introduction. Sociology of Health and Illness, 36(2), 157-162.

doi.org/10.1111/1467-9566.12140

Cooke, M. (2013). A more ambitious agenda for medical education research. Journal of Graduate Medical Education, 5(2), 201-202.

doi.org/10.4300/jgme-05-03-42

Courtney, M., Spivey, C., \& Daniel, K. (2014). Helping patients make better decisions. How to apply behavioral economics in clinical practice. Patient Preference and Adherence, 8, 1503-1512.

doi.org/10.2147/PPA.S71224

Darby, C., Crofton, C., \& Clancy, C. M. (2006). Consumer assessment of health providers and systems $\left(\mathrm{CAHPS}^{\circledR}\right)$. Evolving to meet stakeholders needs. American Journal of Medical Quality, 21(2), 144-147.

doi.org/10.1177/1062860606286263

De Boer, D., Delnoij, D., \& Rademakers, J. (2010). Do patient experiences on priority aspects of health care predict their global rating of quality of care? A study in five patient groups. Health Expectations, 13(3), 285-297.

doi.org/10.1111/j.1369-7625.2010.00591.x

De Recht, Lieve \& Co. (1992). Camembert, rechtvaardigen en geweldloze liefde. Het politiek-filosofisch-economisch-sociologisch-antropologisch raster van Luc Boltanski en Laurent Thévenot. Krisis, (47), 5-22.

De Wilde, R. (2000). Innovating innovation. A contribution to the philosophy of the future. Policy Agendas for Sustainable Technological Innovation.

Dewey, J. (1922). Human Nature and Conduct. The Modern Library.

Donohoe, M., Fletton, J., Hook, A., Powell, R., Robinson, I., Stead, J., Sweeney, K., Taylor, R., \& Tooke, J. (2000). Improving foot care for people with diabetes mel- 
litus. A randomized controlled trial of an integrated care approach. Diabetic Medicine, 17, 581-587.

Donselaar, C., De Boer, D., Van der Hoek, L., Krol, M., Rademakers, J., \& Delnoij, D. (2009). Ervaringen van verzekerden met de zorg en de zorgverzekeraars. CQ-index Zorg en Zorgverzekering, meting 2009. Nivel.

Drees, M., Wroten, K., Smedley, M., Mase, T., \& Schwartz, S. (2015). Carrots and sticks. Achieving high healthcare personnel influenza vaccination rates without a mandate. Infection Control \& Hospital Epidemiology, 36, 717-724.

Drewes, H., Heijink, R., Struijs, J., \& Baan, C. (2014). Landelijke monitor populatiemanagement. Rijksinstituut voor Volksgezondheid en Milieu.

Drews, F., Wallace, J., Benuzillo, J., Markewitz, B., \& Samore, M. (2012). Protocol adherence in the intensive care unit. Human Factors and Ergonomics in Manufacturing \& Service Industries, 22, 21-31.

Engeström, Y., \& Blackler, F. (2005). On the life of the object. Introduction. Organization, 12(3), 307-330.

Enthoven, A., \& Van de Ven, W. (2007). Going Dutch. Managed-competition health insurance in the Netherlands. The New England Journal of Medicine, 357(24), 2421-2423.

Epstein, R., \& Street, R. (2011). The values and value of patient-centered care. Annals of Family Medicine, 9(2), 100-103.

doi.org/10.1370/afm.1239.atient-centered

Frampton, S., Guastello, S., Brady, C., Hale, M., Bennett Smith, S., \& Stone, S. (2008). Patient-centered care improvement guide. Institute for Health Care Improvement.

Funnell, M. (2016). Patient empowerment. What does it really mean? Patient Education and Counseling, 99(12), 1921-1922.

doi.org/10.1016/j.pec.2016.10.010

Gaboury, I., Corriveau, H., Boire, G., Cabana, F., Beaulieu, M., Dagenais, P., Gosselin, S., Bogoch, E., Rochette, M., Filiatrault, J., Laforest, S., Jean, S., Fansi, A., Theriault, D., \& Burnand, B. (2013). Partnership for fragility bone fracture care provision and prevention program (P4Bones). Study protocol for a secondary fracture prevention pragmatic controlled trial. Implementation Science, 8(10).

Gerson, E., \& Star, S. (1986). Analyzing due process in the workplace. ACM Transactions and Information Systems, 4, 257-270.

Gherardi, S. (2000). Practice-based theorizing on learning and knowing in organizations. Organization, 7(2), 211-223.

Gherardi, S. (2014). How to conduct a practice-based study. Problems and methods. Edward Elgar Publishing Ltd. 
Gieryn, T. (1983). Boundary-work and the demarcation of science from non-science. Strains and interests in professional ideologies of scientists. American Sociological Review, 48(6), 781-795.

Glimmerveen, L., Nies, H., \& Ybema, S. (2019). Citizens as active participants in integrated care. Challenging the field's dominant paradigms. International Journal for Integrated Care, 19(1), 6.

doi.org/10.5334/ijic.4202

Greenfield, G., Foley, K., \& Majeed, A. (2016). Rethinking primary care's gatekeeper role. BMJ (Online), 354 (September), 1-6.

doi.org/10.1136/bmj.i4803

Gucciardi, E., Espin, S., Morganti, A., \& Dorado, L. (2016). Exploring interprofessional collaboration during the integration of diabetes teams into primary care. BMC Family Practice, 17, 12.

doi.org/10.1186/s12875-016-0407-1

Gusfield, J. (1981). The culture of public problems. Drinking-driving and the symbolic order. University of Chicago Press.

Hacking, I. (2002). 'Style' for historians and philosophers. In I. Hacking (Ed.), Historical ontology, (pp1-20). Harvard University Press. 1-20.

Hacking, I. (1983). Representing and intervening. Introductory topics in the philosophy of natural science. Cambridge University Press.

Haidt, J. (2001). The emotional dog and its rational tail. A social intuitionist approach to moral judgment. Psychological Review, 108(4), 814-834.

Håkansson, J., Holmström, I. K., Kumlin, T., Kaminsky, E., Skoglund, K., Höglander, J., Sundler, A., Condén, E., Meranius, M. (2019). “Same same or different ?” A review of reviews of person-centered and patient-centered care. Patient Education and Counseling, 102(1), 3-11.

doi.org/10.1016/j.pec.2018.08.029

Hampson, I., \& Junor, A. (2005). Invisible work, invisible skills. Interactive customer service as articulation work. New Technology, Work and Employment, 20(2), $166-181$.

doi.org/10.1111/j.1468-005X.2005.00151.x

Haselton, M., Nettle, D., \& Andrews, P. (2015). The evolution of cognitive bias. In D.M. Buss (Ed.), The handbook of evolutionary psychology, (pp. 724-746). Wiley. doi.org/10.1002/9780470939376.ch25

Hausman, D., \& Welch, B. (2010). Debate. To nudge or not to nudge. Journal of Political Philosophy, 18(1), 123-136.

doi.org/10.1111/j.1467-9760.2009.00351.x

Heidegger, M. (1962). Being and time. Harper \& Row. 
HRSA. (2020). National survey of organ donation. Attitudes and practices, 2019. Rockville.

Hudon, C., Fortin, M., Haggerty, J., Lambert, M., \& Poitras, M. (2011). Measuring patients' perceptions of patient-centered care. A systematic review of tools for family medicine. Annals of Family Medicine, 9(2), 155-164.

doi.org/10.1370/afm.1226.INTRODUCTION

Hughes, J., Bamford, C., \& May, C. (2008). Types of centredness in health care. Themes and concepts. Medicine, Health Care and Philosophy, 11(4), 455-463. doi.org/10.1007/s11019-008-9131-5

Iedema, R., Carroll, K., Collier, A., Mesman, J., \& Wyer, M. (2019). Video-reflexive ethnography in health research and healthcare improvement. CRC Press.

Iedema, R., Mesman, J., \& Carroll, K. (2013). Visualising health care practice improvement, innovation from within. Radcliffe.

Ishikawa, H., Hashimoto, H., \& Kiuchi, T. (2013). The evolving concept of "patient-centeredness" in patient-physician communication research. Social Science and Medicine, 96, 147-153.

doi.org/10.1016/j.socscimed.2013.07.026

Johnson, K., Patterson, B., Ho, Y., Chen, Q., Nian, H., Davison, C., Slagle, J., Mulvaney, S. (2016). The feasibility of text reminders to improve medication adherence in adolescents with asthma. Journal of the American Medical Informatics Association, 23(3), 449-455.

doi.org/10.1093/jamia/ocv158

Kaasa, S., Loge, J., Aapro, M., Albreht, T., Anderson, R, Bruera, E., Caraceni, A., Cervantes, A., Currow, D., Deliens, L., Fallon, M., Gómez-Batiste, X., Grotmol, K., Hannon, B., Haugen, D., Higginson, I., Hjermstad, M., Hui, D., Jordan, K., ... Lundeby, T. (2018). Integration of oncology and palliative care. A Lancet Oncology Commission. The Lancet Oncology, 19(11), e588-e653.

doi.org/10.1016/s1470-2045(18)30415-7

Kahneman, D. (2011). Thinking, fast and slow. Penquin Books.

Kant, I. (2007). Kritiek van de zuivere rede (J. Veenbaas \& W. Visser, Trans.). Boom. (Original work published 1781)

Katz, C. (2012). Integrated approach to outpatient dental treatment of a patient with cerebral palsy. A case report. Special Care in Dentistry, 32(5), 210-217. doi.org/10.1111/j.1754-4505.2012.00267.x

Keshet, Y., Ben-Arye, E., \& Schiff, E. (2013). The use of boundary objects to enhance interprofessional collaboration. Integrating complementary medicine in a hospital setting. Sociology of Health and Illness, 35(5), 666-681.

doi.org/10.1111/j.1467-9566.2012.01520.x 
Kodner, D. L., \& Spreeuwenberg, C. (2002). Integrated care. Meaning, logic, applications, and implications. A discussion paper. International Journal of Integrated Care, 2, 1-8.

Kreindler, S. (2013). The politics of patient-centred care. Health Expectations, 18, $1139-1150$.

doi.org/10.1111/hex.12087

Kümpers, S., Mur, I., Hardy, B., Maarse, H., \& Van Raak, A. (2006). The importance of knowledge transfer between specialist and generic services in improving health care. A cross-national study of dementia care in England and The Netherlands. The International Journal of Health Planning and Management, 21(2), 151-167. doi.org $/ 10.1002 / \mathrm{hpm} .837$

Lafaye, C., \& Thevenot, L. (1993). Une justification ecologique? Revue Francaise de Sociologie, 34(4), 495-524.

doi.org/10.2307/3321928

Langberg, E., Dyhr, L., \& Davidsen, A. (2019). Development of the concept of patient-centredness. A systematic review. Patient Education and Counseling, 102(7), 1228-1236.

doi.org/10.1016/j.pec.2019.02.023

Langley, A., Lindberg, K., Mørk, B., Nicolini, D., Raviola, E., \& Walter, L. (2019). Boundary work among groups, occupations, and organizations. From cartography to process. Academy of Management Annals, 13(2), 704-736.

doi.org/10.5465/annals.2017.0089

Latour, B. (1987). Science in action. How to follow scientists and engineers through society. Harvard University Press.

Latour, B. (1988). The pasteurization of France. Harvard Unversity Press.

Latour, B. (1999). Pandora's hope. Essays on the reality of science studies. Presidents and Fellows of Harvard College.

Latour, B., \& Woolgar, S. (1979). Laboratory life. SAGE Publications.

Lodge, M., \& Wegrich, K. (2016). The rationality paradox of nudge. Rational tools of government in a world of bounded rationality. Law \& Policy, 38(3), 250-267. doi.org/10.1111/lapo.12056

Luxford, K., Safran, D., \& Delbanco, T. (2011). Promoting patient-centered care. A qualitative study of facilitators and barriers in healthcare organizations with a reputation for improving the patient experience. International Journal for Quality in Health Care, 23(5), 510-515.

May, C. (2013). Agency and implementation. Understanding the embedding of healthcare innovations in practice. Social Science \& Medicine, 78, 26-33.

doi.org/10.1016/j.socscimed.2012.11.021 
May, Carl. (2007). The clinical encounter and the problem of context. Sociology, 41(1), 29-45.

Mead, N., \& Bower, P. (2000). Patient-centredness. A conceptual framework and review of the empirical literature. Social Science \& Medicine, 51(7), 1087-1110.

Merriam-Webster. (2021). articulate. In Merriam-Webster.com dictionary.

www.merriam-webster.com/dictionary/articulate

Mesman, J. (2009). The geography of patient safety. A topical analysis of sterility. Social Science \& Medicine, 69(12), 1705-1712.

doi.org/10.1016/j.socscimed.2009.09.055

Mesman, J. (2011). Resources of strength. An exnovation of hidden competences to preserve patient safety. In E. Rowley \& J. Waring (Eds.), A socio-cultural perspective on patient safety, (pp. 71-92. Ashgate.

Milam, P. (2018). Nudging and design for moral responsibility. Nudging and Moral Responsibility Workshop. Amsterdam.

Mohnen, S., Molema, C., Steenbeek, W., Van den Berg, M., de Bruin, S., Baan, C., \& Struijs, J. (2017). Cost variation in diabetes care across Dutch care groups? Health Services Research, 52(1), 93-112.

doi.org/10.1111/1475-6773.12483

Mol, A. (2003). The body multiple. Duke University Press.

Mol, A. (2008). The logic of care. Health and the problem of patient choice. Routledge.

Moore, L., Britten, N., Lydahl, D., Naldemirci, O., Elam, M., \& Wolf, A. (2017).

Barriers and facilitators to the implementation of person-centred care in different healthcare contexts. Scandinavian Journal of Caring Science, 31 (4), 662-673.

doi.org/10.1111/scs.12376

Navin, M. (2017). The ethics of vaccination nudges in pediatric practice. HEC Forum, 29(1), 43-57. doi.org/10.1007/s10730-016-9311-2

NHS Engeland. (2016). New care model. Vanguards - developing a blueprint for the future of NHS and care services.

Nicolini, D. (2013). Practice theory, work, and organization. An introduction. Oxford University Press.

Oldenhof, L., Postma, J., \& Putters, K. (2014). On justification work. How compromising enables public managers to deal with conflicting values. Public Administration Review, 74(1), 52-63.

Oliver, A. (2013). From nudging to budging. Using behavioural economics to inform public sector policy. Journal of Social Policy, 42(04), 685-700. doi.org/10.1017/s0047279413000299

Osmundsen, T. C., Dahl, U., \& Kulseng, B. (2019). Enhancing knowl- 
edge and coordination in obesity treatment. A case study of an innovative educational program. BMC Health Services Research, 19(1), 278. doi.org/10.1186/s12913-019-4119-9

Pettit, P. (2007). Neuroscience and agent-control. In D. Ross, D. Spurrett, K. Harold, \& G. Stephens (Eds.), Distributed cognition and the will. Individual volition and social context, (pp. 77-91). The MIT Press.

Pickering, A. (1995). The mangle of practice. Time, agency and science. The University of Chicago Press.

Pols, J. (2006). Washing the citizen. Washing, cleanliness and citizenship in mental health care. Culture, Medicine \& Psychiatry, 30(1), 77-104.

Pols, J. (2015). Towards an empirical ethics in care. Relations with technologies in health care. Medicine, Health Care \& Philosophy, 18(1), 81-90.

doi.org/10.1007/s11019-014-9582-9

Pols, J. (2018). Waardigheid in de zorg. Empirisch ethische bevindingen. Dies Natalis, Universiteit Voor Humanistiek. Universiteit voor Humanistiek.

Popper, K. (1959). The logic of scientific discovery. Routledge.

Porter, T. (1995). Trust in numbers. The pursuit of objectivity in science and public life Princeton University Press.

Postma, J., Oldenhof, L., \& Putters, K. (2015). Organized professionalism in healthcare. Articulation work by neighbourhood nurses. Journal of Professions and Organization, 2(1), 61-77.

doi.org/10.1093/jpo/jou008

Quick, K., \& Feldman, M. (2014). Boundaries as junctures. Collaborative boundary work for building efficient resilience. Journal of Public Administration Research and Theory, 24(3), 673-695.

RIVM. (2018). Public health foresight study 2018. A healthy prospect. Bilthoven.

Reach, G. (2016). Patient education, nudge, and manipulation. Defining the ethical conditions of the person-centered model of care. Patient Preference and Adherence, $10,459-468$.

doi.org/10.2147/PPA.S99627

Reckwitz, A. (2002). Toward a theory of social practices. A development in culturalist theorizing. European Journal of Social Theory, 5(2), 243-263.

Roe, A., Yapalater, S., \& Hadjiliadis, D. (2020). Electronic health nudges to improve reproductive health care for women with cystic fibrosis. Journal of Cystic Fibrosis, 20(3), 11-12.

doi.org/10.1016/j.jcf.2020.11.004

Rouse, J. (2001). Two concepts of practices. In T. Schatzki, K. Knorr-Cetina, \& E. Von Savigni (Eds.), The practice turn in contemporary theory (pp. 198-208). 
Routledge.

Ruwaard, D., Spreeuwenberg, M., Kroese, M., Steevens, J., Ouwens, M., Hilbink, M., \& Braspenning, J. (2014). Triple Aim en de toepassing in de regionale proeftuinen "Populatiemanagement" van VGZ en CZ. Vakgroep Health Services Research, Maastricht University \& IQ healthcare, Radboud University Nijmegen Medical Centre.

Savel, R. H., \& Munro, C. L. (2015). Moral distress, moral courage. AJCC American Journal of Critical Care, 24(4), 276-278.

doi.org/10.4037/ajcc2015738

Sayer, A. (2011). Why things matter to people. Social science, values and ethical life. Cambridge University Press.

Schatzki, T. (2001a). Introduction. In T. Schatzki, K. Knorr-Cetina, \& E. Von Savigny (Eds.), The practice turn in contemporary theory (pp. 10-23). Routledge.

Schatzki, T. (2001b). Practice minded orders. In T. Schatzki, K. Knorr Cetina, \& E. Von Savigni (Eds.), The practice turn in contemporary theory (pp. 50-63). Routledge.

Schiavone, G., De Anna, G., Mameli, M., Rebba, V., \& Boniolo, G. (2014). Libertarian paternalism and health care policy. A deliberative proposal. Medicine, Health Care \& Philosophy, 17(1), 103-113.

doi.org/10.1007/s11019-013-9502-4

Schön, D., \& Rein, M. (1995). Frame reflection. Toward the resolution of intractable policy controversies. Basic Books.

Schulpen, G. (2003). The joint consultation of general practitioners and rheumatologists. Maastricht Universtity, Maastricht.

Schulpen, G., Meerlo, R., Van Uden, C., Dekkers, T., Van Hees, A., \& Maes, J. (2012). Blauwe Zorg. Regio-experiment voor duurzame zorg in Maastricht en Heuvelland. ZIO, VGZ \& Huis voor de Zorg.

www.zio.nl/workspace/uploads/blauwe-zorg-oktober-2012-509cd46146406.pdf

Selinger, E., \& Whyte, K. (2010). Competence and trust in choice architecture. Knowledge, Technology \& Policy, 23(3-4), 461-482. doi.org/10.1007/s12130-010-9127-3

Sharon, T. (2018). When digital health meets digital capitalism, how many common goods are at stake? Big Data \& Society, 5(2), 1-12. doi.org/10.1177/2053951718819032

Sie, M. (2012). Keuze-architectuur of individuale verantwoordelijkheid? Een filosofische bijdrage. In M. Luckerath-Rovers, H. Bier, M. Van Hees, \& M. Kaptein (Eds.), (171-183). Jaarboek Corporate Governance 2012-2013. Kluwer.

Sie, M. (2014). Self-knowledge and the minimal conditions of responsibility. 
A traffic-participation view on human (moral) agency. The Journal of Value Inquiry, 48(2), 271-291.

doi.org/10.1007/s10790-014-9424-2

Sixma, H., Kerssens, J., Campen, C., \& Peters, L. (1998). Quality of care from the patients' perspective. From a theoretical concept to a new measuring instrument. Health Expectations, 1(2), 82-95.

doi.org/10.1046/j.1369-6513.1998.00004.x

Soler, R, Proia, K., Jackson, M., Lanza, A., Klein, C., Leifer, J., \& Darling, M. (2018).

Nudging to change. Using behavioral economics theory to move people and their health care partners toward effective type 2 diabetes prevention. Diabetes Spectrum, 31(4), 310-319.

doi.org/10.2337/ds18-0022

Star, S., \& Griesemer, J. (1989). Institutional ecology, "translations" and boundary objects. Amateurs and professionals in Berkeley's Museum of Vertebrate Zoology, 1907-39. Social Studies of Science, 19(3), 387-420.

Steenkamer, B., Drewes, H., Heijink, R., Baan, C., \& Struijs, J. (2016). Defining population pealth management. A scoping review of the literature. Population Health Management, 20(1), 74-85.

Strauss, A. (1985). Work and the division of labor. Sociological Quarterly, 26(1), $1-19$.

Strauss, A. (1988). The articulation of project work. An organizational process. The Sociological Quarterly, 29(2), 163-178.

Sullivan, H., \& Williams, P. (2012). Whose kettle? Exploring the role of objects in managing and mediating the boundaries of integration in health and social care. Journal of Health, Organisation and Management, 26(6), 697-712. doi.org/10.1108/14777261211276970

Sunstein, C. (2014). Nudging. A very short guide. Journal of Consumer Policy, 37, 583-588.

Sunstein, C., \& Ullman-Margalit, E. (1999). Second-decisions. Ethics, 110(1), 5-31.

doi.org/10.1086/233202

Swidler, A. (2001). What anchors cultural practices? In T. Schatzki, K. Knorr-Cetina (Ed.), The practice turn in contemporary theory (pp. 83-101). Routledge.

Swierstra, T. (1992). Latour de force. Kennis en methode, 1, 21-38.

Swierstra, T. (2002). Ethiek op zijn plaats zetten. Krisis, 3(3), 18-38.

Swierstra, T. (2015). Identifying the normative challenges posed by technology's 'soft' impacts. Etikk i Praksis. Nord Journal of Applied Ethics, 9(1), 5-20.

Szerszynski, B., \& Galarraga, M. (2013). Geoengineering knowledge. Interdiscipli- 
narity and the shaping of climate engineering research. Environment and Planning A: Economy and Space, 45(12), 2817-2824.

doi.org/10.1068/a45647

Taylor, K. (2009). Paternalism, participation and partnership. The evolution of patient centeredness in the consultation. Patient Education and Counseling, 74, $150-155$.

doi.org/10.1016/j.pec.2008.08.017

Thaler, R., \& Sunstein, C. (2008). Nudge. Improving decisions about health, wealth and happiness. Penquin Books.

Thevenot, L. (2001). Pragmatic regimes governing the engagement with the world. In K. Knorr-Cetina, T. Schatzki E. Von Savigny (Ed.), The Practice Turn in Contemporary Theory, (pp.64-82). Routledge.

Thevenot, L., Moody, M., \& Lafaye, C. (2000). Forms of valuing nature. Arguments and modes of justification in French and American environmental disputes. In Rethinking Comparative Cultural Sociology. Repertoires of Evaluation in France and the United States, (pp. 229-272). Cambridge University Press.

Timmermans, S., \& Berg, M. (2003). The gold standard. The challenge of evidence based medicine and standardization in health care. Temple University Press.

Tversky, A., \& Kahneman, D. (1974). Judgment under uncertainty. Heuristics and biases. Biases in judgments reveal some heuristics of thinking under uncertainty. Science, 185(4157), 1124-1131.

Van Campen, C., Sixma, H., Kerssens, J., \& Peters, L. (1998). Assessing non-institutionalized asthma and COPD patients' priorities and perceptions of quality of health care. The development of the QUOTE-CNSLD instrument. Journal of Asthma, 34(6), 531-538.

Van Campen, C., Sixma, H., Kerssens, J., Peters, L., \& Rasker, J. (1997). Assessing patients' priorities and perceptions of the quality of health care. The development of the QUOTE-rheumatic-patients instrument. The British Journal of Rheumatology, 37, 362-368.

Van de Werff, T. (2018). Practicing the plastic brain. Popular neuroscience and the good life [Unpublished doctoral dissertation]. Maastricht University.

Van Damme, E. (2012). Concurrentie en samenwerking op de zorgverleningsmarkt. In F. Schut \& M. Varkevisser (Eds.), Een economisch gezonde gezondheidszorg. Sdu Uitgevers.

Van de Ven, W., \& Schut, F. (2008). Universal mandatory health insurance in the Netherlands. A model for the United States? Health Affairs (Millwood), 27(3), 771-781.

doi.org/10.1377/hlthaff.27.3.771 
Van den Bogaart, E., Quanjel, T., Kroese, M., Spreeuwenberg, M., Hameleers, N., Westra, D., \& Ruwaard, D. (2019). Monitoring en evaluatie van substitutie van zorg in drie Limburgse proeftuinen. Maastricht.

Van Hoof, S., Kroese, M., Spreeuwenberg, M., Elissen, A., Meerlo, R., Hanraets, M., \& Ruwaard, D. (2016). Substitution of hospital care with primary care. Defining the conditions of Primary Care Plus. International Journal of Integrated Care, 16(1), 12 .

doi.org/10.5334/ijic.2446

Van Hoof, S., Spreeuwenberg, M., Kroese, M., Steevens, J., Meerlo, R., Hanraets, M., \& Ruwaard, D. (2016). Substitution of outpatient care with primary care. A feasibility study on the experiences among general practitioners, medical specialists and patients. BMC Family Practice, 17, 108. doi.org/10.1186/s12875-016-0498-8

Van Rhijn, L., Spreeuwenberg, M., \& Ruwaard, D. (2013). Orthopedie gebaat bij anderhalvelijnszorg. Medisch Contact, 68(5), 283-285.

Vanhaecht, K., \& Sermeus, W. (2002). Draaiboek voor de evaluatie van een klinisch pad. Acta Hospitalia, 3, 13-27.

Vargas, M. (2013). Building better beings. Oxford University Press.

Velleman, J. (1992). What happens when someone acts? Mind, New Series, 101(403), 461-481.

Vosman, F., den Bakker, J., \& Weenink, D. (2016). How to make sense of suffering in complex care practices. In G. Spaargaren, D. Weenink, \& M. Lamers (Eds.), Practice theory and research: Exploring the dynamics of social life (pp. 117-130). Routledge.

Webster, A. (2006). Introduction. New technologies in health care. Opening the black bag. In A. Webster (Ed.), New Technologies in Health Care. Challenge, Change and Innovation (pp. 1-10). Palgrave Macmillan.

doi.org/10.1177/0002764294038002008

Whyte, K., Selinger, E., Caplan, A., \& Sadowski, J. (2012). Nudge, nudge, or shove, shove. The right way for nudges to increase the supply of donated cadaver organs. The American Journal of Bioethics, 12(2), 32-39.

Wiles, R. (1997). Empowering practice nurses in the follow-up of patient with established heart disease. Lessons from patients' experiences. Journal of Advanced Nursing, 26, 729-735.

Williams, A., Liu, P., Muir, K., \& Waxman, E. (2018). Behavioral economics and diabetic eye exams. Preventive Medicine, 112(December 2017), 76-87. doi.org/10.1016/i.ypmed.2018.04.006

Zaslavsky, A., Zaborski, L., \& Cleary, P. (2002). Factors affecting respons rates 
to the Consumer Assessment of Health Plans Study Survey. Medical Care, (40), 485-489.

Zaslavsky, A., Zaborski, L., Ling Ding, M., Shaul, J., \& Cioff, P. (2001). Adjusting performance measures to ensure equitable plan comparisons. Health Care Finance Review, (22), 109-126.

Zuiderent-Jerak, T. (2015). Situated Intervention. Sociological experiments in health care. MIT Press. 


\section{SAMENVATIING}

\section{DOOR GEWRICHTEN VERBONDEN HET KALIBREREN VAN KENNIS, MORAAL EN GEZONDHEIDSZORG}

Marktwerking in de Nederlandse zorg staat alweer jaren ter discussie. Veel van de kritiek komt in de kern op het volgende neer: marktrelaties verschillen (in moreel opzicht) zo fundamenteel van zorgrelaties dat wanneer we de zorg als markt organiseren dit tot (moreel) problematisch gedrag en verhoudingen leidt. In deze dissertatie staat een proeftuin in de zorg centraal, die is opgezet vanuit de overtuiging dat het niet de markt is die de zorg efficiënter gaat maken. De proeftuin, waarin een nationale zorgverzekering, een eerstelijns zorgbedrijf, een lokaal ziekenhuis en een patiëntenorganisatie samen interventies uitvoeren, pleit juist voor belangeloze samenwerking als de weg naar duurzaamheid. De proeftuin noemt zichzelf Blauwe Zorg. Zoals de kleur groen duurzaamheid in onze omgang met brandstoffen en andere milieuvraagstukken op de kaart heeft gezet, zo willen de initiatiefnemers van de proeftuin dat zorgverleners, patiënten en managers blauw gaan associëren met duurzaamheid in de zorg. Samenwerking maakt het systeem efficiënter en gaat verkwistend individueel gedrag tegen, is het idee. Zo kunnen we kosten besparen in de zorg zonder dat dit ten koste gaat van kwaliteit en toegankelijkheid.

Het proefschrift volgt de meest omvangrijke interventie van Blauwe Zorg: anderhalvelijnszorg, verleend op de nieuw geopende stadspoli's. Deze consultaties zijn bedoeld voor patiënten met laag-complexe medische problemen, die de huisarts anders naar een specialist in het ziekenhuis verwezen zou hebben. Op de stadspoli kunnen deze patienten een 'meekijkconsult' met een specialist plannen. De initiatiefnemers presenteren de interventie doorgaans met drie beloften. Ten eerste, door de intensievere samenwerking tussen huisarts en medisch specialist zullen ze van elkaar leren. De tweede belofte is dat het verplaatsen van medisch specialistische zorg naar de buurt de zorg toegankelijker zal maken. Ten derde zullen er kosten bespaard worden. Patiënten en specialisten zullen op een eerstelijnslocatie in vergelijking tot in het ziekenhuis minder geneigd zijn besluiten te nemen die niet direct medisch noodzakelijk zijn maar wel kostbaar. Deze drie beloftes belicht ik vanuit twee verschillende gezichtspunten. Eerst plaats ik ze in een breder (gezondheids)wetenschappelijk kader, dan bekijk ik minutieus hoe de beloften uitpakken in 
de praktijk. Op basis van een analyse van het contrast tussen de twee, formuleer ik in dit proefschrift niet alleen een kritisch-constructieve visie op de interventie zelf, maar ook op de drie achterliggende (gezondheids)wetenschappelijke trends waarmee de beloftes verbonden zijn, en de manier waarop dit soort interventies meestal geëvalueerd worden.

In het inleidende hoofdstuk plaats ik de proeftuin en interventie in de bredere context van het proeftuinenbeleid en bevraag ik de evaluatiemethoden van dit beleid. Ik presenteer een kritisch alternatief voor de bestudering van zorginnovaties en zet de theoretische achtergrond van zo een benadering uiteen.

Als onderdeel van het proeftuinenbeleid van het Ministerie van Volksgezondheid, Welzijn en Sport onderzocht het RIVM, het wetenschappelijke onderzoeksinstituut van het ministerie, in samenwerking met een team van gezondheidswetenschappers, de stadspoli aan de hand van het Triple Aim, later Quadruple Aim, principe. Deze benadering vertrekt vanuit de overtuiging dat we alleen hoogwaardige gezondheidszorg kunnen leveren als we ons op drie verschillende doelen (verbetering van de ervaring van patiënten, kostenreductie, gezondheidstoename en tevredenheid van zorgverleners) tegelijkertijd richten. Om te onderzoeken in hoeverre de interventies hun doelen bereikten, richten de onderzoekers zich op uitkomstmaten verzameld met vragenlijsten.

Een interventie beoordelen door naar de uitkomstmaten te kijken, past binnen het idee van de "proeftuin". Een proeftuin is een locatie waar zorgorganisaties kunnen experimenteren met nieuwe vormen van zorgverlening. Een experiment is dan een systematische procedure om een hypothese te testen, waarbij het van belang is de effecten van een verandering te meten terwijl andere factoren gelijk blijven. De hypothese is dat het verplaatsen van zorg tot de drie bovengenoemde uitkomsten leidt. Om dit vast te kunnen stellen, moeten de uitkomsten (interventiegroep) vergeleken worden met uitkomsten van vergelijkbaar medisch specialistische zorg verleend in het ziekenhuis (controlegroep). Zo kunnen onderzoekers zicht krijgen op welke effecten toegeschreven kunnen worden aan de interventie zelf.

Deze benadering heeft een tweetal beperkingen. Een test komt met vooraf gedefinieerde criteria. Daarmee reduceert het de interventie tot haar uitkomstmaten en neemt dus dat wat er gebeurde tijdens de interventie uit beeld. Want wat gebeurde er nou eigenlijk tijdens de anderhalvelijnszorgconsultaties? Hoe gaven medisch specialisten binnen de beperkingen van de locatie vorm aan dit nieuwe type zorg? Hoe ging men om met onverwachte problemen? Een andere beperking is de methode van dataverzameling, cruciaal voor het vergelijken van interventie- en controlegroep. Om dit te kunnen doen moeten de verzamelde ervaringen zoveel mogelijk 
gestandaardiseerd worden. Dit gebeurt met vragenlijsten waarmee de expliciete kennis van dokters en patiënten verzameld wordt. Veel mensen, in dit geval professionals en patiënten, zijn echter blind voor de vele gewoontes die hun dagelijkse bezigheden vormgeven. Veel van onze waarnemingen en acties volgen impliciete routines. Als we expliciete kennis en ervaringen verzamelen met vragenlijsten blijft veel impliciete kennis buiten beeld.

In het proefschrift hanteer ik de methode van exnovatie. Door niet naar uitkomsten van de zorg te kijken, maar juist naar haar alledaagse processen om daarop te reflecteren en ervan te leren, breng ik juist ook de impliciete gewoontes in beeld. Wat doen managers, dokters en patiënten dagelijks als ze anderhalvelijnszorg organiseren, leveren en/of ontvangen? Met deze praktijkkennis wordt het mogelijk te bepalen hoe anderhalvelijnszorg verbeterd kan worden, wat de oorzaak is als het ineens minder loopt, of welke aspecten van de interventie wel en niet verplaatst kunnen worden.

Exnovatie is onderdeel van de praktijkwending die zich in de tweede helft van de 20e eeuw voltrokken heeft in de sociale en geesteswetenschappen. Deze wending stelt de activiteiten die tezamen een praktijk (re)produceren centraal. Ik bouw voort op het werk van Pickering wiens onderzoek naar de wetenschappelijke praktijk ook onderdeel is van de praktijkwending. Hij zet zich af tegen wat hij het idioom van representatie noemt. Deze manier van spreken over de wetenschap vooronderstelt dat de wetenschap een geheel aan kennis is dat de wereld representeert. Wetenschap bedrijven is binnen dit idioom de set van activiteiten gericht op het steeds representatiever maken van dit geheel aan kennis. Het performatieve idioom dat Pickering als alternatief voorstelt, heft het fundamentele onderscheid tussen materiele wereld enerzijds en representatieve kennis over die wereld anderzijds op. Beiden zijn onderdeel van één speelveld waarin mensen, kennis en objecten voortdurend onvoorspelbaar handelen: ze hebben een impact op de wereld omdat ze steeds relaties met zichzelf en de wereld aangaan.

In de dissertatie bekijk ik de anderhalvelijnszorg vanuit een performatiefidioom. Ik beantwoord de vraag hoe anderhalvelijnszorg gedaan wordt in de dynamische en heterogene praktijk waarin voortdurend werk verricht wordt om organisatie en zorgverlening goed te laten verlopen. Dit doe ik aan de hand van de data verzameld met een etnografisch onderzoek dat liep van 2015 tot 2018: documentanalyse, interviews met medisch specialisten, patiënten, initiatiefnemers en managers en observaties van vergaderingen en consultaties op de stadspoli, in huisartspraktijken en op de ziekenhuispoli's. 
In het tweede hoofdstuk beschrijf ik elk van de drie beloftes en plaats ik deze in een (gezondheids)wetenschappelijk kader. Ik laat zien welke noties van handelingsvermogen, kennis en/of moraliteit de beloftes vooronderstellen. Dit vormt de basis voor de rest van de dissertatie, waarin ik de beloftes vanuit een performatief idioom bestudeer.

De belofte dat door samenwerking de kennis van zowel huisartsen als specialisten zal toenemen past in een wereldwijde trend in de organisatie van gezondheidszorg: Integrated Care. Binnen deze trend wordt het overbruggen van verschillen en opheffen van fragmentatie in de zorg gezien als het middel om zorgsystemen efficienter te maken en de kwaliteit van zorg te verbeteren. Integrated Care, en dus ook de eerste belofte, is opgesteld in het idioom van representatie. Door jarenlange processen van subspecialisatie in de medische wereld is medische kennis gefragmenteerd geraakt. Integrated Care omvat activiteiten als de verticale integratie van anderhalvelijnszorg, die als doel hebben deze fragmentatie tegen te gaan door professionals samen te laten werken. Dit vooronderstelt dat kennis een goed is, een product, of arbeid, dat iemand bezit. Kennis kan dan overgedragen worden, en onproblematisch verplaatst van de ene naar de andere praktijk en/of professional.

Volgens de tweede belofte maakt de verplaatsing naar de buurt de zorg toegankelijker, en dus patiëntvriendelijker. Dit past binnen een beweging in de gezondheidswetenschappen die de patiënt en haar voorkeuren centraal stelt: patiëntgerichte zorg. De vele artikelen over het onderwerp wijzen er vaak op dat het begrip onduidelijk is gedefinieerd en daardoor moeilijk is te onderzoeken en te implementeren. Hierop volgt dan vaak een aangescherpte definitie of een voorstel om het in bepaalde dimensies op te delen. Dit zijn pogingen om een valide representatie te vinden van zorg die daadwerkelijk patiëntgericht is. De onderzoekers trachten dan bijvoorbeeld instrumenten te ontwikkelen waarmee vastgesteld kan worden of geleverde zorg voldoet aan een objectieve standaard of essentie van patiëntgerichtheid. Kortom, zulk onderzoek is opgesteld in het idioom van representatie. Zulk onderzoek, echter, is niet alleen gericht op het steeds representatiever maken van kennis erover, maar ook op het patiëntgerichter maken van de zorg zelf. In die zin gaat het hier dus niet alleen om kennis als representatie, maar ook om moraliteit als representatie. Patiëntgerichte zorg is dan een meetbare eigenschap van verleende zorg en daarmee een ethisch goed dat al dan niet voldoende aanwezig is in een bepaalde instantie van zorgverlening. Bij moraliteit als representatie gaat het er ook om dat de uitgevoerde handelingen een ideaal voldoende representeert. 
De derde belofte is dat anderhalvelijnszorg een kostenbesparing oplevert. De veranderde omgeving maakt dat patient en medisch specialist anders naar medische problemen kijken en daardoor minder vaak verkwistende besluiten nemen. Deze claim sluit aan bij het beleidsinstrument van de nudge, het aanpassen van de keuze-architectuur met het doel gedrag te veranderen zonder opties te verbieden of economische prikkels toe te passen. Nudging is gebaseerd op de dual process theory die uitgaat van twee verschillende systemen van handelen. Systeem één is het routineuze handelen dat we meestal doen zonder erbij na te denken. Zulk handelen is gesitueerd, dat wil zeggen, de acties zijn directe reacties op onze omgeving. Als we handelen op basis rationele overwegingen, dan gebruiken we systeem twee, het deliberatieve systeem. Nudging gaat uit van de gedachte dat het makkelijker is het automatisch handelen van mensen te veranderen door de omgeving aan te passen, dan door met informatie of advies invloed te hebben op systeem twee. Een belangrijk bezwaar tegen het gebruik van nudging (in de gezondheidszorg) is dat het de rationele capaciteiten van degenen die genudget worden omzeilt. Voorstanders van nudging hebben hierop twee antwoorden. Ten eerste is een nudge niet meer dan een subtiel duwtje; je kan altijd besluiten iets anders te doen. Ten tweede is een nudge een duwtje in de goede richting. Letterlijk: het helpt mensen het besluit te nemen dat juist is volgens hun eigen standaarden. In de dual process theory, ontwikkeld als kritisch alternatief van de rationeel handelende actor, komt dezelfde notie van handelingsvermogen weer door de achterdeur terug. Mensen handelen niet altijd rationeel, maar de richting en legitimiteit van nudges volg nog altijd wel de maatstaf van het rationeel handelen. Nudging en de derde belofte doen dus beroep op de notie van moraliteit als representatie, omdat met een nudge getracht wordt het daadwerkelijk gedrag van mensen in overeenstemming te brengen met hun voorkeuren.

In het derde hoofdstuk staat de eerste belofte van anderhalvelijnszorg centraal. Ik problematiseer de vooronderstellingen van Integrated Care en bestudeer Integrated-Care-in-actie. Dat wil zeggen, in de drie vergaderingen van de gebruikersraad van de stadspoli's die ik bespreek in dit hoofdstuk, kijk ik naar wat mensen doen als ze in het organiseren van anderhalvelijnszorg omgaan met de kennisverschillen van professionals.

Het hoofdstuk start met observaties van een discussie over hoe patiënten met incontinentie doorverwezen zouden moeten worden. Wat blijkt, kennisverschillen worden niet opgelost of afgestemd, maar er wordt juist ruimte voor gemaakt. Dit gaat in tegen de verwachting van Integrated Care dat samenwerken fragmentatie zou tegengaan. Diskwalificeert dit voorbeeld anderhalvelijnszorg als een (succes- 
vol) voorbeeld van Integrated Care? Ik beargumenteer het tegendeel. Als we vanuit een performatief idioom naar deze discussie kijken, dan zien we dat het een voorbeeld is van het voortdurende werk dat betrokkenen in de organisatie verrichten om het managen en verlenen van anderhalvelijnszorg mogelijk te maken. Dit is Integrated-Care-in-actie, dat wil zeggen, het werk nodig om ondanks kennisverschillen zorg te organiseren. De vraag van dit hoofdstuk is: hoe krijgt Integrated-Care-in-actie vorm in deze praktijk?

Op basis van de eerste observaties ontwikkel ik de notie van kenniswerk. Dit is gebaseerd op het concept articulatiewerk, de alledaagse, informele, en vaak onzichtbaar blijvende activiteiten uitgevoerd om organisatieprocessen vlot te laten verlopen. De literatuur maakt een onderscheid tussen haar temporele en spatiale dimensie: om vergaderingen te kunnen houden moeten agenda's afgestemd worden maar ook het gebruik en de inrichting van ruimtes. Ik beargumenteer dat articulatiewerk ook een kennisdimensie heeft. Dit is het werk waarmee kennisverschillen worden overbrugd, op elkaar afgestemd en/of geaccommodeerd, cruciaal voor het vlot verlopen van organisatieprocessen. Er moet niet alleen met stoelen, tafels en afspraken geschoven worden maar ook met stukjes kennis en betekenis.

In de andere twee discussies die ik analyseer wordt een breed scala aan kenniswerk verricht om gestagneerde organisatieprocessen weer op gang te brengen. $\mathrm{Om}$ er een paar te noemen: problemen worden uit elkaar getrokken en geëxpliceerd, om daarna weer bij elkaar gebracht te worden in een nieuwe voorstelling van zaken. Verschillende probleemdefinities worden gecombineerd en tot nieuwe gemaakt, men schakelt van een focus op de inhoud naar procedure en er wordt vaak gebruik gemaakt van kleine punten van overeenkomst die als een soort gewricht tussen de diverse kennispraktijken fungeren en daarmee als flexibele verbindingen functioneren.

De analyse van dit hoofdstuk geeft aanleiding voor twee conclusies. Ten eerste, kenniswerkprocessen voltrekken zich niet binnen één vergadering; het zijn reeksen waarin steeds op verschillende plekken en momenten dezelfde problemen en/of oplossingen besproken en aangepast worden. Zulke processen zijn niet lineair of cumulatief; kennis en begrip groeit, maar kan daarna weer verdwijnen. De tweede conclusie is dat er verschillende logica's zijn. Soms is kenniswerk gericht op integratie van posities en oplossen van conflicten (realisme); in andere gevallen op het vinden van schakelpunten (gewrichten) tussen conflicterende opvattingen door ze in context te plaatsen (contextualisme) of op het maken van ruimte voor verschillen (pragmatisme). De professionals waren geenszins consistent in het volgen van een specifieke logica. Kenniswerk is een kwestie van het voortdurend aanvoelen van anderen: waar zijn mensen bereid mee te bewegen, waar is verzet, welke logica's 
zullen effectief zijn in het navigeren van de spanningen? De logica's van kenniswerk voltrekken zich niet in het hoofd van individuen, ze zijn onderdeel van het speelveld net als de professionals, de indicatielijsten, de stoelen en tafels; ze zijn onlosmakelijk verbonden met de sociale en materiele wereld.

In het vierde hoofdstuk kijk ik naar de tweede belofte: het verplaatsen van medisch specialistische zorg naar de buurt maakt de zorg patiëntgerichter. In dit hoofdstuk problematiseer ik het onderzoek naar patiëntgerichte zorg, en doe een voorstel voor een alternatieve benadering. Op basis van een analyse van vier verschillende stadspoliconsultaties ontwikkel ik een ander begrip van patiëntgerichte zorg.

Het hoofdstuk start met een stadspoliconsultatie waarin een oogarts op een geheel eigen wijze anderhalvelijnszorg verleent aan een patiënt die vervolgens tevreden de spreekkamer verlaat. Het blijkt lastig op basis van een veel gebruikt meetinstrument de patiëntgerichtheid van deze specifieke consultatie te bepalen. De omgang van de arts met de patiënt kan autoritair overkomen, maar ook juist gezien worden als een bewijs van de sensitiviteit van de arts voor het ongemak van de patiënt bij het maken van een eigen keuze. Exnovatie biedt hier uitkomst. Door samen te reflecteren op de interacties is het mogelijk inzicht te krijgen in hoe patiëntgerichtheid vorm krijgt in de praktijk.

In dit hoofdstuk beargumenteer ik dat articulatiewerk ook een morele dimensie heeft. Dit noem ik rechtvaardigingswerk. (Samen) tot het goede besluit komen is een complex proces omdat mensen vaak verschillende overtuigingen hebben over wat het belangrijkste gemeenschappelijk goed is in een situatie. In de context van deze studie zien we iets vergelijkbaars. Mensen verschillen vaak van mening over wat goede zorg behelst. Moet een patiënt bijvoorbeeld benaderd worden als een individu met een specifieke persoonlijkheid of heeft ze als patiënt-burger recht op een objectieve benadering? Om tijdens een consultatie duurzame besluiten te bereiken, dat wil zeggen, besluiten die medisch-inhoudelijk juist zijn maar ook voldoende draagvlak hebben bij dokter en patiënt, wordt rechtvaardigingswerk verricht: morele werelden worden gekalibreerd, er worden schakelpunten gevonden tussen conflicterende opvattingen.

Patiëntgerichtheid blijkt geen kwaliteit te zijn die gemeten kan worden aan de hand van een checklist, of die je kan bereiken door een protocol te volgen; het is een eigenschap van interacties, een routine ingebed in de sociale, morele en materiele contexten waarin specialist en patiënt samen besluiten nemen over de behandeling. Patiëntgerichtheid is de ruimte die een oogpatiënt krijgt om door middel van haar verbeelding zich het ondergaan van een oogoperatie voor te stellen, het is de zorgvuldig ontwikkelde routine van stadspolizorg die de internist gebruikt om patiën- 
ten te helpen die hun lichaam en de zorg wantrouwen. Zelfs als patiëntgerichtheid een andere betekenis heeft voor dokter en patiënt, kan toch op het niveau van het behandelbesluit voldoende overeenstemming gevonden worden dankzij een klein punt van instemming. Patiëntgerichtheid heeft voor sommige artsen een andere betekenis op de standspoli dan in het ziekenhuis.

Het rechtvaardigingswerk verricht in de stadspoliconsultaties betreft een brede variëteit aan interacties. Sommige voltrekken zich tijdens de consultatie, anderen beslaan een langdurig vervaardigingsproces waarin allerlei ervaringen uit het verleden een rol spelen. In sommige analyses was het rechtvaardigingswerk nodig omdat er een spanning tussen de morele opvattingen van patiënt en dokter bleek te zijn, in andere tussen de verschillende contexten waarin zorg verleend werd. Ook in het geval van moreel articulatiewerk viel het op dat er geenszins volledige afstemming van morele overtuigingen nodig is. Een flexibel "gewricht", dat wil zeggen een schakelpunt dat ruimte laat voor de verbonden morele werelden om te bewegen, bleek genoeg te zijn.

Het vijfde hoofdstuk richt zich op de derde belofte: kostenbesparing. Op basis van een analyse van drie consultaties en een vergadering over orthopedische zorg op de stadspoli laat ik niet alleen zien hoe anderhalvelijnszorg als nudge vorm krijgt in de praktijk, maar ook in hoeverre het een legitiem instrument is deze context.

In de literatuur worden voorbeelden van nudges besproken waarin vaak een eenduidig onderscheid tussen wenselijk en onwenselijk gedrag is en waarin omgevingsfactoren van invloed op gedrag gemakkelijk te identificeren zijn. Bijvoorbeeld: het verspreiden van een citrusgeur op toiletten in het ziekenhuis maakt dat dokters vaker hun handen wassen nadat ze naar de wc zijn gegaan. Op de stadspoli is het verschil tussen een wenselijk en niet-wenselijk verwijsbesluit minder eenduidig en blijkt dat de omgevingsfactoren veel diverser zijn.

Om juist de complexiteit van de consultaties op de voorgrond te zetten, gebruik ik ook in dit hoofdstuk de notie van rechtvaardigingswerk om praktijkvoorbeelden te analyseren. De praktijk blijkt op vier manieren te contrasteren met de dual process theory die aan de basis ligt van nudging. Ten eerste is het handelen van specialisten en patiënten tijdens de consultatie weliswaar vaak automatisch, maar niet gedachteloos. Dit is wat Dewey intelligente routines noemt, die gevormd en aangepast worden in een voortdurend reflectieproces. Ten tweede, het handelen van specialisten en patiënten is wellicht gesitueerd, maar niet als een automatische en directe reactie op de omgeving. Hoe het handelen gesitueerd is hangt af van de morele overtuigingen van degene die handelt; het is gemedieerd. Het derde punt is dat met de verplaatsing van zorg, de rationele capaciteiten vaak niet omzeild worden, maar juist 
aangezet. Omdat ze op de stadspoli nog niet vastliggen, is er ruimte om mogelijk veelbelovende nieuwe routines te ontwikkelen.

Het vierde contrast is van toepassing op de dualiteit van nudger en genudgde. In tegenstelling tot de genudgde, een handelend actor met gelimiteerde rationaliteit, lijkt voorondersteld te worden dat er wat betreft de nudger, degene die de nudge bedenkt, geen sprake is van gelimiteerde rationaliteit. De observaties van de vergadering tonen iets anders. Anderhalvelijnszorg blijkt geen voltooid design te zijn, het is werk in uitvoering. Omdat de keuzearchitectuur tijdens het proces van implementatie nog voortdurend aangepast wordt, bijvoorbeeld door te bepalen welke medisch specialisten wel of niet geschikt zijn om er te werken, blijft het onduidelijk of de lage doorverwijscijfers het resultaat zijn van de nudge die "irrationele" dokters assisteert in het maken van rationele beslissingen, of dat het komt doordat de "irrationele" dokters gaandeweg uit de stadspoli gehaald werden?

Op basis van de analyses formuleer ik drie condities van legitimiteit. Ten eerste, het veranderen van de omgeving is juist waardevol, niet omdat het rationele capaciteiten omzeilt, maar omdat het daartoe aanzet. Een belangrijke conditie is dus dat er ook ruimte moet zijn voor de creatieve processen die de nudge teweegbrengt. Ten tweede blijkt de claim dat een nudge slechts een duwtje geeft in de richting van de eigen voorkeuren, cruciaal in de weerlegging van de kritiek op nudging, onhoudbaar. Voorkeuren liggen niet vast, maar worden gevormd in een dynamisch proces waarin specialist en patiënt met elkaar interacteren. De tweede voorwaarde is dat er genoeg ruimte is voor het dynamische karakter van voorkeuren. Ten derde blijkt de claim dat een nudge niet meer is dan een duwtje niet houdbaar. In de spreekkamer heeft de specialist de vrijheid om tegen de nudge in te gaan, maar als hij dat te vaak doet riskeert hij diskwalificatie. Het is dus cruciaal om ook op managementniveau te garanderen dat de nudge een duwtje is en blijft.

In het slothoofdstuk maak ik de balans op. Ik presenteer een systematische set van concepten die inzicht geven in hoe anderhalvelijnszorg verleend en gemanaged wordt in de praktijk. Ik laat zien hoe deze concepten gebruikt kunnen worden in het volgen van innovatieve gezondheidszorgpraktijken waarin samenwerken centraal staat, hoe zulke praktijken verbeterd kunnen worden en hoe we onderwijl leerprocessen kunnen stimuleren.

De belangrijkste conclusie van het proefschrift is dat het organiseren, ontvangen en verlenen van anderhalvelijnszorg met een grote variëteit aan articulatiewerk komt, in zowel haar morele als kennisdimensie. In de spreekkamer en aan de vergadertafel doen patiënten, dokters en managers voortdurend moeite om ondanks kennis- en 
morele verschillen tot duurzame behandel- en organisatiebesluiten te komen. Dit doen ze door verschillen op te heffen, er ruimte voor te bieden of ze te overbruggen via flexibele verbindingspunten (gewrichten). De successen en de mogelijkheden die gaandeweg aan de orde komen in de dissertatie zijn niet direct een verdienste van de innovatie, maar van haar wisselwerking met het diverse creatieve kennis- en rechtvaardigingswerk van betrokken patiënten en professionals.

Op theoretisch niveau heeft het onderzoek een verdere ontwikkeling van de notie van articulatiewerk opgeleverd. Articulatiewerk, het werk dat nodig is om organisatieprocessen vlot te laten verlopen, heeft niet alleen een temporele en een ruimtelijke dimensie, maar ook een kennis- en morele dimensie. Deze vertonen veel overeenkomsten. Ten eerste, om tot duurzame besluiten te komen is het niet noodzakelijk dat morele of kenniswerelden integreren. Het is vaak voldoende om flexibele schakelpunten te creëren. Ten tweede zijn de processen van kennis en rechtvaardigingswerk niet per se lineair. In de processen waarin duurzame behandel- als organisatiebesluiten tot stand kwamen werden kennis en begrip gewonnen, en voorkeuren gevormd, maar gingen deze ook weer verloren. Ten derde, zulke processen voltrekken zich niet binnen de muren en tijd van een consultatie of een vergadering. Er is sprake van een opeenvolging van momenten van articulatiewerk dat voorafgaat aan het besluit maar dat ook nodig is daarna om het besluit in te bedden in de context waarin het van kracht is. Ten vierde volgt het een bepaalde logica. Kenniswerk volgt epistemische logica's van integratie, verbinding en verdeling; moreel rechtvaardigingswerk volgt centrale waarden als nabijheid en vertrouwen, gelijkheid en burgerschap of efficiëntie.

Als we nu teruggaan naar de drie beloftes van anderhalvelijnszorg dan wordt een aantal praktische implicaties duidelijk. Op basis van de observaties betoogde ik dat kennisintegratie, de eerste belofte, vaak niet nodig is, en dat het streven ernaar wellicht juist tot problemen kan leiden. Kennisintegratie gaat gepaard met kennisreductie en vaak ook met een hiërarchie tussen verschillende vormen van kennis. In plaats daarvan zouden we juist de heterogeniteit van kennis moeten beschermen. De flexibele verbinding van verschillende kennispraktijken via 'gewrichten' maakt dat juist een heterogeen collectief goed in staat is signalen, problemen en fricties op te merken. Ook is het creatiever in het bedenken van oplossingen voor onverwachtse problemen. De ruimte voor diversiteit in kennispraktijken moet dus ook om deze reden beschermd worden, bijvoorbeeld door te waken voor managementtools die kennisreductie bevorderen, of die stimuleren dat medische professionals de problemen van patiënten op eenzelfde uniforme manier bekijken. Ook bij het beoordelen van een kennispraktijk moet er niet steeds van de bevindingen van een andere praktijk uitgegaan worden. Op basis van de analyse van de observaties con- 
cludeerde ik in hoofdstuk vier dat we patiëntgerichtheid moeten begrijpen als een proces, als iets dat opkomt in het creatieve proces van rechtvaardigingswerk dat zich tussen patiënt en medisch specialist voltrekt in de spreekkamer. Als we willen begrijpen hoe patiëntgerichte zorg wordt gedaan, moeten we kijken en reflecteren op hoe professionals en patiënten samen tot duurzame besluiten komen. Exnovatie, in dit geval een proces waarbij professionals samen expliciet maken en reflecteren op hoe ze de situatie aanvoelen, de patiënt inschatten, en ruimte maken voor een diversiteit van concepten van goede zorg is daarvoor bij uitstek geschikt. Het biedt een effectieve manier om professionals te trainen, en de patiëntgerichtheid te verbeteren.

Op dit punt is er dus wel degelijk een interessant leereffect mogelijk: samen stilstaan bij, reflecteren op en dus leren van uitgevoerd kennis- en rechtvaardigingswerk, en daarmee van nieuwe oplossingen, zorgconcepten en overwegingen. De analyses van het vijfde hoofdstuk toonden dat het niet de kostenreductie is die de verplaatsing mogelijk interessant maakt, maar juist het feit dat een dergelijke verandering in de keuzeomgeving routines wegneemt en dus creatieve processen teweegbrengt. Hoe een interventie gemonitord wordt is dus cruciaal. Niet aan de hand van vooraf vastgestelde uitkomstmaten, maar met een open blik. Met uitkomstmaten is er het risico dat de interventie een iteratief proces wordt waarin langzaam de ongewenste elementen eruit gehaald worden en er uiteindelijk een homogene en dus inflexibele zorgpraktijk overblijtt.

Exnovatie en de noties van kennis- en rechtvaardigingswerk, tot slot, bieden de mogelijkheid de empirische ethiek verder te ontwikkelen. Deze noties heffen het vaak gehanteerde verschil op tussen moraal en ethiek. In deze opvatting is de moraal de set aan geaccepteerde en impliciete normen en waarden die het gedrag van mensen in een samenleving structureren; de ethiek de discussie die ontstaat als mensen het niet (meer) met elkaar eens zijn. Op basis van deze tweedeling wordt er meestal een onderscheid gemaakt tussen empirisch en filosofisch onderzoek. In de dissertatie heb ik gepoogd de impliciete moraal van de praktijk te bestuderen vanuit een normatief perspectief die we kennen uit de ethiek. Op de eerste plaats zijn mensen evaluatieve, ofwel normatieve, wezens die zelf al observerend en reflecterend het speelveld navigeren. Het kennis- en rechtvaardigingswerk dat zij uitvoeren is vaak impliciet in hun handelen, maar dat betekent niet dat het zonder spanningen is of dat mensen niet in staat zijn achteraf erop te reflecteren. Juist dit werk volgen en expliciteren maakt het mogelijk om praktijken ethisch te beoordelen zonder tekort te doen aan het rijke en dynamische karakter ervan. 


\section{ABOUT THE AUTHOR}

Willemine Willems (Rotterdam, 1980) is currently affiliated as a postdoctoral researcher and lecturer with the Athena Institute of the Vrije Universiteit, Amsterdam. As a $\mathrm{PhD}$ researcher at the Maastricht University, Willemine worked on an interfaculty project that was a collaboration between the Academic Collaborative Center for Sustainable Care, the School of Business and Economics and the Technology and Society Studies (MUSTS) research group. During the research project, Willemine was part of the department of Philosophy. She was enrolled in the Netherlands Graduate School of Science, Technology, and Modern Culture (WTMC) and became a member of the Ethics \& Politics of Emerging Technology group.

Willemine obtained her MA in Philosophy (cum laude, 2007) and her MSc in Political Sciences (cum laude, 2007) at the University of Amsterdam. As part of the MA-programs, she spent a semester in New York, following courses at the New School University. For her combined Master's thesis about the role of the Truth and Reconciliation Commission in South Africa's transition to democracy she conducted research in Johannesburg at WISER (Wits Institute for Social and Economic Research). As part of her Bachelor program in Philosophy (graduated in 2006, cum laude), she followed a first-year program of natural and social sciences (Betagamma propedeuse).

After graduation, Willemine worked as a researcher at the Rathenau Institute on a project about migration technologies. She was involved in organizing philosophy events. From 2009 onwards, Willemine coordinated, lectured and tutored courses in Philosophy of Science, Ethics and Science and Technology Studies at several departments of the University of Amsterdam, and of several different universities for applied sciences.

Since 2019, Willemine works as a postdoctoral researcher and lecturer at the Athena Institute, Vrije Universiteit Amsterdam. She is part of the Creative Democracy research team, which uses and studies diverse forms of playful participation designed to trigger reflection and dialogue about science and technology in society.

Here, Willemine continuous her interdisciplinary research work, focusing on how Science, Technology and Innovation practices can become more inclusive, democratic and fair. 


\section{CONNECTED AT THE \\ JOINTS}

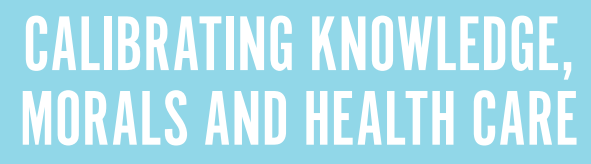

\section{WILLEMNE WILLEMS}

Under the 2013 pioneer policy of the Ministry of Health, a collaboration of national and local care organisations launched a promising innovation in the delivery of specialist health care: Primary Care Plus, a relocation of medical specialist consultations from the hospital to a primary care location. In this book, the author traces the daily work conducted by health care professionals and patients involved in the innovation to attune moral and knowledge differences. By building on these traces of care work, she argues that foregrounding how care delivery and management is done there and then, is an essential part of the fruitful assessment of health care innovation. 